\title{
Visible-Light-Induced Arylthiofluoroalkylations of Unactivated Heteroaromatics and Alkenes
}

\author{
Yeojin Choi, Chunghyeon Yu, Jun Soo Kim, and Eun Jin Cho* \\ Department of Chemistry, Chung-Ang University 84 Heukseok-ro, Dongjak-gu, Seoul 06974, Republic of Korea \\ E-mail: ejcho@cau.ac.kr
}

\section{Supporting Information}

General Considerations

Experimental Details

S-2

Method for DFT Calculation

Preparation of bromoperfluoroalkyl phenyl sulfides (1a, 1b, and 1c)

Experimental procedures for thiofluoroalkylations of heteroaromatics and alkenes

Proposed Mechanisms (Scheme S1 and Scheme S2)

- Optimization of phenylthiodifluoromethylation of 2a (Table S1)

- Optimization of phenylthiotetrafluoroethylation of 2a (Table S2)

- Optimization of phenylthiotetrafluoroethylation of $\mathbf{4 c}$ (Table S3) S-10

- Reactions under oxygen atmosphere (Scheme S3) 


\section{General Considerations}

\section{General Reagent Information}

Anhydrous DMF, DCM, and MeCN were purchased from Sigma-Aldrich chemical company in Sure-Seal bottles and degassed by repeated sonication under light vacuum and replenishing the atmosphere with argon. $f a c$ - $\operatorname{Ir}(\mathrm{ppy})_{3}$ and $\left[\mathrm{Ru}(\mathrm{phen})_{3}\right] \mathrm{Cl}_{2}$ were purchased from Sigma-Aldrich company. All other reagents were purchased from Sigma-Aldrich, Alfa Aesar, Acros Organics, Combi-Blocks or TCI companies. Flash column chromatography was performed using Merck silica gel 60 (70-230 mesh).

\section{General Analytical Information}

The arylthiofluoroalkylated products were characterized by ${ }^{1} \mathrm{H},{ }^{13} \mathrm{C},{ }^{19} \mathrm{~F}$ NMR, and FT-IR spectroscopy. NMR spectra were recorded on a Varian $600 \mathrm{MHz}$ instrument $\left(600 \mathrm{MHz}\right.$ for ${ }^{1} \mathrm{H} \mathrm{NMR}, 151 \mathrm{MHz}$ for ${ }^{13} \mathrm{C}$ NMR, and $564 \mathrm{MHz}$ for ${ }^{19} \mathrm{~F}$ NMR), Varian $300 \mathrm{MHz}$ instrument (300 MHz for crude ${ }^{1} \mathrm{H} \mathrm{NMR}$ ) and a Bruker $400 \mathrm{MHz}$ (377 MHz for ${ }^{19} \mathrm{~F}$ NMR). Copies of ${ }^{1} \mathrm{H},{ }^{13} \mathrm{C}$, and ${ }^{19} \mathrm{~F}$ NMR spectra can be found at the end of the Supporting Information. ${ }^{1} \mathrm{H}$ NMR experiments are reported in units, parts per million (ppm), and were measured relative to residual chloroform $(7.26 \mathrm{ppm})$ in the deuterated solvent. ${ }^{13} \mathrm{C}$ NMR spectra are reported in ppm relative to deuterochloroform $(77.23 \mathrm{ppm})$, and all were obtained with ${ }^{1} \mathrm{H}$ decoupling. ${ }^{19} \mathrm{~F}$ NMR spectra are reported in ppm, and all were taken composite pulse decoupling (CPD) mode. Coupling constants were reported in Hz. FT-IR spectra were recorded on a Nicolet iS 10 ThermoFisher FT-IR spectrometer. Reactions were monitored by GC-MS using the Agilent GC 7890B/5977A inert MSD with Triple-Axis Detector. Mass spectral data of all unknown compounds were obtained from the Korea Basic Science Institute (Daegu) on a Jeol JMS 700 high resolution mass spectrometer.

\section{Experimental Details}

\section{Density functional theory (DFT) calculation of natural charges of radical intermediates}

Density functional theory (DFT) calculations were performed with the Gaussian 09 program package ${ }^{S I}$. Three radical intermediates $\mathbf{1} \mathbf{a}^{\prime}, \mathbf{1} \mathbf{b}^{\prime}$, and $\mathbf{1} \mathbf{c}^{\prime}$, as shown in Fig. 2 of the main text, were optimized by the hybrid B3LYP functional and a $6-31+\mathrm{G}(\mathrm{d})$ basis set. It was shown that the $6-31+\mathrm{G}(\mathrm{d})$ basis set was reasonably good for the description of sulfur-containing compounds ${ }^{S 2}$ and radicals ${ }^{S 3}$. Solvation effects by two relevant solvents, acetonitrile and dichloromethane, were taken into account by the polarization continuum model using the integral equation formalism (IEFPCM) ${ }^{S 4}$. Electron densities of the radical 
intermediates were obtained by the natural bond orbital (NBO) analysis ${ }^{S 5}$ as implemented in the Gaussian 09 program.

In Fig. 2 of the main text, we only presented the natural atomic charges of a radical center carbon, a sulfur atom, and the carbon atoms between them to show the dependence of natural atomic charges on their location relative to the sulfur atom. It is shown that the natural atomic charge of the radical center carbon in $\mathbf{1} \mathbf{a}^{\prime}$ is much less than those in $\mathbf{1} \mathbf{b}^{\prime}$ and $\mathbf{1} \mathbf{c}^{\prime}$, which suggests that the reactivity of the radical intermediate $\mathbf{1} \mathbf{a}^{\prime}$ can be different from those of $\mathbf{1} \mathbf{b}^{\prime}$ and $\mathbf{1} \mathbf{c}^{\prime}$.

The natural atomic charges in different solvent, acetonitrile and dichloromethane, are almost identical only with a negligible difference in the third decimal place, and therefore we presented the values obtained in acetonitrile in Fig. 2. To confirm the validity of the $6-31+G(d)$ basis set, we additionally performed the optimization of the radical intermediate $1 \mathbf{a}^{\prime}$ with a $6-311+\mathrm{G}(3 \mathrm{df}, 2 \mathrm{p})$ and the natural atomic charge of the radical center carbon did not change by more than $3 \%$ from that obtained with the $6-31+G(d)$ $(+0.535$ with the $6-31+\mathrm{G}(\mathrm{d})$ compared with +0.549 with the $6-311+\mathrm{G}(3 \mathrm{df}, 2 \mathrm{p}))$. In addition, we also confirmed that the conformational isomers do not have significant difference in natural atomic charges by performing the optimization of several conformations of $\mathbf{1 b}^{\prime}$ with different dihedral angles defined by four consecutive atoms of C-S-C-C including the radical center carbon. In conclusion, the clear difference between the natural atomic charge of the radical center carbon in $\mathbf{1} \mathbf{a}^{\prime}(+0.535)$ and those in $\mathbf{1 b}^{\prime}(+0.746)$ and $\mathbf{1} \mathbf{c}^{\prime}(+0.757)$ does not depend significantly on the choice of a basis set, a solvent, and a confirmation of the radical intermediates.

\section{Preparation of bromoperfluoroalkyl phenyl sulfide $(\mathbf{1 a}, \mathbf{1 b} \text {, and } 1 \mathrm{c})^{S 6}$}

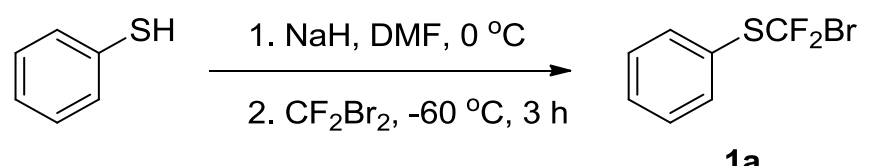

$1 \mathrm{a}$

A round-bottom flask equipped with a magnetic stir bar was flame-dried and charged with argon. Then sodium hydride (60\% dispersion in mineral oil, $1.20 \mathrm{~g}, 30.0 \mathrm{mmol}$ ) and DMF (40 mL) were added, and the flask was cooled down to $0{ }^{\circ} \mathrm{C}$ before the addition of thiophenol $(2.05 \mathrm{~mL}, 20.0 \mathrm{mmol})$. After the slow addition of thiophenol, the reaction mixture was warmed to room temperature for $30 \mathrm{~min}$. The reaction mixture was brought to $-60{ }^{\circ} \mathrm{C}$ and $\mathrm{CF}_{2} \mathrm{Br}_{2}$ reagent $(30.0 \mathrm{mmol})$ was added slowly, and the resulting mixture was maintained at $-60{ }^{\circ} \mathrm{C}$ for $3 \mathrm{~h}$ and at room temperature for $5 \mathrm{~h}$. The reaction progress was checked by thin layer chromatography and gas chromatography. After completion, the reaction was quenched by dropwise addition of water and the aqueous phase was extracted with $\mathrm{Et}_{2} \mathrm{O}$. The separated 
organic layer was washed with brine and dried with $\mathrm{MgSO}_{4}$, filtered, concentrated in vacuo, and purified via silicagel column chromatography using hexanes as eluent to give $\mathrm{PhSCF}_{2} \mathrm{Br}(4.05 \mathrm{~g}, 85 \%)(\mathbf{1 a})$.
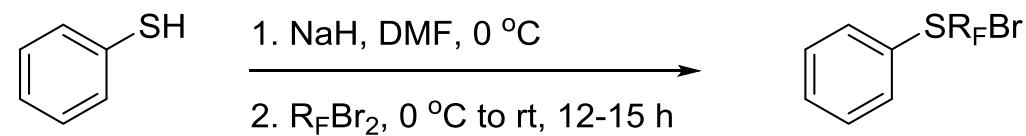

$$
\begin{aligned}
& \text { 1b: } \mathrm{R}_{\mathrm{F}}=-\mathrm{CF}_{2} \mathrm{CF}_{2^{-}} \\
& \text {1c: } \mathrm{R}_{\mathrm{F}}=-\mathrm{CF}_{2} \mathrm{CF}_{2} \mathrm{CF}_{2} \mathrm{CF}_{2^{-}}
\end{aligned}
$$

A round-bottom flask equipped with a magnetic stir bar was flame-dried and charged with thiophenol $(1.03 \mathrm{~mL}, 10.0 \mathrm{mmol})$ and DMF $(50 \mathrm{~mL})$. The mixture was then bubbled with argon for 15 minutes and cooled down to $0{ }^{\circ} \mathrm{C}$. Then sodium hydride $(480 \mathrm{mg}, 12.0 \mathrm{mmol})$ was added slowly to the flask. After 30 minutes, $\mathrm{C}_{2} \mathrm{~F}_{4} \mathrm{Br}_{2}(1.79 \mathrm{~mL}, 15.0 \mathrm{mmol})$ or $\mathrm{C}_{4} \mathrm{~F}_{8} \mathrm{Br}_{2}(2.57 \mathrm{~mL}, 15.0 \mathrm{mmol})$ was added at $0{ }^{\circ} \mathrm{C}$, and the reaction mixture was warmed to room temperature and stirred for 12-15 $\mathrm{h}$. The reaction progress was checked by thin layer chromatography and gas chromatography. After completion, the reaction was quenched by dropwise addition of water and the aqueous phase was extracted with EtOAc. The separated organic layer was washed with brine and dried with $\mathrm{MgSO}_{4}$, filtered, concentrated in vacuo, and purified via silicagel column chromatography using hexanes as eluent to give $\mathrm{PhSC}_{2} \mathrm{~F}_{4} \mathrm{Br}(2.02 \mathrm{~g}, 70 \%)$ (1b) or $\mathrm{PhSC}_{4} \mathrm{~F}_{8} \mathrm{Br}(2.60 \mathrm{~g}, 67 \%)(\mathbf{1 c})$.

\section{Phenylthiodifluoromethylation of heteroaromatics and alkenes}

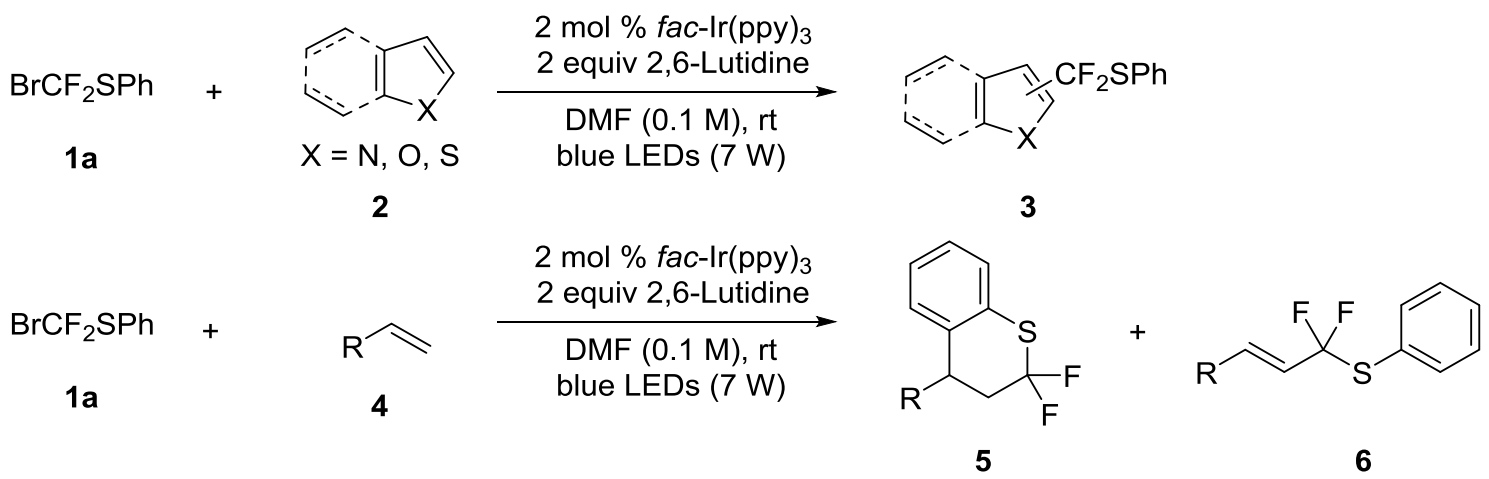

A tube equipped with a magnetic stir bar was flame-dried and charged with argon. $\mathrm{BrCF}_{2} \mathrm{SPh}(0.7 \mathrm{mmol})$, substrate (heteroaromatics 2 or alkene 4: $0.5 \mathrm{mmol}), f a c-\operatorname{Ir}(\mathrm{ppy})_{3}(0.01 \mathrm{mmol}), 2,6$-lutidine $(1 \mathrm{mmol})$, and DMF $(5 \mathrm{~mL})$ were added to the tube. The reaction mixture was then bubbled with argon for 5 minutes and stirred under $7 \mathrm{~W}$ blue LEDs at room temperature for 5 24 h. The reaction progress was checked by thin layer chromatography. The reaction mixture was then diluted with ethyl acetate and washed with brine. The separated organic layer was dried with $\mathrm{MgSO}_{4}$, filtered, concentrated in vacuo, and purified via 
silicagel column chromatography to give the corresponding phenylthiodifluoromethylated product.

Phenylthiotetrafluoroethylation and phenylthiooctafluorobutylation of heteroaromatics

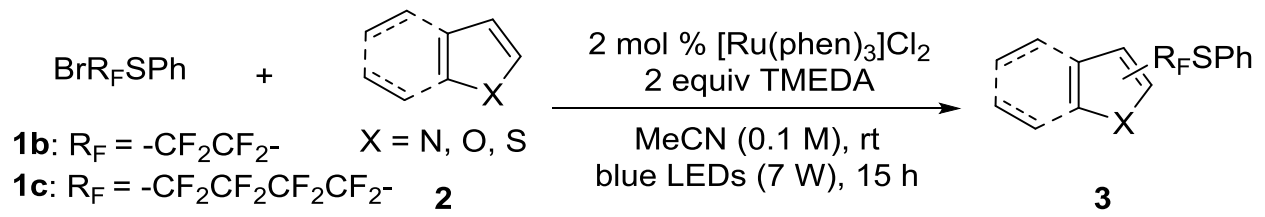

A tube equipped with a magnetic stir bar was flame-dried and charged with argon. $\left[\mathrm{Ru}(\mathrm{phen})_{3}\right] \mathrm{Cl}_{2}(0.01$ $0.15 \mathrm{mmol}), \mathrm{MeCN}(5 \mathrm{~mL})$, TMEDA $(1 \mathrm{mmol})$, and heteroaromatic compound $2(0.5 \mathrm{mmol})$ were added to the tube. After the addition of the phenylthiofluoroalkyl source $(0.7 \mathrm{mmol}), \mathrm{BrCF}_{2} \mathrm{CF}_{2} \mathrm{SPh}$ or $\mathrm{BrCF}_{2} \mathrm{CF}_{2} \mathrm{CF}_{2} \mathrm{CF}_{2} \mathrm{SPh}$, the reaction mixture was bubbled with argon for 3 minutes and stirred under $7 \mathrm{~W}$ blue LEDs at room temperature for $15 \sim 24 \mathrm{~h}$. The reaction progress was checked by thin layer chromatography and gas chromatography. The reaction mixture was then diluted with dichloromethane and washed with water. The separated organic layer was dried with $\mathrm{MgSO}_{4}$, filtered, concentrated in vacuo, and purified via silicagel column chromatography to give the corresponding phenylthiofluoroalkylated heteroaromatic compound.

\section{Phenylthiotetrafluoroethylation and phenylthiooctafluorobutylation of alkenes}

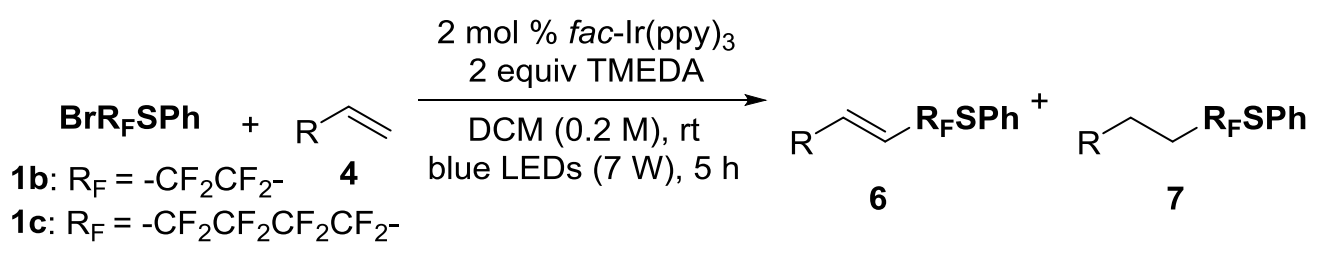

A tube equipped with a magnetic stir bar was flame-dried and charged with argon. $f a c-\operatorname{Ir}(\mathrm{ppy})_{3}(0.01$ mmol), DCM (2.5-3.5 mL), TMEDA (1 mmol), and alkene $4(0.5 \mathrm{mmol})$ were added to the tube. After the addition of the phenylthiofluoroalkyl source (0.7 mmol), $\mathrm{BrCF}_{2} \mathrm{CF}_{2} \mathrm{SPh}$ or $\mathrm{BrCF}_{2} \mathrm{CF}_{2} \mathrm{CF}_{2} \mathrm{CF}_{2} \mathrm{SPh}$, the reaction mixture was bubbled with argon for 10 minutes and stirred under $7 \mathrm{~W}$ blue LEDs at room temperature for 5-6 h. The reaction progress was checked by thin layer chromatography and gas chromatography. The reaction mixture was then diluted with dichloromethane and washed with water. The separated organic layer was dried with $\mathrm{MgSO}_{4}$, filtered, concentrated in vacuo, and purified via silicagel column chromatography to give the corresponding phenylthiofluoroalkylated compound. 


\section{Proposed Mechanism}

A proposed mechanism is shown in Scheme S1 for the phenylthiofluoroalkylation of heteroaromatics using $f a c-\mathrm{Ir}^{\mathrm{III}}(\mathrm{ppy})_{3}$ as the photocatalyst. Photoexcitation of $f a c-\mathrm{Ir}^{\mathrm{III}}(\mathrm{ppy})_{3}$ by visible light produces $\left[\operatorname{Ir}^{\mathrm{IV}} \mathrm{ppy}^{\bullet-}(\mathrm{ppy})_{2}\right]$ through a metal-to-ligand charge-transfer (MLCT) transition. Then the species is oxidatively quenched by one-electron transfer to $\operatorname{Br}\left(\mathrm{CF}_{2}\right)_{n} \mathrm{SPh}(\mathbf{1})$, producing $\left[\operatorname{Ir}^{\mathrm{IV}}(\mathrm{ppy})_{3}\right]^{+}$and the key intermediate $\cdot\left(\mathrm{CF}_{2}\right)_{\mathrm{n}} \mathrm{SPh}\left(\mathbf{1}^{\prime}\right) .^{\mathrm{S} 7}$ The addition of the electron deficient carbon-centered radical $\bullet\left(\mathrm{CF}_{2}\right)_{\mathrm{n}} \mathrm{SPh}\left(\mathbf{1}^{\prime}\right)$ to a heteroaromatic compound (2) generates the phenylthiofluoroalkylated radical species $\mathbf{2}^{\prime}$ Then, a one-electron transfer from $\mathbf{2}^{\prime}$ to $\left[\operatorname{Ir}^{\mathrm{IV}}(\mathrm{ppy})_{3}\right]^{+}$ regenerates $\operatorname{Ir}^{\mathrm{III}}(\mathrm{ppy})_{3}$ and forms a carbocation intermediate $\mathbf{2}^{\prime \prime}$. Subsequent deprotonation by a base completes the reaction providing the desired product 3 .

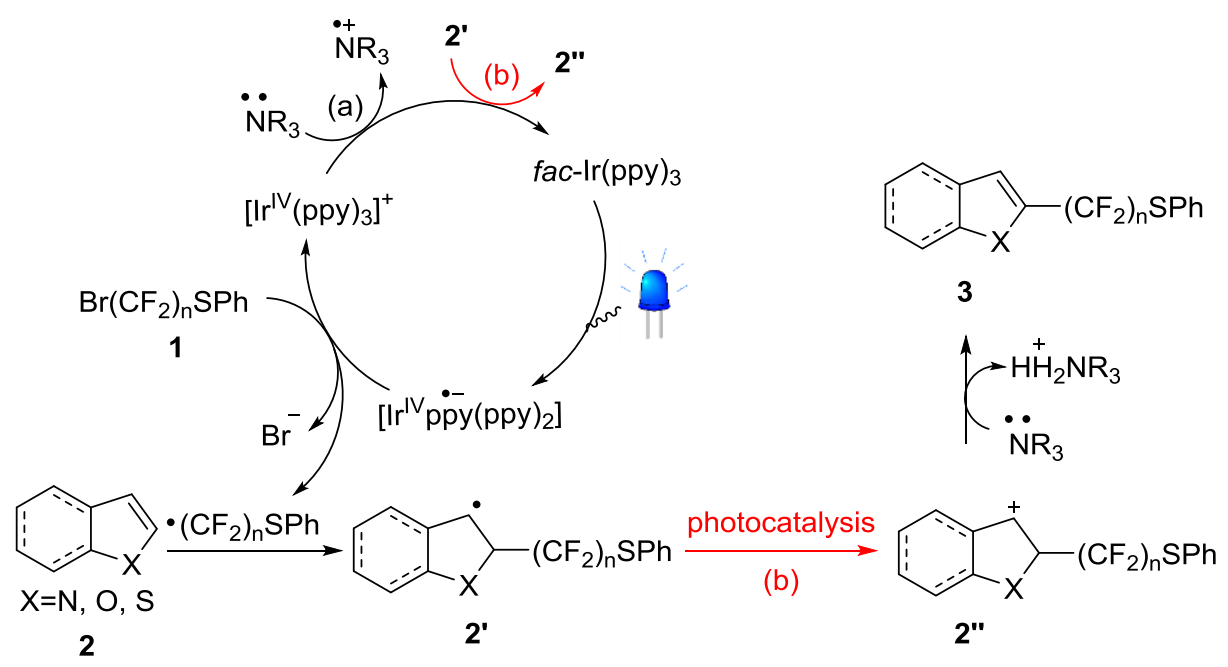

Scheme S1. Proposed mechanism for phenylthiofluoroalkylation of heteroaromatics.

The proposed mechanism for the phenylthiofluoroalkylations of alkenes is illustrated in Scheme S2. Similary to the reaction of heteroaromatics, photoexcitation of $f a c-\mathrm{Ir}^{\mathrm{III}}(\mathrm{ppy})_{3}$ produces MLCT excited state $\left[\operatorname{Ir}^{\mathrm{IV}} \mathrm{ppy}^{\bullet-}(\mathrm{ppy})_{2}\right]$, which is oxidatively quenched by single electron transfer to $\operatorname{Br}\left(\mathrm{CF}_{2}\right)_{\mathrm{n}} \mathrm{SPh}(\mathbf{1})$ to produce the key intermediate $\cdot\left(\mathrm{CF}_{2}\right)_{\mathrm{n}} \mathrm{SPh}\left(\mathbf{1}^{\prime}\right)$. Addition of $\bullet\left(\mathrm{CF}_{2}\right)_{\mathrm{n}} \mathrm{SPh}\left(\mathbf{1}^{\prime}\right)$ to the alkene (4) generates the phenylthiofluoroalkylated radical species 4', which participates in several reactions depending on the phenylthiofluroalkyl source, substituent pattern of alkene, and base. First, in the reaction of aliphatic alkenes using $\mathrm{BrCF}_{2} \mathrm{SPh}(\mathbf{1 a})$, the intermediate $\mathbf{4}^{\prime}$ can preferentially undergo an aromatic substitution with thethered phenyl ring to give 5'. Its subsequent photocatalytic oxidation to $\mathbf{5}^{\prime \prime}$ follwed by deprotonation provides 5 . Phenylthiofluoromethylated alkenes 6 are generated as the major product in the reactions of aromatic alkenes through photocatalytic oxidation of $\mathbf{4}^{\prime}$ to $\mathbf{4}^{\prime \prime}$ followed by its deprotonation. On 
the other hand, the reaction of aliphatic alkenes using $\mathbf{1 b}^{\prime}$ or $\mathbf{1} \mathbf{c}^{\prime}$ in the presence of TMEDA provides hydrophenylthiofluoroalkylated products 7 as the major product. The $\alpha$-hydrogen atom of $\left[\mathrm{NR}_{3}\right]^{++}$intermediate formed from the tertiary amine by photocatalysis could serve as a hydrogen atom donor.

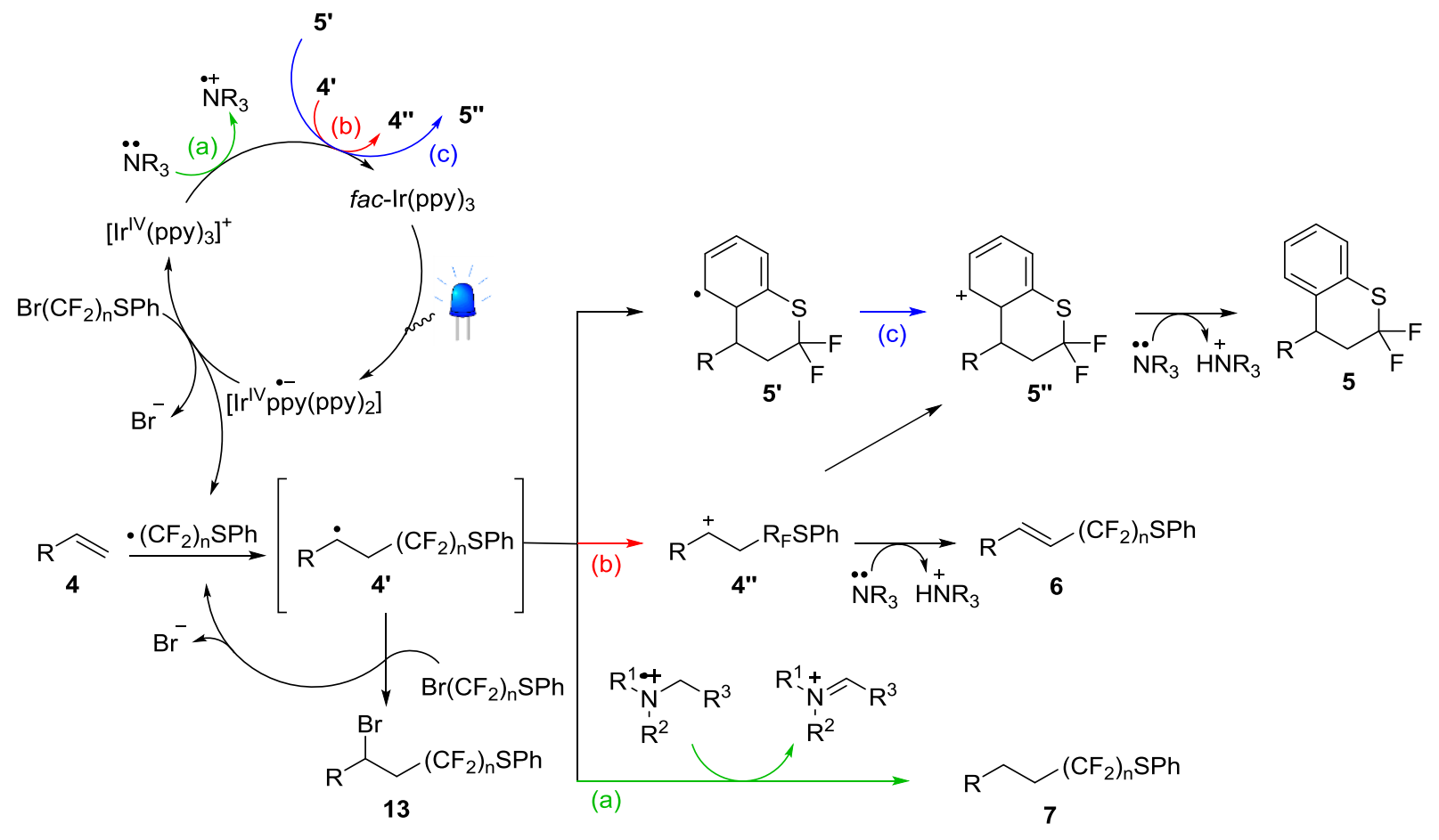

Scheme S2. Proposed mechanism for phenylthiofluoroalkylation of alkenes. 


\section{Additional Experimental Results}

Table S1. Optimization of phenylthiodifluoromethylation of $\mathbf{2} \mathbf{a}^{a}$

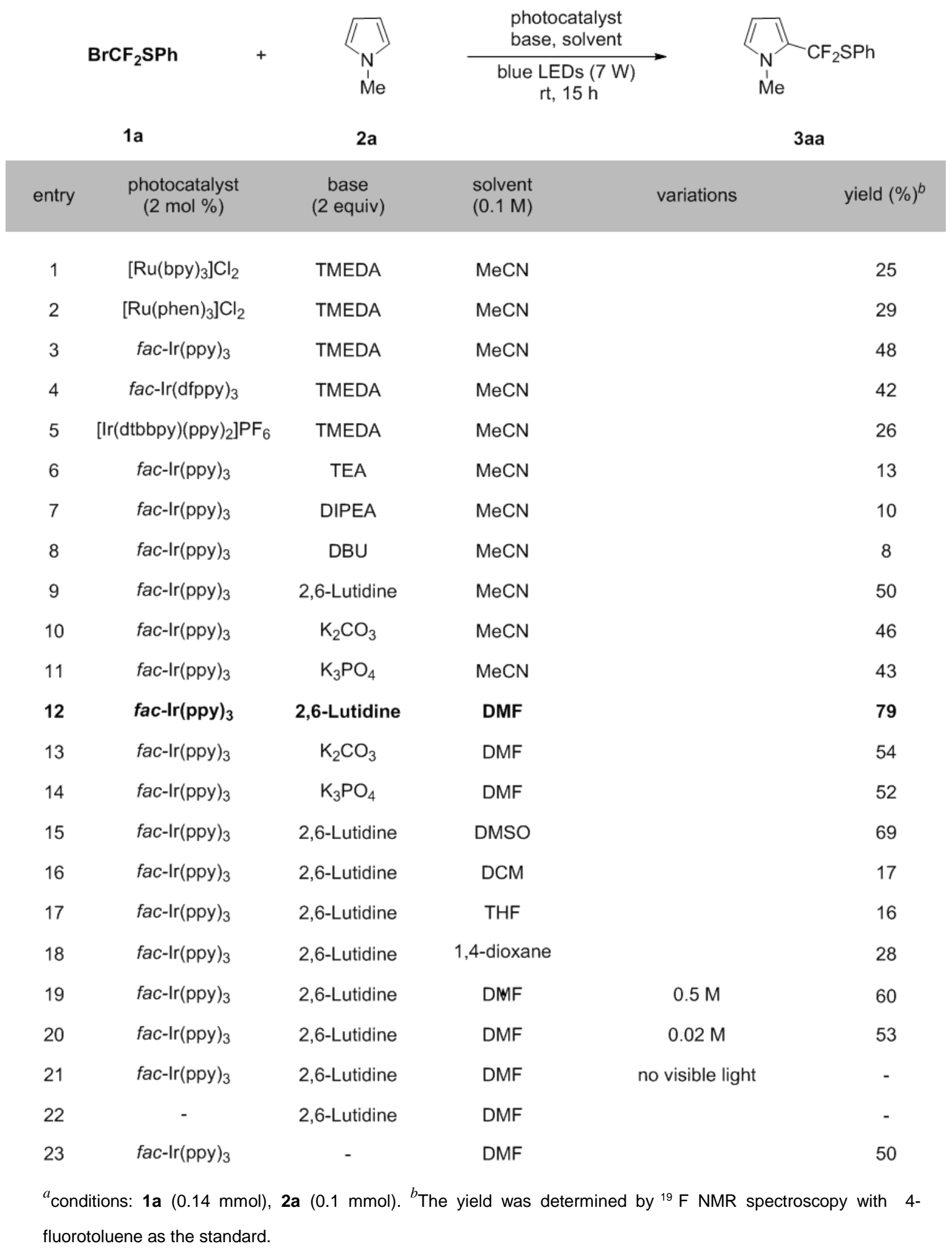


Table S2. Optimization of phenylthiotetrafluoroethylation of $\mathbf{2} \mathbf{a}^{a}$

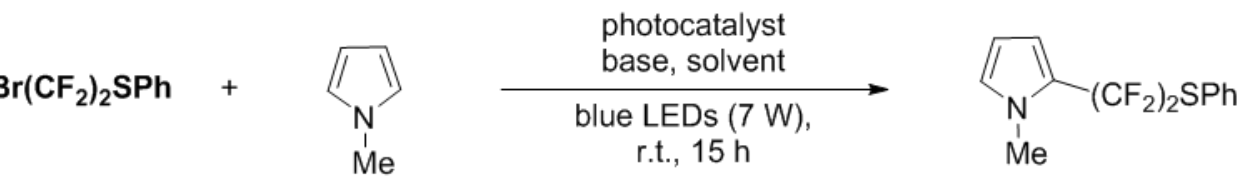

1b

2a

3ba

\begin{tabular}{|c|c|c|c|c|c|}
\hline Entry & $\begin{array}{l}\text { Photocatalyst } \\
(2 \mathrm{~mol} \%)\end{array}$ & $\begin{array}{c}\text { Base } \\
\text { (2 equiv.) }\end{array}$ & $\begin{array}{l}\text { Solvent } \\
(0.1 \mathrm{M})\end{array}$ & Variations & $\begin{array}{c}\text { Yield }(\%)^{b} \\
\text { 3ba }\end{array}$ \\
\hline 1 & {$\left[\mathrm{Ru}(\text { phen })_{3}\right] \mathrm{Cl}_{2}$} & TMEDA & $\mathrm{MeCN}$ & & 81 \\
\hline 2 & {$\left[\mathrm{Ru}(\mathrm{bpy})_{3}\right] \mathrm{Cl}_{2}$} & TMEDA & $\mathrm{MeCN}$ & & 75 \\
\hline 3 & $f a c-\operatorname{Ir}(\mathrm{ppy})_{3}$ & TMEDA & $\mathrm{MeCN}$ & & 81 \\
\hline 4 & fac-Ir(dfppy $)_{3}$ & TMEDA & $\mathrm{MeCN}$ & & - \\
\hline 5 & {$\left[\mathrm{Ir}(\mathrm{dtbbpy})(\mathrm{ppy})_{2}\right] \mathrm{PF}_{6}$} & TMEDA & $\mathrm{MeCN}$ & & 67 \\
\hline 6 & $f a c-\operatorname{Ir}(\mathrm{ppy})_{3}$ & 2,6-Lutidine & DMF & & - \\
\hline 7 & {$\left[\mathrm{Ru}(\text { phen })_{3}\right] \mathrm{Cl}_{2}$} & 2,6-Lutidine & $\mathrm{MeCN}$ & & - \\
\hline 8 & {$\left[\mathrm{Ru}(\text { phen })_{3}\right] \mathrm{Cl}_{2}$} & DBU & $\mathrm{MeCN}$ & & 57 \\
\hline 9 & {$\left[\mathrm{Ru}(\text { phen })_{3}\right] \mathrm{Cl}_{2}$} & DIPEA & $\mathrm{MeCN}$ & & 45 \\
\hline 10 & {$\left[\mathrm{Ru}(\text { phen })_{3}\right] \mathrm{Cl}_{2}$} & $\mathrm{~K}_{2} \mathrm{CO}_{3}$ & $\mathrm{MeCN}$ & & - \\
\hline 11 & {$\left[\mathrm{Ru}(\text { phen })_{3}\right] \mathrm{Cl}_{2}$} & TMEDA & DMF & & 70 \\
\hline 12 & {$\left[\mathrm{Ru}(\text { phen })_{3}\right] \mathrm{Cl}_{2}$} & TMEDA & DMSO & & 68 \\
\hline 13 & {$\left[\mathrm{Ru}(\text { phen })_{3}\right] \mathrm{Cl}_{2}$} & TMEDA & DCM & & 39 \\
\hline 14 & {$\left[\mathrm{Ru}(\text { phen })_{3}\right] \mathrm{Cl}_{2}$} & TMEDA & $\mathrm{MeOH}$ & & - \\
\hline 15 & {$\left[\mathrm{Ru}(\text { phen })_{3}\right] \mathrm{Cl}_{2}$} & TMEDA & $\mathrm{MeCN}$ & $0.5 \mathrm{M}$ & - \\
\hline 16 & {$\left[\mathrm{Ru}(\text { phen })_{3}\right] \mathrm{Cl}_{2}$} & TMEDA & $\mathrm{MeCN}$ & $0.2 \mathrm{M}$ & 68 \\
\hline 17 & {$\left[\mathrm{Ru}(\text { phen })_{3}\right] \mathrm{Cl}_{2}$} & TMEDA & $\mathrm{MeCN}$ & $0.05 \mathrm{M}$ & 46 \\
\hline 18 & - & TMEDA & $\mathrm{MeCN}$ & & - \\
\hline 19 & {$\left[\mathrm{Ru}(\text { phen })_{3}\right] \mathrm{Cl}_{2}$} & - & $\mathrm{MeCN}$ & & 47 \\
\hline 20 & {$\left[\mathrm{Ru}(\text { phen })_{3}\right] \mathrm{Cl}_{2}$} & TMEDA & $\mathrm{MeCN}$ & no visible light & trace \\
\hline 21 & {$\left[\mathrm{Ru}(\text { phen })_{3}\right] \mathrm{Cl}_{2}$} & TMEDA & $\mathrm{MeCN}$ & $3 \mathrm{~mol} \%\left[\mathrm{Ru}(\text { phen })_{3}\right] \mathrm{Cl}_{2}$ & 84 \\
\hline
\end{tabular}

${ }^{a}$ Reaction conditions: 1b $(0.14 \mathrm{mmol}), \mathbf{2 a}(0.1 \mathrm{mmol}) .{ }^{b}$ The yield was determined by gas chromatography and ${ }^{19} \mathrm{~F}$ NMR spectroscopy with internal standards, dodecane and 4-fluorotoluene, respectively. 
Table S3. Optimization of phenylthiotetrafluoroethylation of $4 c^{a}$

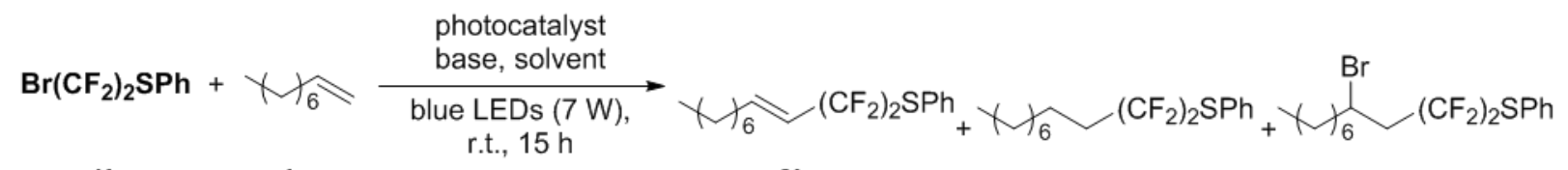

1b

$4 \mathrm{c}$ 6bc

$7 \mathrm{bc}$

\begin{tabular}{|c|c|c|c|c|c|c|c|}
\hline \multirow{2}{*}{ Entry } & \multirow{2}{*}{$\begin{array}{l}\text { Photocatalyst } \\
(2 \mathrm{~mol} \%)\end{array}$} & \multirow{2}{*}{$\begin{array}{c}\text { Base } \\
\text { (2 equiv.) }\end{array}$} & \multirow{2}{*}{$\begin{array}{l}\text { Solvent } \\
(0.2 \mathrm{M})\end{array}$} & \multirow{2}{*}{ Variations } & \multicolumn{3}{|c|}{ Yield $(\%)^{b}$} \\
\hline & & & & & $6 b c$ & $7 \mathrm{bc}$ & $13 b c$ \\
\hline 1 & {$\left[\mathrm{Ru}(\text { phen })_{3}\right] \mathrm{Cl}_{2}$} & TMEDA & DCM & & - & - & - \\
\hline 2 & {$\left[\mathrm{Ru}(\mathrm{bpy})_{3}\right] \mathrm{Cl}_{2}$} & TMEDA & DCM & & - & - & - \\
\hline 3 & $f a c-\operatorname{Ir}(p p y)_{3}$ & TMEDA & DCM & & 12 & 75 & - \\
\hline 4 & $f a c-\operatorname{Ir}(\mathrm{dfppy})_{3}$ & TMEDA & DCM & & 8 & 78 & - \\
\hline 5 & {$\left[\mathrm{Ir}(\mathrm{dtbbpy})(\mathrm{ppy})_{2}\right] \mathrm{PF}_{6}$} & TMEDA & DCM & & 11 & 76 & - \\
\hline 6 & $f a c-\operatorname{Ir}(\mathrm{ppy})_{3}$ & 2,6-Lutidine & DCM & & - & - & 17 \\
\hline 7 & $f a c-\operatorname{Ir}(\mathrm{ppy})_{3}$ & DBU & DCM & & 6 & 52 & - \\
\hline 8 & $f a c-\operatorname{Ir}(\mathrm{ppy})_{3}$ & DIPEA & DCM & & trace & 7 & - \\
\hline 9 & $f a c-\operatorname{Ir}(\mathrm{ppy})_{3}$ & $\mathrm{~K}_{2} \mathrm{CO}_{3}$ & DCM & & - & - & 32 \\
\hline 10 & $f a c-\operatorname{Ir}(\mathrm{ppy})_{3}$ & TEA & DCM & & 3 & 21 & - \\
\hline 11 & $f a c-\operatorname{Ir}(\mathrm{ppy})_{3}$ & TMEDA & $\mathrm{MeCN}$ & & 17 & 40 & - \\
\hline 12 & $f a c-\operatorname{Ir}(\mathrm{ppy})_{3}$ & TMEDA & DMF & & 7 & 64 & - \\
\hline 13 & $f a c-\operatorname{Ir}(\mathrm{ppy})_{3}$ & TMEDA & DMSO & & 8 & 33 & - \\
\hline 14 & $f a c-\operatorname{Ir}(\mathrm{ppy})_{3}$ & TMEDA & $\mathrm{MeOH}$ & & 2 & 11 & - \\
\hline 15 & $f a c-\operatorname{Ir}(\mathrm{ppy})_{3}$ & TMEDA & DCM & $0.5 \mathrm{M}$ & 8 & 37 & - \\
\hline 16 & $f a c-\operatorname{Ir}(\mathrm{ppy})_{3}$ & TMEDA & DCM & $0.1 \mathrm{M}$ & 12 & 53 & - \\
\hline 17 & $f a c-\operatorname{Ir}(\mathrm{ppy})_{3}$ & TMEDA & DCM & $0.05 \mathrm{M}$ & 5 & 36 & - \\
\hline 18 & - & TMEDA & DCM & & - & - & - \\
\hline 19 & $f a c-\operatorname{Ir}(\mathrm{ppy})_{3}$ & - & DCM & & trace & trace & trace \\
\hline 20 & $f a c-\operatorname{Ir}(\mathrm{ppy})_{3}$ & TMEDA & DCM & no visible light & - & - & - \\
\hline 21 & $f a c-\operatorname{Ir}(\mathrm{ppy})_{3}$ & TMEDA & DCM & not degassed & 7 & 46 & - \\
\hline
\end{tabular}

${ }^{a}$ Reaction conditions: $\mathbf{1 b}(0.14 \mathrm{mmol}), \mathbf{4 e}(0.1 \mathrm{mmol}) .{ }^{b}$ The yield was determined by gas chromatography and ${ }^{19} \mathrm{~F}$ NMR spectroscopy with internal standards, dodecane and 4-fluorotoluene, respectively. 


\section{Reactions under oxygen atmosphere}

In the reactions of alkenes, we found another reactivity difference between reactions using $\mathbf{1 a}$ and $\mathbf{1 b}$ when conducted in an oxygen atmosphere. In the presence of molecular oxygen, the reactivity of the reaction with $\mathbf{4 b}$ using $1 \mathbf{a}$ significantly decreased, probably due to the competitive photocatalytic pathway involving oxygen [Scheme S3 (1)]. On the other hand, the reaction of $\mathbf{4 b}$ with $\mathbf{1 b}$ proceeded well with no deterioration of yield to give hydro-phenylthiotetrafluoroethylated product, and further oxidation of sulfur occurred to provide sulfoxide S1 as the final product [Scheme S3 (2)]. It is likely that the delocalization of lone pair of electrons on the sulfur in radical intermediate $\mathbf{1} \mathbf{a}^{\prime}$ through the carbon radical center might prevent oxidation of sulfur whereas the sulfur in $\mathbf{1} \mathbf{b}^{\prime}$, located further from the radical center, can undergo further oxidation in the presence of oxygen.

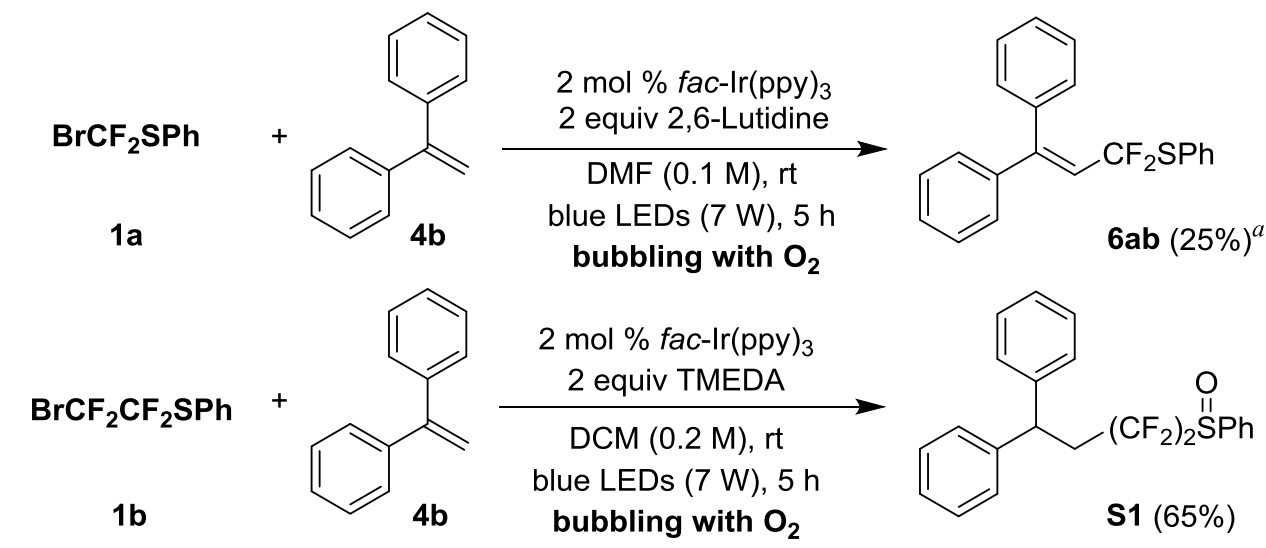

Scheme S3. Reactions of $\mathbf{4 b}$ under oxygen atmosphere: ${ }^{a}$ The unreacted $\mathbf{4 b}$ was recovered.

\section{Analytic Data for Arylthiofluoroalkylated Compounds}

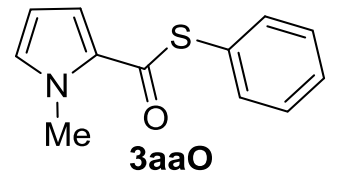

from

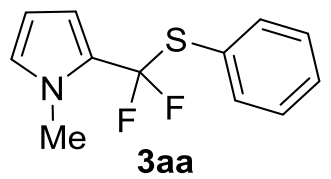

3aa: ${ }^{1} \mathrm{H}$ NMR of crude sample (300 MHz, DMSO) $\delta 7.62-7.43(\mathrm{~m}, 5 \mathrm{H}), 6.94(\mathrm{dd}, J=2.4,2.2 \mathrm{~Hz}, 1 \mathrm{H})$, $6.32-6.30(\mathrm{~m}, 1 \mathrm{H}), 5.98(\mathrm{dd}, J=3.8,2.4 \mathrm{~Hz}, 1 \mathrm{H}), 3.75(\mathrm{~s}, 3 \mathrm{H}) ;{ }^{19} \mathbf{F}$ NMR $\left(377 \mathbf{~ M H z}, \mathbf{C D C l}_{3}\right) \delta-65.72$ 3aaO: light yellow oil (83.5 mg, 77\%); ${ }^{1} \mathbf{H}$ NMR (600 MHz, CDCl $) \delta 7.51$ (d, $\left.J=4.5 \mathrm{~Hz}, 2 \mathrm{H}\right), 7.46-$ $7.42(\mathrm{~m}, 3 \mathrm{H}), 7.23(\mathrm{~d}, J=3.8 \mathrm{~Hz}, 1 \mathrm{H}), 6.85(\mathrm{~s}, 1 \mathrm{H}), 6.18(\mathrm{~s}, 1 \mathrm{H}), 3.87(\mathrm{~s}, 3 \mathrm{H}) ;{ }^{13} \mathbf{C}$ NMR (151 MHz, 
$\left.\mathbf{C D C l}_{3}\right) \delta=180.25,135.65,131.15,129.48,129.30,128.71,127.99,118.98,108.91,37.41$; IR (neat): $v_{\max }=1653,1398,1371,1202,845,744 \mathrm{~cm}^{-1} ; \boldsymbol{R}_{f} 0.50($ hex/EtOAc, $1 / 4)$.

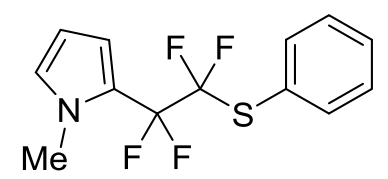

3ba: light yellow oil (94.0 mg, 65\%); ${ }^{1} \mathbf{H}$ NMR (600 MHz, $\left.\mathbf{C D C l}_{3}\right) \delta 7.64(\mathrm{~d}, J$ $=7.7 \mathrm{~Hz}, 2 \mathrm{H}), 7.46(\mathrm{t}, J=7.2 \mathrm{~Hz}, 1 \mathrm{H}), 7.39(\mathrm{dd}, J=7.7,7.2 \mathrm{~Hz}, 2 \mathrm{H}), 6.72-$ $6.69(\mathrm{~m}, 1 \mathrm{H}), 6.62-6.58(\mathrm{~m}, 1 \mathrm{H}), 6.15-6.12(\mathrm{~m}, 1 \mathrm{H}), 3.72(\mathrm{~s}, 3 \mathrm{H}) ;{ }^{13} \mathbf{C} \mathbf{N M R}$ (151 MHz, $\left.\mathbf{C D C l}_{3}\right) \delta 137.24,130.55,129.34,127.61,124.67,120.40\left(\mathrm{t}, J_{\mathrm{C}-\mathrm{F}}=30.2 \mathrm{~Hz}\right), 114.18,107.72$, 36.06 (carbon peaks of $-\mathrm{C}_{2} \mathrm{~F}_{4}$ - are omitted due to complicated C-F splitting); ${ }^{19} \mathbf{F}$ NMR (377 MHz, $\left.\mathbf{C D C l}_{3}\right) \delta$-86.90, -102.18; IR (neat): $v_{\max }=2925,1542,1318,1111,1093,1080,1061,906,730 \mathrm{~cm}^{-1}$; HRMS m/z (EI) calc. for $\mathrm{C}_{13} \mathrm{H}_{11} \mathrm{~F}_{4} \mathrm{NS}$ [M+] 289.0548, found 289.0545; $\boldsymbol{R}_{\boldsymbol{f}} 0.57$ (hex/EtOAc, 8/1).

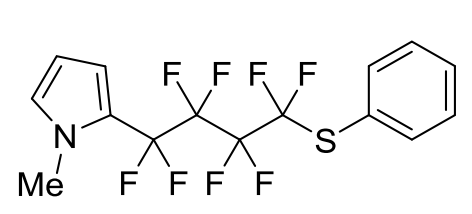

3ca: light yellow oil (116.7 mg, 60\%); ${ }^{1} \mathbf{H}$ NMR (600 $\left.\mathbf{M H z}, \mathbf{C D C l}_{3}\right) \delta$ $7.69(\mathrm{~d}, J=7.4 \mathrm{~Hz}, 2 \mathrm{H}), 7.51(\mathrm{t}, J=7.7 \mathrm{~Hz}, 1 \mathrm{H}), 7.43(\mathrm{dd}, J=7.7 \mathrm{~Hz}, 7.4$ $\mathrm{Hz}, 2 \mathrm{H}), 6.74(\mathrm{~d}, J=3.8 \mathrm{~Hz}, 1 \mathrm{H}), 6.59(\mathrm{~d}, J=3.0 \mathrm{~Hz}, 1 \mathrm{H}), 6.17(\mathrm{dd}, J=$ 3.8, 3.0 Hz, 1H), 3.72 (s, 3H); ${ }^{13} \mathbf{C}$ NMR (151 MHz, CDCl $) \delta$ 137.62, $131.05,129.52,128.05,123.49,119.64\left(\mathrm{t}, J_{\mathrm{C}-\mathrm{F}}=30.1 \mathrm{~Hz}\right.$ ), 114.49, 107.98, 35.99 (carbon peaks of $-\mathrm{C}_{4} \mathrm{~F}_{8}-$ are omitted due to complicated C-F splitting); ${ }^{19} \mathbf{F}$ NMR (564 $\left.\mathbf{~ M H z , ~} \mathbf{C D C l}_{3}\right) \delta$-86.54, -104.46, -118.81, 120.15; IR (neat): $v_{\max }=2963,1542,1434,1171,1117,725 \mathrm{~cm}^{-1} ; \mathbf{H R M S ~ m} / \mathrm{z}$ (EI) calc. for $\mathrm{C}_{15} \mathrm{H}_{11} \mathrm{~F}_{8} \mathrm{NS}$ $[\mathrm{M}+]$ 389.0484, found 389.0482; $\boldsymbol{R}_{f} 0.59$ (hex/EtOAc, 8/1).

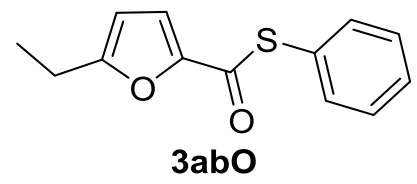

from

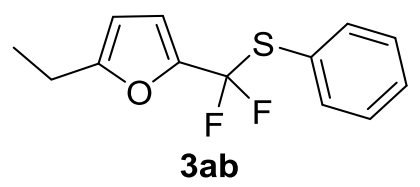

3ab: ${ }^{1} \mathrm{H}$ NMR of crude sample (300 MHz, DMSO) $\delta 7.38-7.56(\mathrm{~m}, 5 \mathrm{H}), 6.70(\mathrm{~d}, J=3.4 \mathrm{~Hz}, 1 \mathrm{H}), 6.17$ $(\mathrm{d}, J=3.4 \mathrm{~Hz}, 1 \mathrm{H}), 2.63(\mathrm{q}, J=7.4 \mathrm{~Hz}, 2 \mathrm{H}), 1.14(\mathrm{t}, J=7.4 \mathrm{~Hz}, 3 \mathrm{H}) ;{ }^{19} \mathbf{F} \mathbf{N M R}\left(377 \mathbf{M H z}, \mathbf{C D C l}_{3}\right) \delta$ 69.14

3abO: colorless oil (87.9 mg, 76\%); ${ }^{1} \mathbf{H}$ NMR (600 $\left.\mathbf{M H z}, \mathbf{C D C l}_{3}\right) \delta 7.52-7.49$ (m, 2H), $7.45-7.43(\mathrm{~m}$, $3 \mathrm{H}), 7.20(\mathrm{~d}, J=3.5 \mathrm{~Hz}, 1 \mathrm{H}), 6.20(\mathrm{~d}, J=3.5 \mathrm{~Hz}, 1 \mathrm{H}), 2.77(\mathrm{q}, J=7.6 \mathrm{~Hz}, 2 \mathrm{H}), 1.30(\mathrm{~d}, J=7.6 \mathrm{~Hz}, 3 \mathrm{H})$; ${ }^{13} \mathbf{C}$ NMR (151 MHz, $\left.\mathbf{C D C l}_{3}\right) \delta=178.17,163.50,148.97,135.35,129.65,129.36,126.80,118.08$, 107.79, 22.00, 11.99; IR (neat): $v_{\max }=2975,1656,1509,1257,1016,977,843,746 \mathrm{~cm}^{-1} ; \boldsymbol{R}_{f} 0.57$ (hex/EtOAc, 4/1). 


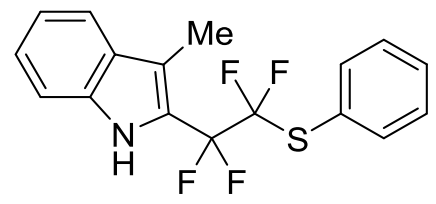

3bc: light yellow oil (118.7 mg, 70\%); ${ }^{1} \mathbf{H}$ NMR (600 $\left.\mathbf{M H z}, \mathbf{C D C l}_{3}\right) \delta 8.15$ $(\mathrm{s}, 1 \mathrm{H}), 7.65(\mathrm{~d}, J=8.0 \mathrm{~Hz}, 1 \mathrm{H}), 7.61(\mathrm{~d}, J=7.6 \mathrm{~Hz}, 2 \mathrm{H}), 7.44(\mathrm{dd}, J=7.6,7.5 \mathrm{~Hz}$ $1 \mathrm{H}), 7.39-7.34(\mathrm{~m}, 3 \mathrm{H}), 7.32(\mathrm{t}, J=7.5 \mathrm{~Hz}, 1 \mathrm{H}), 7.19(\mathrm{dd}, J=7.6,7.5 \mathrm{~Hz}, 1 \mathrm{H})$, $2.45(\mathrm{~s}, 3 \mathrm{H}) ;{ }^{13} \mathbf{C}$ NMR $\left(\mathbf{1 5 1} \mathbf{~ M H z}, \mathbf{C D C l}_{3}\right) \delta 137.14,135.94,130.61,129.34$, $128.62,124.62,124.23,121.27,120.27,120.09,115.81,111.5781,8.99$ (carbon peaks of $-\mathrm{C}_{4} \mathrm{~F}_{8}-$ are omitted due to complicated C-F splitting); ${ }^{19} \mathbf{F}$ NMR (377 $\left.\mathbf{M H z}, \mathbf{C D C l}_{3}\right) \delta$-86.87, -105.65; IR (neat): $v_{\max }=2926,1607,1487,1312,1094,747 \mathrm{~cm}^{-1}$; HRMS m/z (EI) calc. for $\mathrm{C}_{17} \mathrm{H}_{13} \mathrm{~F}_{4} \mathrm{NS}$ [M+] 339.0705, found $339.0709 ; \boldsymbol{R}_{f} 0.42$ (hex/EtOAc, 8/1).

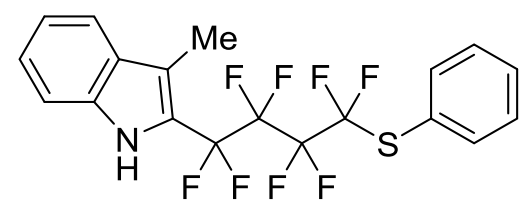

3cc: light yellow oil (171.3 mg, 78\%); ${ }^{1} \mathbf{H}$ NMR (600 $\left.\mathbf{M H z}, \mathbf{C D C l}_{3}\right)$ $\delta 7.70-7.65(\mathrm{~m}, 3 \mathrm{H}), 7.52-7.47(\mathrm{~m}, 2 \mathrm{H}), 7.45-7.40(\mathrm{~m}, 4 \mathrm{H}), 2.45$ $(\mathrm{s}, 3 \mathrm{H}) ;{ }^{13} \mathrm{C}$ NMR (151 MHz, $\left.\mathbf{C D C l}_{3}\right) \delta$ 137.62, 136.02, 131.09, $129.54,128.56,124.94,123.3625,120.44,120.33\left(\mathrm{t}, J_{\mathrm{C}-\mathrm{F}}=28.7 \mathrm{~Hz}\right)$, 120.22, 116.53, 111.66, 8.82 (carbon peaks of $-\mathrm{C}_{4} \mathrm{~F}_{8}-$ are omitted due to complicated C-F splitting); ${ }^{19} \mathbf{F}$ NMR (564 MHz, CDCl $\left.\mathbf{~}_{3}\right) \delta$-86.56, -108.59, -119.02, -121.45; IR (neat): $v_{\max }=3063,2927,1589,1316$, 1242, 1175, 1117, $745 \mathrm{~cm}^{-1}$; HRMS m/z (EI) calc. for $\mathrm{C}_{19} \mathrm{H}_{13} \mathrm{~F}_{8} \mathrm{NS}$ [M+] 439.0641, found 439.0643; $\boldsymbol{R}_{\boldsymbol{f}}$ 0.47 (hex/EtOAc, 8/1).

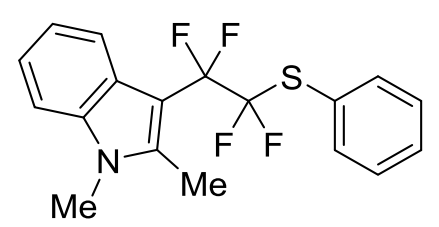

3bd: White solid (77.7 mg, 44\%); ${ }^{1} \mathbf{H}$ NMR (600 $\left.\mathbf{M H z}, \mathbf{C D C l}_{3}\right) \delta 7.73(\mathrm{~d}, J$ $=7.8 \mathrm{~Hz}, 1 \mathrm{H}), 7.60(\mathrm{~d}, J=7.6 \mathrm{~Hz}, 2 \mathrm{H}), 7.41(\mathrm{t}, J=7.8 \mathrm{~Hz}, 1 \mathrm{H}), 7.34(\mathrm{dd}, J$ $=7.8,7.6 \mathrm{~Hz}, 2 \mathrm{H}), 7.28(\mathrm{~d}, J=8.0 \mathrm{~Hz}, 1 \mathrm{H}), 7.22(\mathrm{dd}, J=7.8,7.5 \mathrm{~Hz}, 1 \mathrm{H})$, $7.16(\mathrm{dd}, J=8.0,7.5 \mathrm{~Hz}, 1 \mathrm{H}), 3.66(\mathrm{~s}, 3 \mathrm{H}), 2.50(\mathrm{~s}, 3 \mathrm{H}) ;{ }^{13} \mathrm{C}$ NMR (151 MHz, $\left.\mathbf{C D C l}_{3}\right) \delta 137.16,136.97,136.65,130.22,129.18,125.97,125.11$, $121.95,120.98,120.31,119.81,109.25,29.76,11.64$ (carbon peaks of $-\mathrm{C}_{2} \mathrm{~F}_{4}-$ are omitted due to complicated C-F splitting); ${ }^{19} \mathbf{F}$ NMR (377 $\mathbf{M H z}, \mathbf{C D C l}_{3}$ ) $\delta$-87.11, -101.31; IR (neat): $v_{\max }=1559,1475$, 1093, 1068, $743 \mathrm{~cm}^{-1}$; HRMS m/z (EI) calc. for $\mathrm{C}_{18} \mathrm{H}_{15} \mathrm{~F}_{4} \mathrm{NS}[\mathrm{M}+]$ 353.0861, found 353.0862; $\boldsymbol{R}_{\boldsymbol{f}} 0.46$ (hex/EtOAc, 4/1).

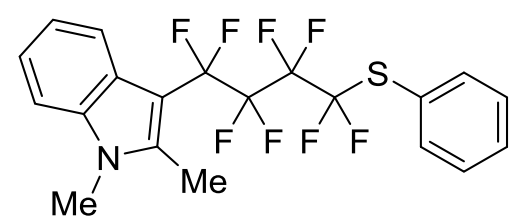

3cd: White solid (151.8 mg, 67\%); ${ }^{1} \mathbf{H}$ NMR (600 $\left.\mathbf{M H z}, \mathbf{C D C l}_{3}\right) \delta$ $7.71-7.63(\mathrm{~m}, 3 \mathrm{H}), 7.48(\mathrm{t}, J=7.4 \mathrm{~Hz}, 1 \mathrm{H}), 7.41(\mathrm{dd}, J=7.5,7.4 \mathrm{~Hz}$, $2 \mathrm{H}), 7.29(\mathrm{~d}, J=7.6 \mathrm{~Hz}, 1 \mathrm{H}), 7.22(\mathrm{dd}, J=7.6,7.4 \mathrm{~Hz}, 1 \mathrm{H}), 7.16(\mathrm{dd}$, $J=7.5,7.4 \mathrm{~Hz}, 1 \mathrm{H}), 3.70(\mathrm{~s}, 3 \mathrm{H}), 2.50(\mathrm{~s}, 3 \mathrm{H}) ;{ }^{13} \mathbf{C}$ NMR (151 MHz, $\left.\mathbf{C D C l}_{3}\right) \delta 138.87,137.63,136.63,130.97,129.48,125.89,123.66$, $122.09,121.20,120.02,119.83,109.33,29.77,11.48$ (carbon peaks of $-\mathrm{C}_{4} \mathrm{~F}_{8}-$ are omitted due to complicated C-F splitting); ${ }^{19}$ F NMR (564 MHz, $\left.\mathbf{C D C l}_{3}\right) \delta$-86.43, -103.62, -118.95, -121.32; IR (neat): 
$v_{\max }=2927,1559,1476,1173,1118,745 \mathrm{~cm}^{-1} ;$ HRMS m/z (EI) calc. for $\mathrm{C}_{20} \mathrm{H}_{15} \mathrm{~F}_{8} \mathrm{NS}[\mathrm{M}+]$ 453.0797, found 453.0799; $\boldsymbol{R}_{\boldsymbol{f}} 0.50$ (hex/EtOAc, 4/1).

3ae: colorless oil (86.1 mg, 62\%); ${ }^{1} \mathbf{H}$ NMR (600 MHz, $\left.\mathbf{C D C l}_{3}\right) \delta 7.61(\mathrm{~d}, J$

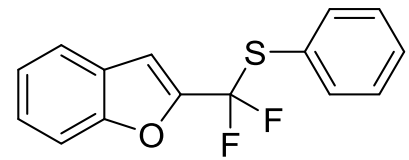
$=7.1 \mathrm{~Hz}, 2 \mathrm{H}), 7.58(\mathrm{t}, J=8.0 \mathrm{~Hz}, 2 \mathrm{H}), 7.43-7.38(\mathrm{~m}, 2 \mathrm{H}), 7.34(\mathrm{t}, J=7.7$ $\mathrm{Hz}, 2 \mathrm{H}), 7.28$ (t, $J=7.7 \mathrm{~Hz}, 1 \mathrm{H}), 6.93(\mathrm{~s}, 1 \mathrm{H}) ;{ }^{13} \mathbf{C}$ NMR (151 MHz, CDCl $\left.\mathbf{3}\right)$ $\delta=155.31,148.62(\mathrm{t}, J=34.7 \mathrm{~Hz}), 136.78,130.45,129.32,126.86,126.43$, $126.34,123.84,123.01\left(\mathrm{t}, J_{\mathrm{C}-\mathrm{F}}=273.8 \mathrm{~Hz}\right), 122.35,112.17,107.52\left(\mathrm{t}, J_{\mathrm{C}-\mathrm{F}}=3.0 \mathrm{~Hz}\right) ;{ }^{19} \mathbf{F} \mathbf{N M R}(\mathbf{3 7 7} \mathbf{~ M H z}$, $\left.\mathbf{C D C l}_{3}\right) \delta-71.40 ;$ IR (neat): $v_{\max }=3063,1475,1290,1253,1163,1053,972,748 \mathrm{~cm}^{-1} ; \boldsymbol{R}_{f} 0.52$ (hex/EtOAc, 8/1).

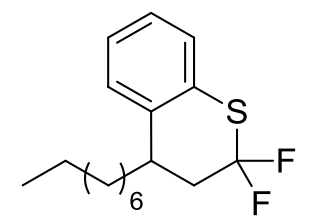

5ac: colorless oil (112.4 mg, 75\%) ; ${ }^{1} \mathbf{H}$ NMR (600 MHz, CDCl3) $\delta 7.23(\mathrm{~d}, J=7.1$ $\mathrm{Hz}, 1 \mathrm{H}), 7.17(\mathrm{~m}, 3 \mathrm{H}), 2.98(\mathrm{~m}, 1 \mathrm{H}), 2.49$ (dddd, $J_{\mathrm{H}-\mathrm{F}}=19.5,14.0, J=12.8,4.0 \mathrm{~Hz}$, $1 \mathrm{H}), 2.39\left(\mathrm{dddd}, J_{\mathrm{H}-\mathrm{F}}=14.0,13.8, J=13.7,7.4 \mathrm{~Hz}, 1 \mathrm{H}\right), 1.83(\mathrm{~m}, 1 \mathrm{H}), 1.72(\mathrm{~m}, 1 \mathrm{H})$, $1.45(\mathrm{~m}, 1 \mathrm{H}), 1.30(\mathrm{~m}, 11 \mathrm{H}), 0.89(\mathrm{t}, J=7.0 \mathrm{~Hz}, 3 \mathrm{H}) ;{ }^{13} \mathbf{C} \mathbf{~ N M R}\left(\mathbf{1 5 1} \mathbf{~ M H z}, \mathbf{C D C l}_{3}\right)$ $\delta=137.93,131.25\left(\mathrm{t}, J_{\mathrm{C}-\mathrm{F}}=268.8 \mathrm{~Hz}\right), 130.31,128.10,127.34,127.22\left(\mathrm{t}, J_{\mathrm{C}-\mathrm{F}}=3.0 \mathrm{~Hz}\right), 126.27,39.72(\mathrm{t}$, $\left.J_{\mathrm{C}-\mathrm{F}}=22.0 \mathrm{~Hz}\right), 38.48,32.08,31.69,29.83,29.71,29.50,27.36,22.89,14.33 ;{ }^{19} \mathbf{F}$ NMR (564 MHz, $\left.\mathbf{C D C l}_{3}\right) \delta-61.63(\mathrm{~d}, J=217.0 \mathrm{~Hz}),-62.37(\mathrm{~d}, J=216.9 \mathrm{~Hz})$; IR (neat): $v_{\max }=2924,2854,1467,1163$, 1016, $750 \mathrm{~cm}^{-1}$; HRMS m/z (EI) calc. for $\mathrm{C}_{17} \mathrm{H}_{24} \mathrm{~F}_{2} \mathrm{~S}[\mathrm{M}+]$ 298.1567, found 298.1569; $\boldsymbol{R}_{\boldsymbol{f}} 0.74$ (hex/EtOAc, 1/8).

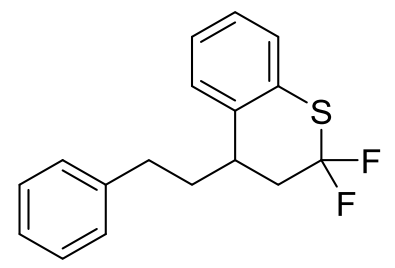

5ad: colorless oil (110.8 mg, 76\%); ${ }^{1} \mathrm{H}$ NMR (600 MHz, CDCl3) $\delta 7.28(\mathrm{~m}$, 2H), $7.19(\mathrm{~m}, 4 \mathrm{H}), 7.15(\mathrm{~m}, 3 \mathrm{H}), 3.00(\mathrm{ddt}, J=10.0,7.8,3.9 \mathrm{~Hz}, 1 \mathrm{H}), 2.75$ $\left(\mathrm{ddd}, J_{\mathrm{H}-\mathrm{F}}=14.3,10.0, J=5.7 \mathrm{~Hz}, 1 \mathrm{H}\right), 2.63\left(\mathrm{ddd}, J_{\mathrm{H}-\mathrm{F}}=13.9,10.0, J=6.3 \mathrm{~Hz}\right.$, 1H), $2.48(\mathrm{~m}, 2 \mathrm{H}), 2.13(\mathrm{~m}, 1 \mathrm{H}), 2.05(\mathrm{~m}, 1 \mathrm{H}) ;{ }^{13} \mathbf{C}$ NMR (151 MHz, CDCl $\left.\mathbf{3}\right)$ $\delta 141.57,137.33,131.12\left(\mathrm{t}, J_{\mathrm{C}-\mathrm{F}}=268.6 \mathrm{~Hz}\right), 130.19,128.71,128.55,128.32$, 127.52, $127.26\left(\mathrm{t}, J_{\mathrm{C}-\mathrm{F}}=3 \mathrm{~Hz}\right), 126.32,126.26,39.40\left(\mathrm{t}, J_{\mathrm{C}-\mathrm{F}}=22.1 \mathrm{~Hz}\right), 38.03,33.45,33.39 ;{ }^{19} \mathbf{F} \mathbf{~ N M R}$ $\left(377 \mathbf{~ M H z}, \mathbf{C D C l}_{3}\right) \delta-60.50\left(\mathrm{~d}, J_{\mathrm{F}-\mathrm{F}}=218.7 \mathrm{~Hz}\right),-62.00(\mathrm{~d}, \mathrm{JF}-\mathrm{F}=218.7 \mathrm{~Hz}) ; \mathbf{I R}\left(\right.$ neat): $v_{\max }=3027$, 2931, 1603, 1496, 1161, 1016, 905, 728, $698 \mathrm{~cm}^{-1}$; HRMS m/z (EI) calc. for $\mathrm{C}_{17} \mathrm{H}_{16} \mathrm{~F}_{2} \mathrm{~S}$ [M+] 290.0941, found $290.0939 ; \boldsymbol{R}_{f} 0.58$ (hex/EtOAc, 1/8).

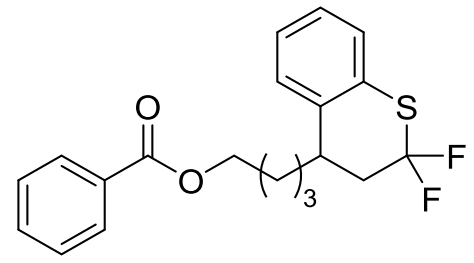

5ae: colorless oil (86.9 mg, 80\%); ${ }^{1}$ H NMR (600 MHz, CDCl3) $\delta 8.03$ $(\mathrm{dd}, J=8.4,1.3 \mathrm{~Hz}, 2 \mathrm{H}), 7.56(\mathrm{tt}, J=7.6,1.3 \mathrm{~Hz}, 1 \mathrm{H}), 7.44(\mathrm{dd}, J=8.4$, 
$7.6 \mathrm{~Hz}, 2 \mathrm{H}), 7.22(\mathrm{~d}, J=7.5 \mathrm{~Hz}, 1 \mathrm{H}), 7.17(\mathrm{~m}, 3 \mathrm{H}), 4.35(\mathrm{t}, J=6.5 \mathrm{~Hz}, 2 \mathrm{H}), 3.01(\mathrm{~m}, 1 \mathrm{H}), 2.48(\mathrm{~m}, 2 \mathrm{H})$, $1.92\left(\mathrm{dddd}, J_{\mathrm{H}-\mathrm{F}}=18.4,9.8, J=6.3,2.1 \mathrm{~Hz}, 1 \mathrm{H}\right), 1.84(\mathrm{~m}, 3 \mathrm{H}), 1.62(\mathrm{~m}, 1 \mathrm{H}), 1.50(\mathrm{~m}, 1 \mathrm{H}) ;{ }^{13} \mathbf{C} \mathbf{~ N M R}$ $\left(151 \mathrm{MHz}, \mathbf{C D C l}_{3}\right) \delta=166.81,137.36,133.09,131.11\left(\mathrm{t}, J_{\mathrm{C}-\mathrm{F}}=268.6 \mathrm{~Hz}\right), 130.57,130.18,128.27$, 127.51, $127.28\left(\mathrm{t}, J_{\mathrm{C}-\mathrm{F}}=3.1 \mathrm{~Hz}\right), 126.30,64.87,39.65\left(\mathrm{t}, J_{\mathrm{C}-\mathrm{F}}=22.1 \mathrm{~Hz}\right), 38.56,31.27,28.89,24.00 ;{ }^{19} \mathbf{F}$ NMR (564 MHz, CDCl 3 ) $\delta-61.05(\mathrm{~d}, J=217.6 \mathrm{~Hz}),-62.21(\mathrm{~d}, J=217.6 \mathrm{~Hz})$; IR (neat): $v_{\max }=2944$, 2867, 1716, 1451, 1273, 1113, 1025, $711 \mathrm{~cm}^{-1}$; HRMS m/z (EI) calc. for $\mathrm{C}_{20} \mathrm{H}_{20} \mathrm{~F}_{2} \mathrm{O}_{2} \mathrm{~S}[\mathrm{M}+] 362.1152$, found $362.1155 ; \boldsymbol{R}_{f} 0.54$ (hex/EtOAc, 1/4).

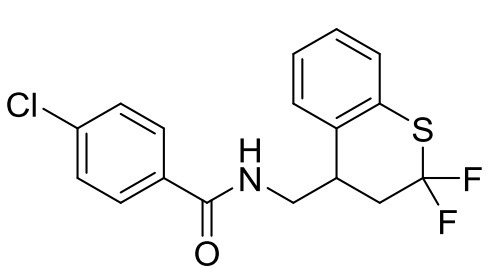

5af: colorless oil (73.72 mg, 70\%); ${ }^{1}$ H NMR (600 MHz, CDCl3) $\delta$ $7.68(\mathrm{~d}, J=8.6 \mathrm{~Hz}, 2 \mathrm{H}), 7.41(\mathrm{~d}, J=8.6 \mathrm{~Hz}, 2 \mathrm{H}), 7.28(\mathrm{~d}, J=7.3 \mathrm{~Hz}$, $1 \mathrm{H}), 7.24(\mathrm{dd}, J=7.5,7.3 \mathrm{~Hz}, 1 \mathrm{H}), 7.17(\mathrm{~m}, 2 \mathrm{H}), 6.39(\mathrm{~s}, 1 \mathrm{H}), 3.82(\mathrm{~m}$, $1 \mathrm{H}), 3.65(\mathrm{~m}, 1 \mathrm{H}), 3.56$ (dddd, $J=8.5,8.0,7.9,4.6 \mathrm{~Hz}, 1 \mathrm{H}), 2.69$ $\left(\mathrm{dddd}, J_{H-F}=14.5,14.4, J=8.5,4.6 \mathrm{~Hz}, 1 \mathrm{H}\right), 2.59\left(\mathrm{dddd}, J_{H-F}=27.5\right.$, 14.4, $J=8.5,4.6 \mathrm{~Hz}, 1 \mathrm{H}) ;{ }^{13} \mathbf{C}$ NMR (151 MHz, $\left.\mathbf{C D C l}_{3}\right) \delta=166.96,138.25,133.62,132.66,130.52(\mathrm{t}$, $\left.J_{\mathrm{C}-\mathrm{F}}=267.8 \mathrm{~Hz}\right), 130.17,129.53,129.16,128.50,128.30,127.04\left(\mathrm{dd}, J_{\mathrm{C}-\mathrm{F}}=4.5,3.0 \mathrm{~Hz}\right), 126.51,41.75$, $38.10\left(\mathrm{~d}, J_{\mathrm{C}-\mathrm{F}}=6.7 \mathrm{~Hz}\right), 37.06\left(\mathrm{t}, J_{\mathrm{C}-\mathrm{F}}=22.4 \mathrm{~Hz}\right) ;{ }^{19} \mathbf{F} \mathbf{N M R}\left(\mathbf{5 6 4} \mathbf{~ M H z}, \mathbf{C D C l}_{3}\right) \delta-58.81(\mathrm{~d}, J=221.6$ $\mathrm{Hz}),-64.13\left(\mathrm{~d}, J=221.5 \mathrm{~Hz}\right.$ ); IR (neat): $v_{\max }=3306,3071,2925,1635,1538,1486,1312,1093,1015$, 846, $755 \mathrm{~cm}^{-1}$; HRMS m/z (EI) calc. for $\mathrm{C}_{17} \mathrm{H}_{14} \mathrm{ClF}_{2} \mathrm{NOS}[\mathrm{M}+]$ 353.0453, found 353.0451; $\boldsymbol{R}_{\boldsymbol{f}} 0.45$ (hex/EtOAc, 1/2).
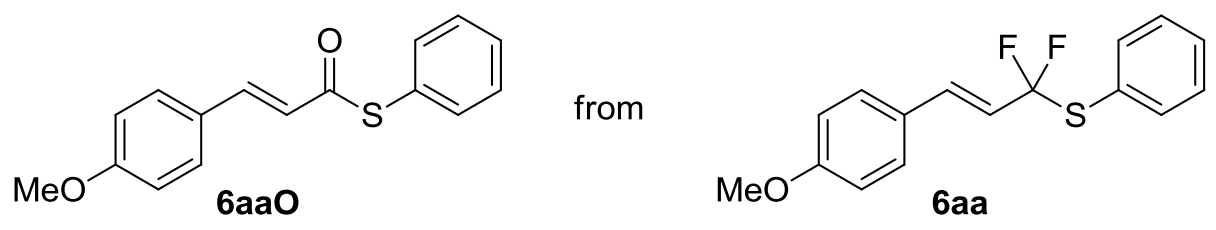

6aa: ${ }^{19}$ F NMR (377 MHz, $\left.\mathrm{CDCl}_{3}\right) \delta-70.95$

6aaO: White solid (109.9 mg, 81\%); ${ }^{1} \mathbf{H}$ NMR (600 MHz, $\left.\mathbf{C D C l}_{3}\right) \delta 7.66(\mathrm{~d}, J=15.7 \mathrm{~Hz}, 1 \mathrm{H}), 7.51(\mathrm{~m}$, 4H), $7.45(\mathrm{~m}, 3 \mathrm{H}), 6.93(\mathrm{~d}, J=8.8 \mathrm{~Hz}, 2 \mathrm{H}), 6.68(\mathrm{~d}, J=15.7 \mathrm{~Hz}, 1 \mathrm{H}), 3.85(\mathrm{~s}, 3 \mathrm{H}) ;{ }^{13} \mathbf{C}$ NMR (151 MHz, $\left.\mathbf{C D C l}_{3}\right) \delta=187.90,161.99,141.47,134.79,130.44,129.46,129.29,128.09,126.82,121.93,114.63$, 55.56; IR (neat): $v_{\max }=3058,3000,2838,1664,1593,1509,1249,1173,1020,804,745,722,689 \mathrm{~cm}^{-1}$; $\boldsymbol{R}_{f} 0.46$ (hex/EtOAc, 4/1). 


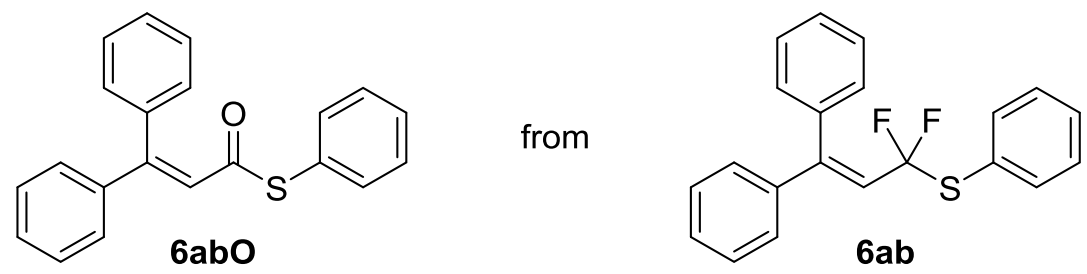

6ab: ${ }^{19} \mathrm{~F}$ NMR (377 $\left.\mathrm{MHz}, \mathrm{CDCl}_{3}\right) \delta-62.53$

6abO: White solid (142.2 mg, 90\%); ${ }^{1} \mathbf{H}$ NMR (600 MHz, $\left.\mathbf{C D C l}_{3}\right) \delta 7.34$ (m, 13H), 7.24 (m, 2H), 6.66(s, 1H); ${ }^{13} \mathbf{C}$ NMR (151 MHz, $\left.\mathbf{c d c l}_{3}\right) \delta=187.00,154.68,140.86,138.58,134.67,130.01,129.71,129.36$, 129.25, 128.89, 128.80, 128.66, 128.39, 128.17, 123.15; IR (neat): $v_{\max }=3056,2926,1687,1570,1440$, $1017,954,691 \mathrm{~cm}^{-1} ; \boldsymbol{R}_{f} 0.61$ (hex/EtOAc, 1/4).

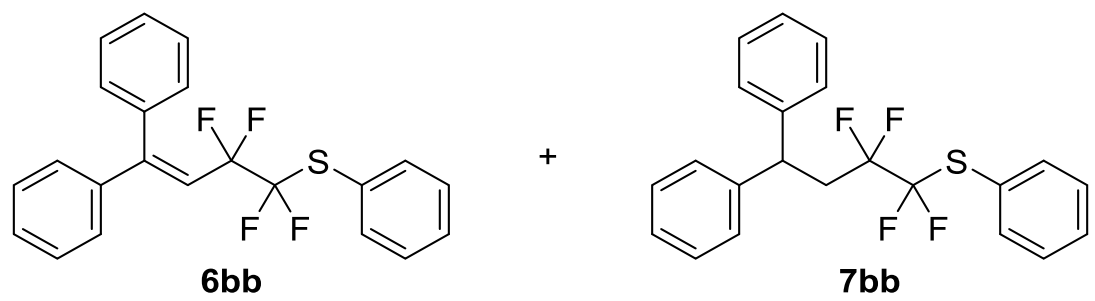

6bb $(102.9 \mathrm{mg}, 53 \%)+\mathbf{7 b b}(33.1 \mathrm{mg}, 17 \%)$ : colorless oil (136 mg, 70\%); The yields was determined by GC-Mass and ${ }^{1} \mathrm{H}$ NMR) ; ${ }^{1} \mathbf{H}$ NMR (600 MHz, $\left.\mathbf{C D C l}_{3}\right)$ 6bb: $\delta 7.63-7.57$ (m, 2H), $7.44-7.38$ (m, 1H), $7.36-7.32(\mathrm{~m}, 4 \mathrm{H}), 7.33-7.21(\mathrm{~m}, 8 \mathrm{H}), 6.16\left(\mathrm{t}, J_{\mathrm{H}-\mathrm{F}}=14.4 \mathrm{~Hz}, 1 \mathrm{H}\right) ; 7 \mathbf{b b}: \delta 7.63-7.57(\mathrm{~m}, 2 \mathrm{H}), 7.44-$ $7.38(\mathrm{~m}, 1 \mathrm{H}), 7.36-7.32(\mathrm{~m}, 4 \mathrm{H}), 7.33-7.21(\mathrm{~m}, 6 \mathrm{H}), 7.19-7.15(\mathrm{~m}, 2 \mathrm{H}), 4.45\left(\mathrm{td}, J_{\mathrm{H}-\mathrm{F}}=3.0, J=7.1\right.$ $\mathrm{Hz}, 1 \mathrm{H}), 2.90\left(\mathrm{td}, J_{\mathrm{H}-\mathrm{F}}=18.4, J=7.0 \mathrm{~Hz}, 2 \mathrm{H}\right) ;{ }^{13} \mathbf{C} \mathbf{N M R}\left(\mathbf{1 5 1} \mathbf{~ M H z}, \mathbf{C D C l}_{\mathbf{3}}\right) \delta 153.14,143.77,141.31$, 138.10, 137.29, 137.23, 130.63, 130.55, 129.41, 129.36, 128.86, 128.60, 128.29, 128.14, 127.96, 127.76, $126.82,124.91,124.66,124.44,124.15,122.75,118.86,115.65,114.66,44.32(7 \mathbf{b b}), 36.73\left(\mathrm{t}, J_{\mathrm{C}-\mathrm{F}}=21.4\right.$ $\mathrm{Hz}$ ) (7bb) (carbon peaks of $-\mathrm{C}_{2} \mathrm{~F}_{4}-$ are omitted due to complicated C-F splitting); ${ }^{19} \mathbf{F}$ NMR (377 MHz, $\mathbf{C D C l}_{3}$ ) $\delta$-89.92, -89.98, -103.15 (6bb), -111.81 (7bb); IR (neat): $v_{\max }=3060,3029,1635,1442,1237$, 1066, 928, 748, $690 \mathrm{~cm}^{-1}$; HRMS m/z (EI) calc. for $\mathrm{C}_{22} \mathrm{H}_{16} \mathrm{~F}_{4} \mathrm{~S}$ [M+] 388.0909, found 388.0906; $\boldsymbol{R}_{f} 0.61$ (hex/EtOAc, 8/1).<smiles>FC(F)(F)C(F)(F)C(F)(F)C(F)(F)C(F)(F)C(F)(F)Sc1ccccc1</smiles> 
6cb (124.5 mg, $51 \%)+7$ cb (56.4 mg, 23\%): colorless oil(180.9 mg, 74\%); The yields was determined by GC-Mass and ${ }^{1} \mathrm{H}$ NMR); ${ }^{1} \mathbf{H}$ NMR (600 MHz, $\left.\mathbf{C D C l}_{3}\right)$ 6cb: $\delta 7.69-7.63(\mathrm{~m}, 2 \mathrm{H}), 7.53-7.46(\mathrm{~m}$, $1 \mathrm{H}), 7.45-7.23(\mathrm{~m}, 12 \mathrm{H}), 6.12\left(\mathrm{t}, J_{\mathrm{H}-\mathrm{F}}=14.6 \mathrm{~Hz}, 1 \mathrm{H}\right) ; \mathbf{c b}: \delta 7.69-7.63(\mathrm{~m}, 2 \mathrm{H}), 7.53-7.46(\mathrm{~m}, 1 \mathrm{H})$, $7.45-7.23(\mathrm{~m}, 10 \mathrm{H}), 7.23-7.19(\mathrm{~m}, 2 \mathrm{H}) 4.48(\mathrm{t}, J=7.1 \mathrm{~Hz}, 1 \mathrm{H}), 2.90\left(\mathrm{td}, J_{\mathrm{H}-\mathrm{F}}=18.5, J=7.1 \mathrm{~Hz}, 2 \mathrm{H}\right)$; ${ }^{13}$ C NMR (151 MHz, $\mathbf{C D C l}_{3}$ ) $\delta$ 153.84, 143.48, 141.11, 137.86, 137.61, 131.05, 129.51, 129.27, 129.26, $128.92,128.66,128.39,128.13,128.00,127.71,126.94,123.43,113.68,43.97(7 \mathbf{c b}), 36.56\left(\mathrm{t}, J_{\mathrm{C}-\mathrm{F}}=21.1\right.$ $\mathrm{Hz})(7 \mathbf{c b})$ (carbon peaks of $-\mathrm{C}_{4} \mathrm{~F}_{8}-$ are omitted due to complicated C-F splitting); ${ }^{19} \mathbf{F}$ NMR (564 MHz, $\mathbf{C D C l}_{3}$ ) 6cb: $\delta-86.58,-103.62,-118.57,-122.54 ;$ 7cb: $-86.5849,-112.82,-118.79,-123.02$; IR (neat): $v_{\max }=3061,1637,1444,1171,1117,750,690 \mathrm{~cm}^{-1}$; HRMS m/z (EI) calc. for $\mathrm{C}_{24} \mathrm{H}_{16} \mathrm{~F}_{8} \mathrm{~S}[\mathrm{M}+]$ 488.0845, found $488.0848 ; \boldsymbol{R}_{f} 0.59$ (hex/EtOAc, 8/1).

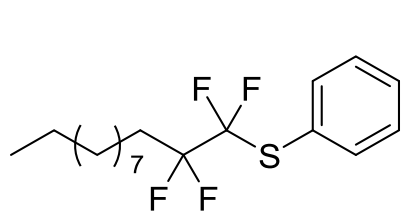

7bc: colorless oil (98.1 mg, 56\%) ; ${ }^{1} \mathbf{H}$ NMR (600 MHz, $\left.\mathbf{C D C l}_{3}\right) \delta 7.66(\mathrm{~d}, J$ $=8.0 \mathrm{~Hz}, 2 \mathrm{H}), 7.47(\mathrm{t}, J=7.5 \mathrm{~Hz}, 1 \mathrm{H}), 7.41(\mathrm{dd}, J=8.0,7.5 \mathrm{~Hz}, 2 \mathrm{H}), 2.02-$ $2.11\left(\mathrm{tt}, J_{\mathrm{H}-\mathrm{F}}=18.2, J=8.0 \mathrm{~Hz}, 2 \mathrm{H}\right), 1.59(\mathrm{tt}, J=8.2,7.2 \mathrm{~Hz}, 2 \mathrm{H}), 1.42-$ $\left.1.21(\mathrm{~m}, 14 \mathrm{H}), 0.89(\mathrm{t}, J=7.1 \mathrm{~Hz}, 3 \mathrm{H}) ;{ }^{13} \mathbf{C ~ N M R ~ ( 1 5 1 ~ M H z , ~} \mathbf{C D C l}_{3}\right) \delta$ 138.41, 137.30, 130.55, 129.35, 32.14, 31.36 (t, $\left.J_{\mathrm{C}-\mathrm{F}}=22.9 \mathrm{~Hz}\right), 29.96,29.78,29.67,29.54,29.49,22.92$, 20.85, 14.33 (carbon peaks of $-\mathrm{C}_{2} \mathrm{~F}_{4}-$ are omitted due to complicated C-F splitting); ${ }^{19} \mathbf{F}$ NMR (377 $\mathbf{~ M H z}$, $\left.\mathbf{C D C l}_{3}\right) \delta$-88.81, -112.73; IR (neat): $v_{\max }=2924,2854,1467,1240,1092,1068,924,749,690 \mathrm{~cm}^{-1}$; HRMS m/z (EI) calc. for $\mathrm{C}_{18} \mathrm{H}_{26} \mathrm{~F}_{4} \mathrm{~S}$ [M+] 350.1691, found 350.1692; $\boldsymbol{R}_{f} 0.58$ (hex).

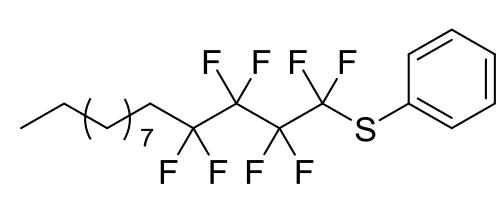

7cc: colorless oil (121.6 mg, 54\%); ${ }^{\mathbf{1}} \mathbf{H}$ NMR (600 $\left.\mathbf{M H z}, \mathbf{C D C l}_{3}\right) \delta 7.67$ $(\mathrm{d}, J=7.3 \mathrm{~Hz}, 2 \mathrm{H}), 7.49(\mathrm{t}, J=7.5 \mathrm{~Hz}, 1 \mathrm{H}), 7.42(\mathrm{dd}, J=7.5,7.3 \mathrm{~Hz}$, $2 \mathrm{H}), 2.03\left(\mathrm{tt}, J_{\mathrm{H}-\mathrm{F}}=18, J=8.2 \mathrm{~Hz}, 2 \mathrm{H}\right), 1.59(\mathrm{tt}, J=8.2,8.1 \mathrm{~Hz}, 2 \mathrm{H})$, $1.42-1.21(\mathrm{~m}, 14 \mathrm{H}), 0.89(\mathrm{t}, J=7.0 \mathrm{~Hz}, 3 \mathrm{H}) ;{ }^{13} \mathbf{C}$ NMR (151 MHz, $\left.\mathbf{C D C l}_{3}\right) \delta 139.05,137.62,131.05,129.51,32.11,31.26\left(\mathrm{t}, J_{\mathrm{C}-\mathrm{F}}=22.3 \mathrm{~Hz}\right), 29.75,29.62,29.51,29.47$, 29.38, 22.90, 20.40, 14.32 (carbon peaks of $-\mathrm{C}_{4} \mathrm{~F}_{8}-$ are omitted due to complicated C-F splitting); ${ }^{19} \mathbf{F}$ NMR (564 MHz, $\mathbf{C D C l}_{3}$ ) $\delta$-86.68, -114.45, -119.08, -123.08; IR (neat): $v_{\max }=2926,2857,1469,1443$, 1172, 1118, 750, $689 \mathrm{~cm}^{-1}$; HRMS m/z (EI) calc. for $\mathrm{C}_{20} \mathrm{H}_{26} \mathrm{~F}_{8} \mathrm{~S}[\mathrm{M}+]$ 450.1627, found 450.1630; $\boldsymbol{R}_{\boldsymbol{f}}$ 0.61 (hex).

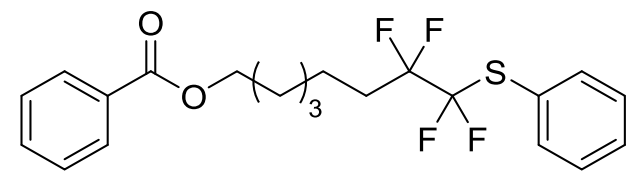

7be: colorless oil (130.5 mg, 63\%); ${ }^{1} \mathbf{H}$ NMR (600 MHz, $\left.\mathbf{C D C l}_{3}\right) \delta 8.05(\mathrm{~d}, J=7.6 \mathrm{~Hz}, 2 \mathrm{H}), 7.65(\mathrm{~d}, J=8.2 \mathrm{~Hz}, 2 \mathrm{H})$, $7.56(\mathrm{t}, J=7.5 \mathrm{~Hz}, 1 \mathrm{H}), 7.49-7.41(\mathrm{~m}, 3 \mathrm{H}), 7.40(\mathrm{dd}, J=7.6$, 
$7.5 \mathrm{~Hz}, 2 \mathrm{H}), 4.33(\mathrm{t}, J=6.6 \mathrm{~Hz}, 2 \mathrm{H}), 2.07\left(\mathrm{tt}, J_{\mathrm{H}-\mathrm{F}}=18.0, J=8.3 \mathrm{~Hz}, 2 \mathrm{H}\right), 1.82-1.76(\mathrm{~m}, 2 \mathrm{H}), 1.66-$ 1.60 (m, 2H), 1.52 - 1.43 (m, 4H); $\left.{ }^{13} \mathbf{C ~ N M R ~ ( 1 5 1 ~ M H z , ~ C D C l ~}\right) \delta 166.84,137.28,133.05,130.64$, 130.58, 129.74, 129.36, 128.55, 124.36, 65.05, $31.24\left(\mathrm{t}, J_{\mathrm{C}-\mathrm{F}}=22.9 \mathrm{~Hz}\right), 29.09,28.73,26.00,20.79$ (carbon peaks of $-\mathrm{C}_{2} \mathrm{~F}_{4}-$ are omitted due to complicated C-F splitting); ${ }^{19} \mathbf{F}$ NMR (564 MHz, $\left.\mathbf{C D C l}\right) \delta$ 88.29, -112.13; IR (neat): $v_{\max }=2942,2860,1716,1271,1096,1068,921,711 \mathrm{~cm}^{-1} ; \mathbf{H R M S ~ m / z ~ ( E I ) ~}$ calc. for $\mathrm{C}_{21} \mathrm{H}_{22} \mathrm{~F}_{4} \mathrm{O}_{2} \mathrm{~S}[\mathrm{M}+]$ 414.1277, found 414.1279; $\boldsymbol{R}_{\boldsymbol{f}} 0.48$ (hex/EtOAc, 8/1).

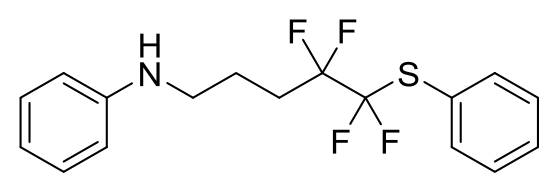

7bg: brown oil (89.2 mg, 52\%); ${ }^{1} \mathbf{H}$ NMR (600 MHz, $\left.\mathbf{C D C l}_{3}\right) \delta 7.65$ $(\mathrm{d}, J=8.0 \mathrm{~Hz}, 2 \mathrm{H}), 7.46(\mathrm{tt}, J=7.6,1.6 \mathrm{~Hz}, 1 \mathrm{H}), 7.41(\mathrm{dd}, J=7.6$, $8.0 \mathrm{~Hz}, 2 \mathrm{H}), 7.19$ (dd, $J=8.6,7.1 \mathrm{~Hz}, 2 \mathrm{H}), 6.73$ (tt, $J=7.1,1.0 \mathrm{~Hz}$, $1 \mathrm{H}), 6.62(\mathrm{dd}, J=8.6,1.0 \mathrm{~Hz}, 2 \mathrm{H}), 3.23(\mathrm{t}, J=7.0 \mathrm{~Hz}, 2 \mathrm{H}), 2.21(\mathrm{tt}$, $\left.J_{\mathrm{H}-\mathrm{F}}=18, J=8.0 \mathrm{~Hz}, 2 \mathrm{H}\right), 1.93(\mathrm{tt}, J=8.0,7.0 \mathrm{~Hz}, 2 \mathrm{H}) ;{ }^{13} \mathbf{C}$ NMR $\left(\mathbf{1 5 1} \mathbf{~ M H z}, \mathbf{C D C l}_{3}\right) \delta 148.15,137.31$, 130.66, 129.54, 129.40, 124.21, 117.84, 113.01, 43.47, 29.05 (t, $J_{\mathrm{C}-\mathrm{F}}=23.1 \mathrm{~Hz}$ ), 21.31 (carbon peaks of $\mathrm{C}_{2} \mathrm{~F}_{4}-$ are omitted due to complicated C-F splitting); ${ }^{19} \mathbf{F}$ NMR (377 $\left.\mathbf{M H z}, \mathbf{C D C l}_{3}\right) \delta$-88.55, -112.25; IR (neat): $v_{\max }=3401,3056,2933,1604,1507,1259,1093,924,749,691 \mathrm{~cm}^{-1} ;$ HRMS m/z (EI) calc. for $\mathrm{C}_{17} \mathrm{H}_{17} \mathrm{~F}_{4} \mathrm{NS}[\mathrm{M}+]$ 343.1018, found 343.1021; $\boldsymbol{R}_{\boldsymbol{f}} 0.55$ (hex/EtOAc, 8/1).

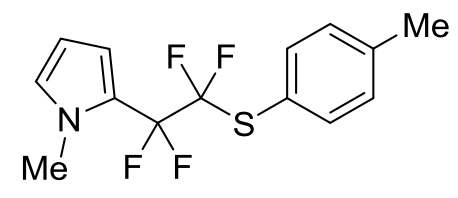

9: colorless oil (98.5 mg, 65\%); ${ }^{1} \mathrm{H}$ NMR (600 MHz, CDCl3) $\delta 7.52(\mathrm{~d}, J$ $=8.1 \mathrm{~Hz}, 2 \mathrm{H}), 7.20(\mathrm{~d}, J=8.1 \mathrm{~Hz}, 2 \mathrm{H}), 6.72-6.70(\mathrm{~m}, 1 \mathrm{H}), 6.59(\mathrm{~d}, J=$ $2.4 \mathrm{~Hz}, 1 \mathrm{H}), 6.13(\mathrm{dd}, J=3.9,2.4 \mathrm{~Hz}, 1 \mathrm{H}), 3.72(\mathrm{~s}, 3 \mathrm{H}), 2.38(\mathrm{~s}, 3 \mathrm{H}) \cdot{ }^{13} \mathrm{C}$ NMR (151 MHz, $\left.\mathbf{C D C l}_{3}\right) \delta 141.01,137.23,130.17,127.55,121.02$, $120.48\left(\mathrm{t}, J_{\mathrm{C}-\mathrm{F}}=29.7 \mathrm{~Hz}\right), 114.14,107.69,36.05,21.53$ (carbon peaks of $-\mathrm{C}_{2} \mathrm{~F}_{4}-$ are omitted due to complicated C-F splitting); ${ }^{19}$ F NMR (564 MHz, CDCl 3 ) $\delta-86.50,-101.62$; IR (neat): $v_{\max }=2925,2859$, 1660, 1492, 1318, 1096, 1078, 811, $731 \mathrm{~cm}^{-1}$; HRMS m/z (EI) calc. for $\mathrm{C}_{14} \mathrm{H}_{13} \mathrm{~F}_{4} \mathrm{NS}$ [M+] 303.0705, found $303.0707 ; \boldsymbol{R}_{f} 0.55$ (hex/EtOAc, 8/1).

11: colorless oil (100.3 mg, 62\%); ${ }^{1} \mathbf{H}$ NMR (600 MHz, CDCl3) $\delta 7.56(\mathrm{~d}$,

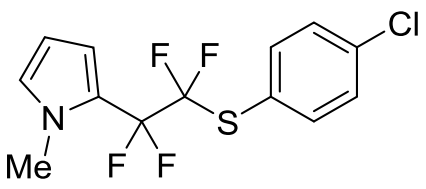
$J=8.5 \mathrm{~Hz}, 2 \mathrm{H}), 7.37(\mathrm{~d}, J=8.5 \mathrm{~Hz}, 2 \mathrm{H}), 6.73-6.70(\mathrm{~m}, 1 \mathrm{H}), 6.58(\mathrm{~d}, J=$ $3.2 \mathrm{~Hz}, 1 \mathrm{H}), 6.14(\mathrm{dd}, J=3.2,3.1 \mathrm{~Hz}, 1 \mathrm{H}), 3.71(\mathrm{~s}, 3 \mathrm{H}) ;{ }^{13} \mathbf{C}$ NMR (151 MHz, $\left.\mathbf{C D C l}_{3}\right) \delta 138.40,137.34,129.64,127.71,123.10,120.17\left(\mathrm{t}, J_{C-F}=\right.$ $29.6 \mathrm{~Hz}$ ), 114.23, 107.78, 36.05 (carbon peaks of $-\mathrm{C}_{2} \mathrm{~F}_{4-}$ are omitted due to complicated C-F splitting); ${ }^{19}$ F NMR (564 MHz, CDCl 3 ) $\delta$-86.20, -101.60; IR (neat): $v_{\max }=2363,2342,1477,1096,1080,907$, 824, $732 \mathrm{~cm}^{-1} ; \mathbf{H R M S ~ m / z ~ ( E I ) ~ c a l c . ~ f o r ~} \mathrm{C}_{13} \mathrm{H}_{10} \mathrm{ClF}_{4} \mathrm{NS}[\mathrm{M}+]$ 323.0159, found 323.0157; $\boldsymbol{R}_{\boldsymbol{f}} 0.60$ (hex/EtOAc, 8/1). 


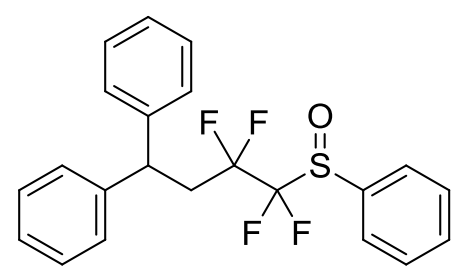

S1: colorless oil (132.0 mg, 65\%); ${ }^{1} \mathbf{H}$ NMR (600 MHz, CDCl $) \delta 7.61(\mathrm{~d}$, $J=7.5 \mathrm{~Hz}, 2 \mathrm{H}), 7.47-7.42(\mathrm{~m}, 5 \mathrm{H}), 7.38(\mathrm{dd}, J=7.5,7.4 \mathrm{~Hz}, 2 \mathrm{H}), 7.32$ $(\mathrm{dd}, J=7.7,7.4 \mathrm{~Hz}, 4 \mathrm{H}), 7.24(\mathrm{t}, J=7.4 \mathrm{~Hz}, 2 \mathrm{H}), 3.18\left(\mathrm{t}, J_{\mathrm{H}-\mathrm{F}}=18.2 \mathrm{~Hz}\right.$, 2H), 2.78 - 2.81 (m, 1H); ${ }^{13} \mathbf{C}$ NMR (151 MHz, $\left.\mathbf{C D C l}_{3}\right) \delta$ 146.01, 137.32, $130.71,129.43,128.52,127.53,125.71,124.02,76.85,41.42\left(\mathrm{t}, J_{\mathrm{C}-\mathrm{F}}=\right.$

$19.3 \mathrm{~Hz}$ ); ${ }^{19} \mathbf{F}$ NMR (564 MHz, $\left.\mathbf{C D C l}_{3}\right) \delta-89.17,-106.62$; IR (neat): $v_{\max }=3061,1598,1449,1367$, 1237, 1201, 1067, 908, 749, $700 \mathrm{~cm}^{-1}$; HRMS m/z (EI) calc. for $\mathrm{C}_{22} \mathrm{H}_{18} \mathrm{~F}_{4} \mathrm{OS}$ [M+] 406.1014, found $406.1017 ; \boldsymbol{R}_{f} 0.48$ (hex/EtOAc, 4/1).

\section{References}

S1. Gaussian 09, Revision E.01, Frisch, M. J.; Trucks, G. W.; Schlegel, H. B.; Scuseria, G. E.; Robb, M. A.; Cheeseman, J. R.; Scalmani, G.; Barone, V.; Mennucci, B.; Petersson, G. A.; Nakatsuji, H.; Caricato, M.; Li, X.; Hratchian, H. P.; Izmaylov, A. F.; Bloino, J.; Zheng, G.; Sonnenberg, J. L.; Hada, M.; Ehara, M.; Toyota, K.; Fukuda, R.; Hasegawa, J.; Ishida, M.; Nakajima, T.; Honda, Y.; Kitao, O.; Nakai, H.; Vreven, T.; Jr., Montgomery, J. A.; Peralta, J. E.; Ogliaro, F.; Bearpark, M.; Heyd, J. J.; Brothers, E.; Kudin, K. N.; Staroverov, V. N.; Kobayashi, R.; Normand, J.; Raghavachari, K.; Rendell, A.; Burant, J. C.; Iyengar, S. S.; Tomasi, J.; Cossi, M.; Rega, N.; J. Millam, M.; Klene, M.; Knox, J. E.; Cross, J. B.; Bakken, V.; Adamo, C.; Jaramillo, J.; Gomperts, R.; Stratmann, R. E.; Yazyev, O.; Austin, A. J.; Cammi, R.; Pomelli, C.; Ochterski, J. W.; Martin, R. L.; Morokuma, K.; Zakrzewski, V. G.; Voth, G. A.; Salvador, P.; Dannenberg, J. J.; Dapprich, S.; Daniels, A. D.; Farkas, Ö.; Foresman, J. B.; Ortiz, J. V.; Cioslowski, J.; Fox, D. J. Gaussian, Inc., Wallingford CT, 2009.

S2. Denis, P. A.; J. Chem. Theory Comput. 2005, 1, 900

S3. Bergès, J.; Varmenot, N.; Scemama, A.; Abedinzadeh, Z.; Bobrowski, K. J. Phys. Chem. A, 2008, 112,7015

S4. Tomasi, J.; Mennucci, B.; Cammi, R. Chem. Rev. 2005, 105, 2999

S5. NBO Version 3.1, E. D. Glendening, Reed, A. E.; Carpenter, J. E.; Weinhold. F.

S6. Kuhakarn, C.; Surapanich, N.; Kamtonwong, S.; Pohmakotr, M.; Reutrakul, V. Eur. J. Org. Chem. 2011, 2011, 5911

S7. A mechanistic study proved that the catalytic cycle with $f a c-\operatorname{Ir}^{\mathrm{III}}(\mathrm{ppy})_{3}$ utilizes the oxidative quenching pathway in difluoroalkylation reactions, see: Jung, J.; Kim, E.; You, Y.; Cho, E. J. Adv. Synth. Catal. 2014, 356, 2741 


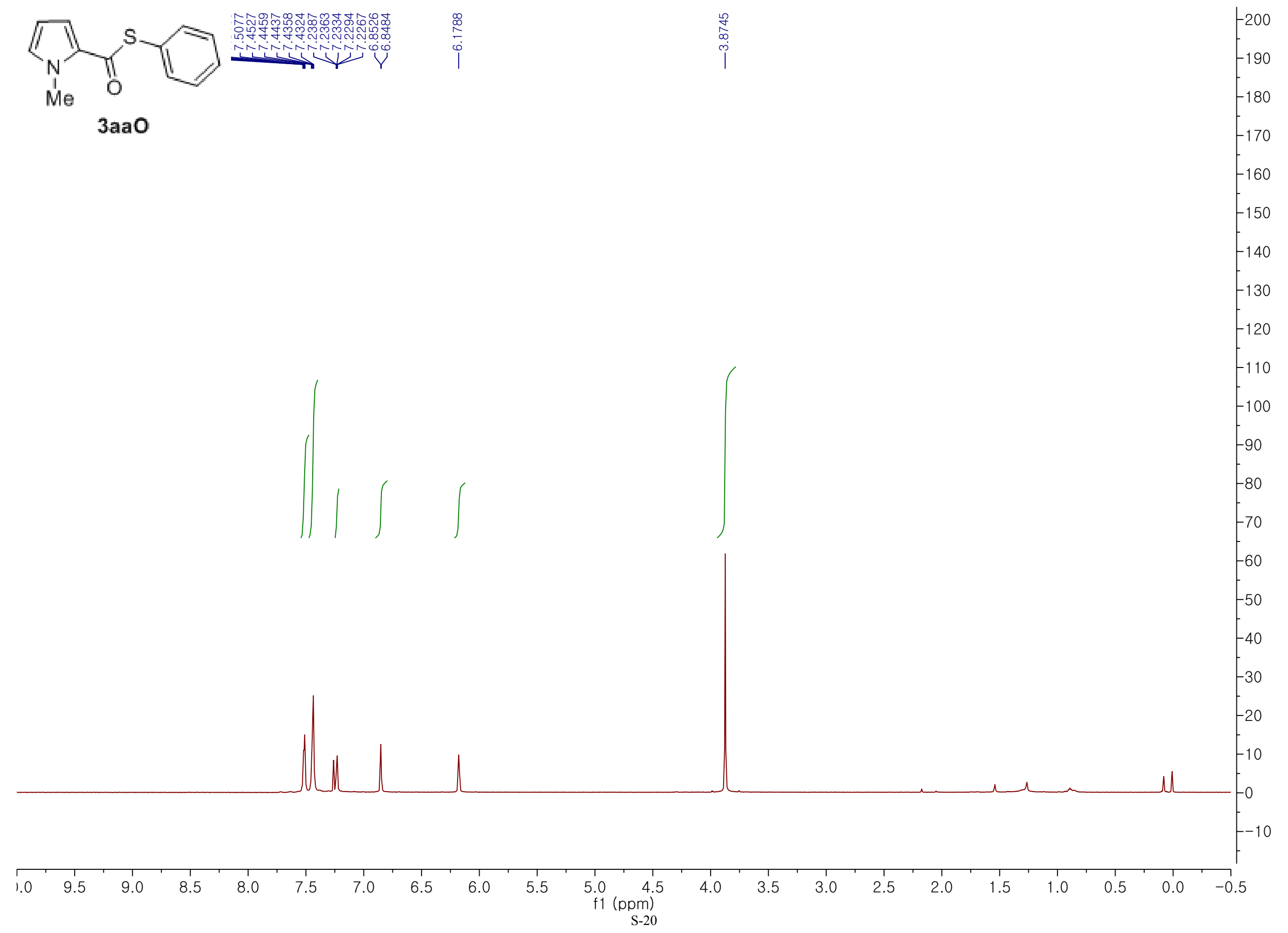




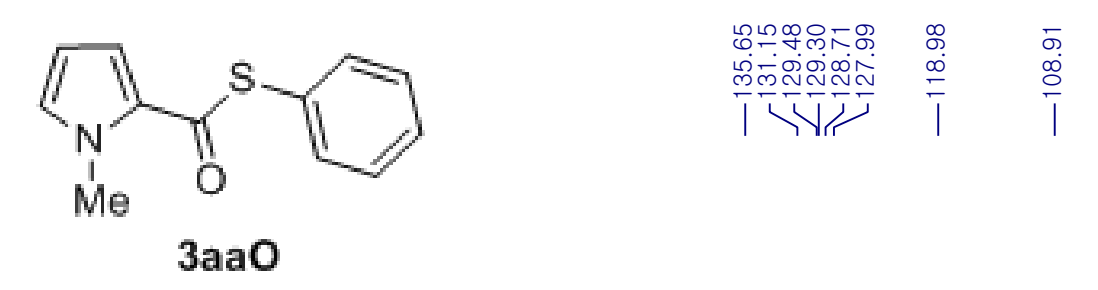

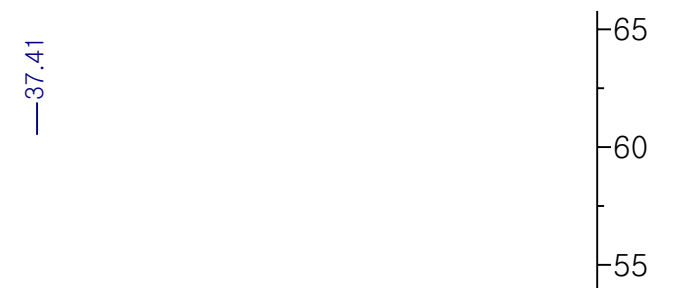

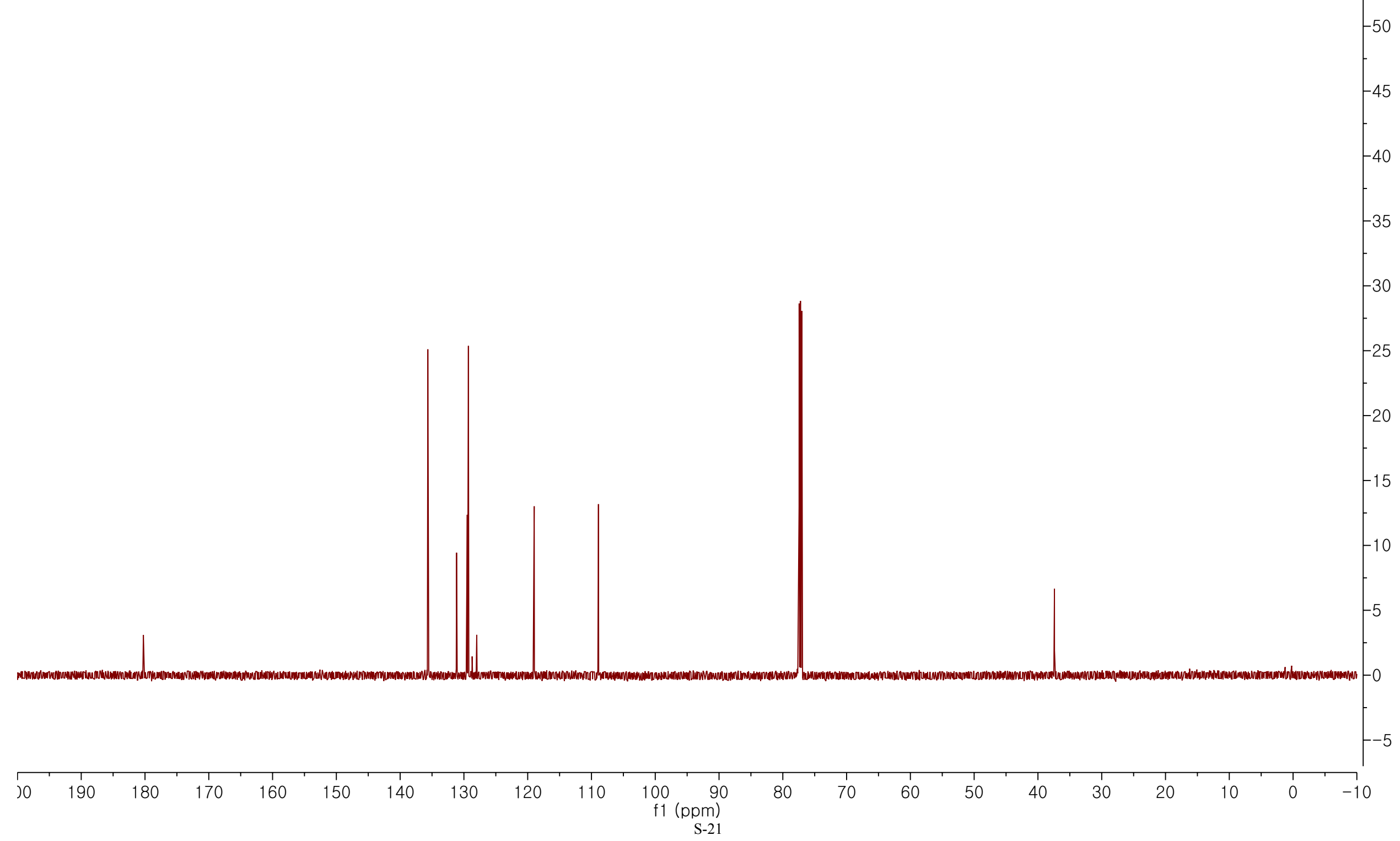




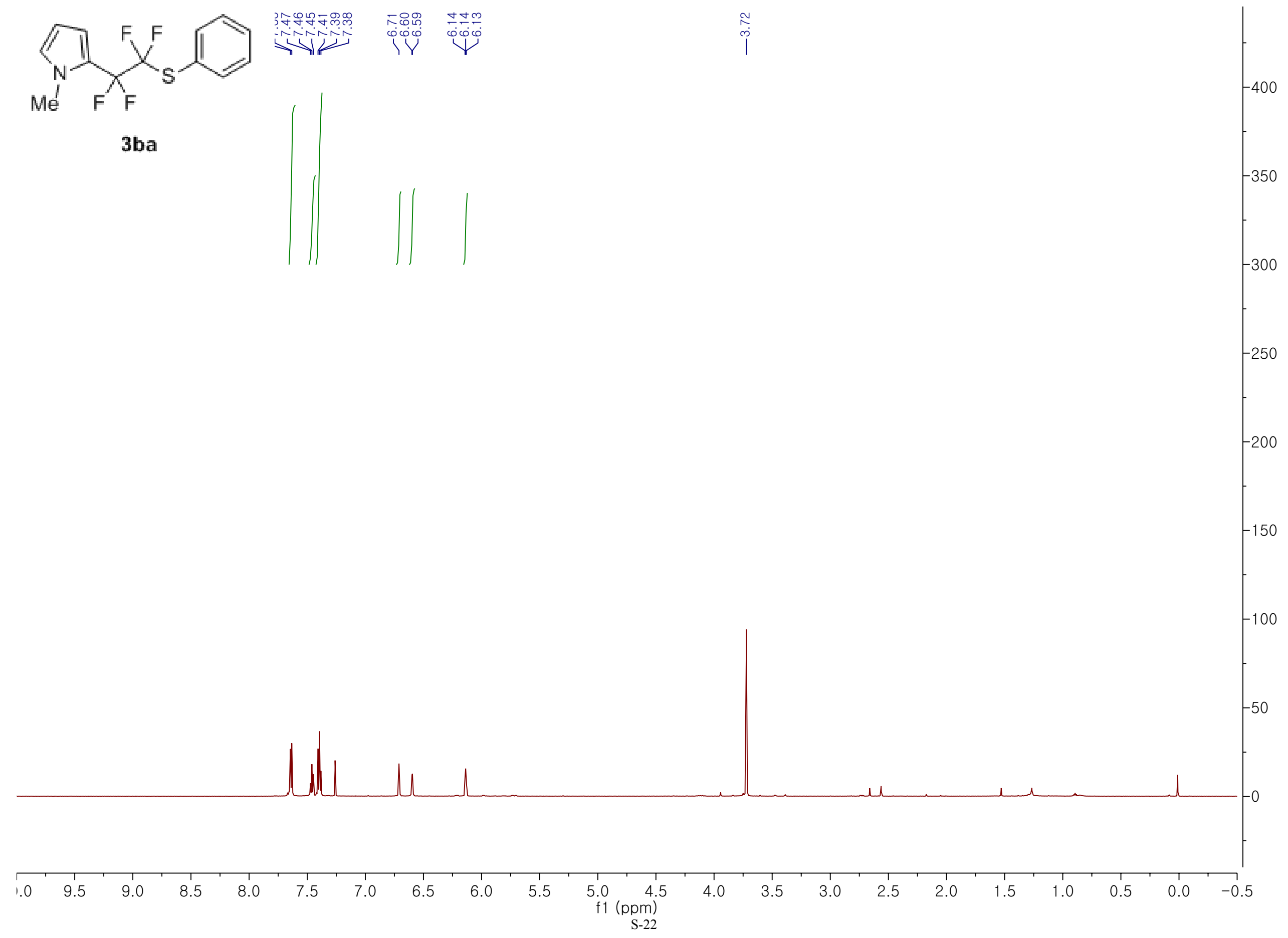




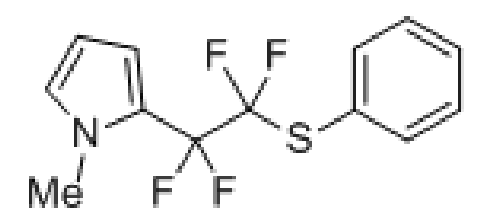

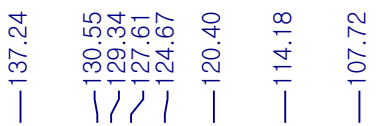

3 ba

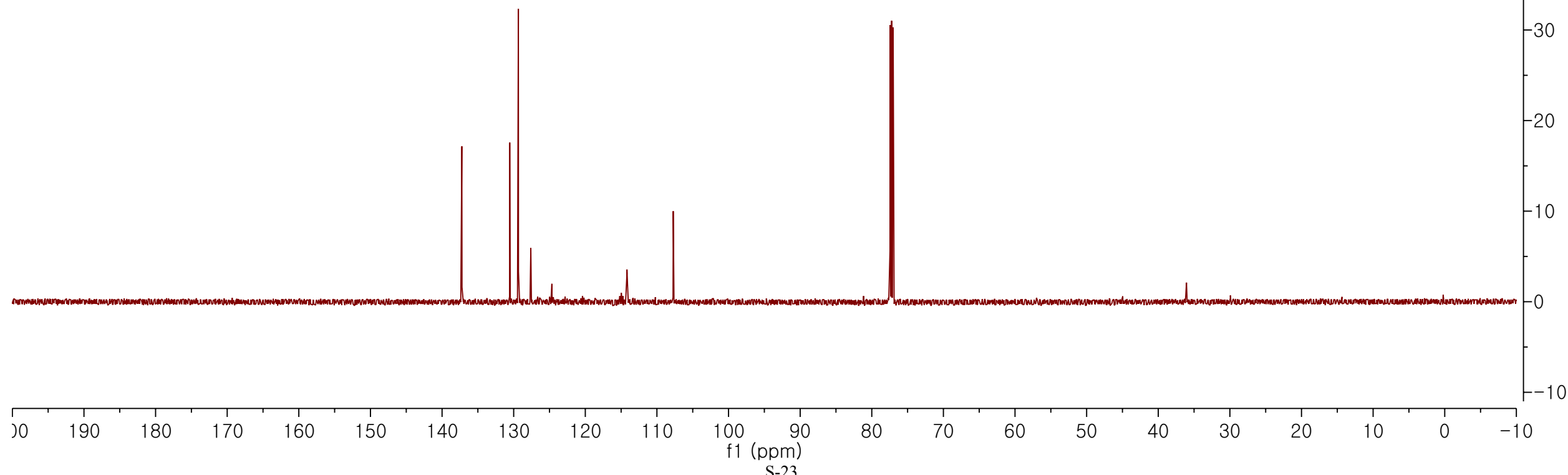




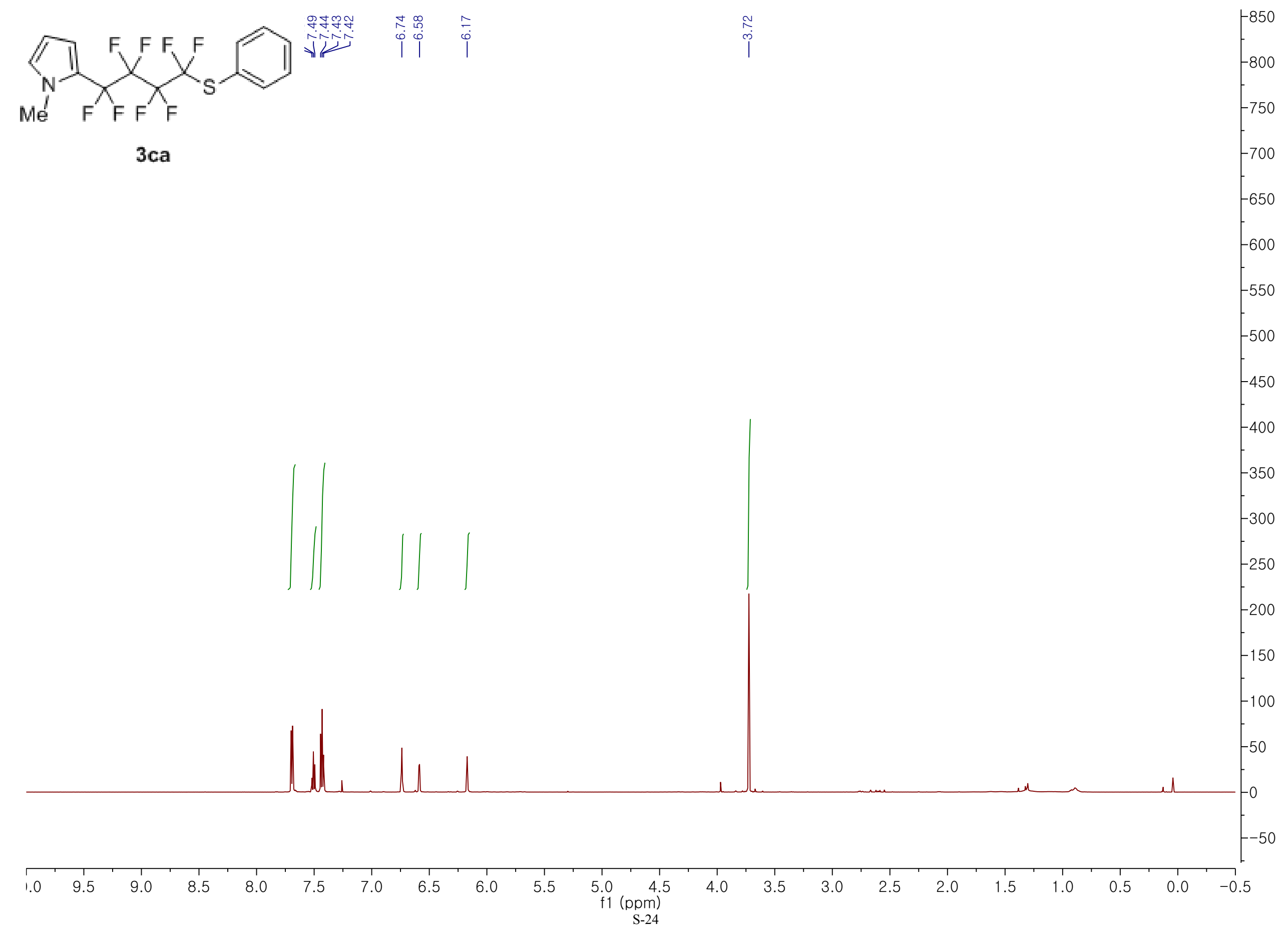




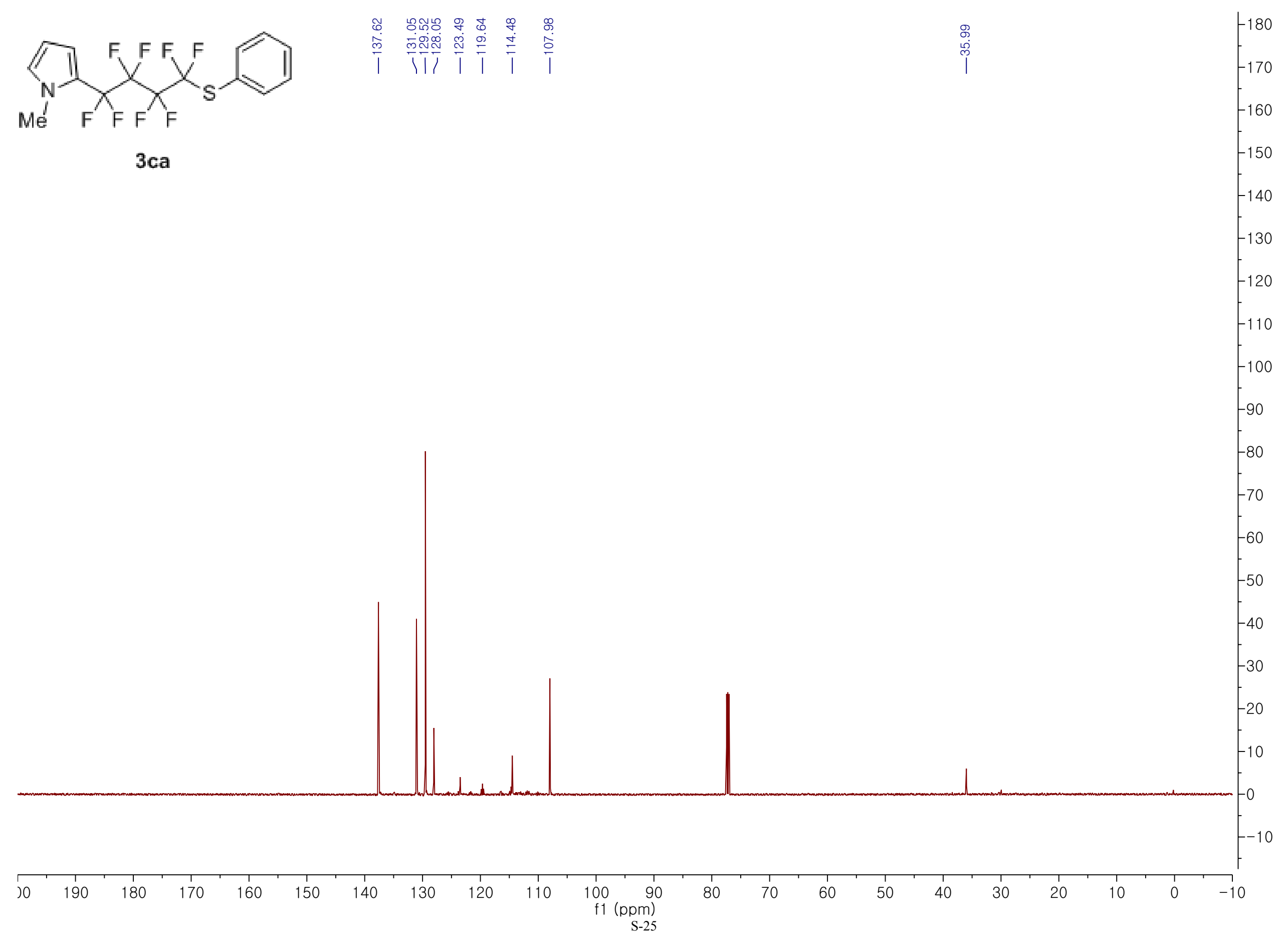




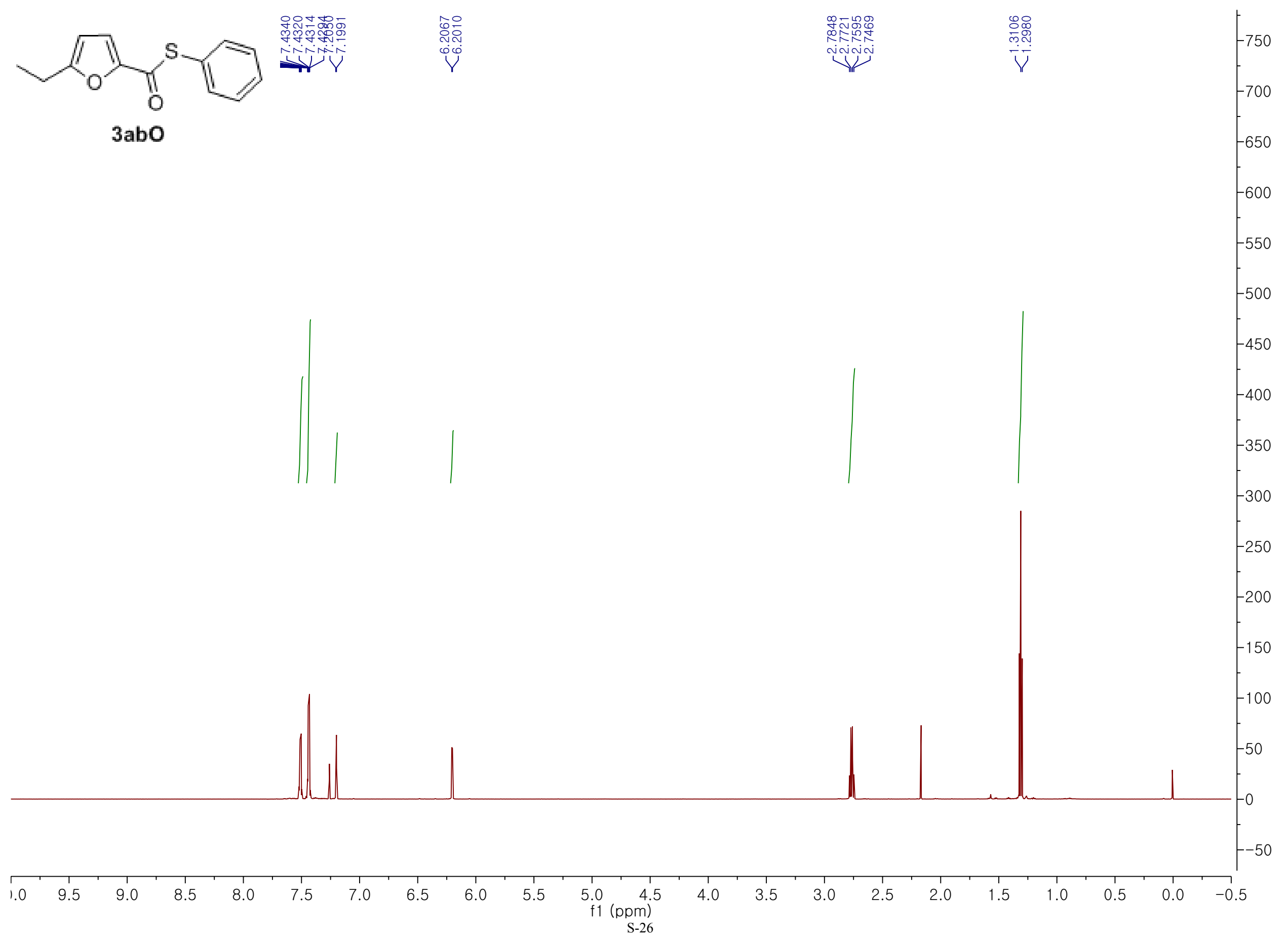




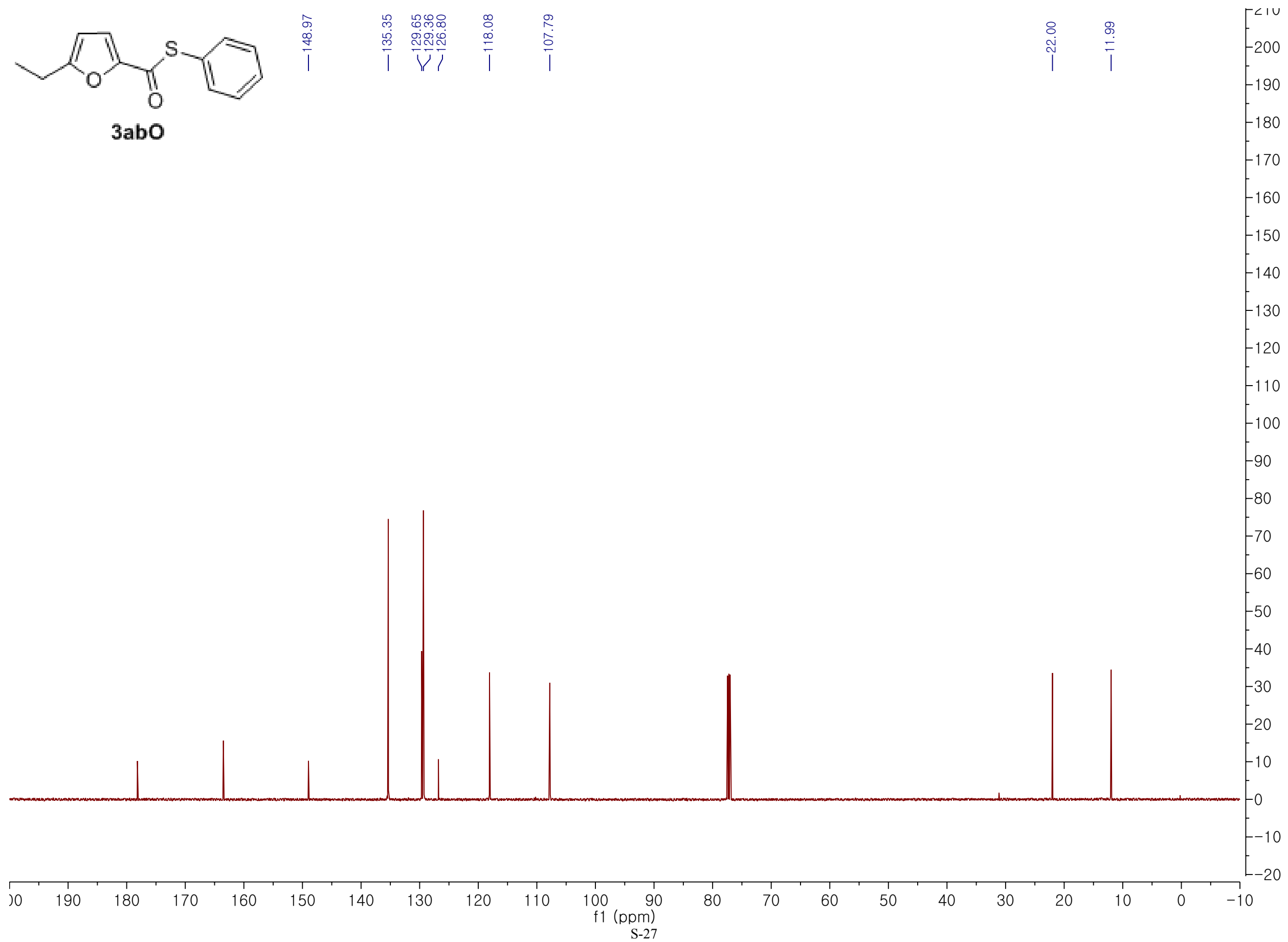




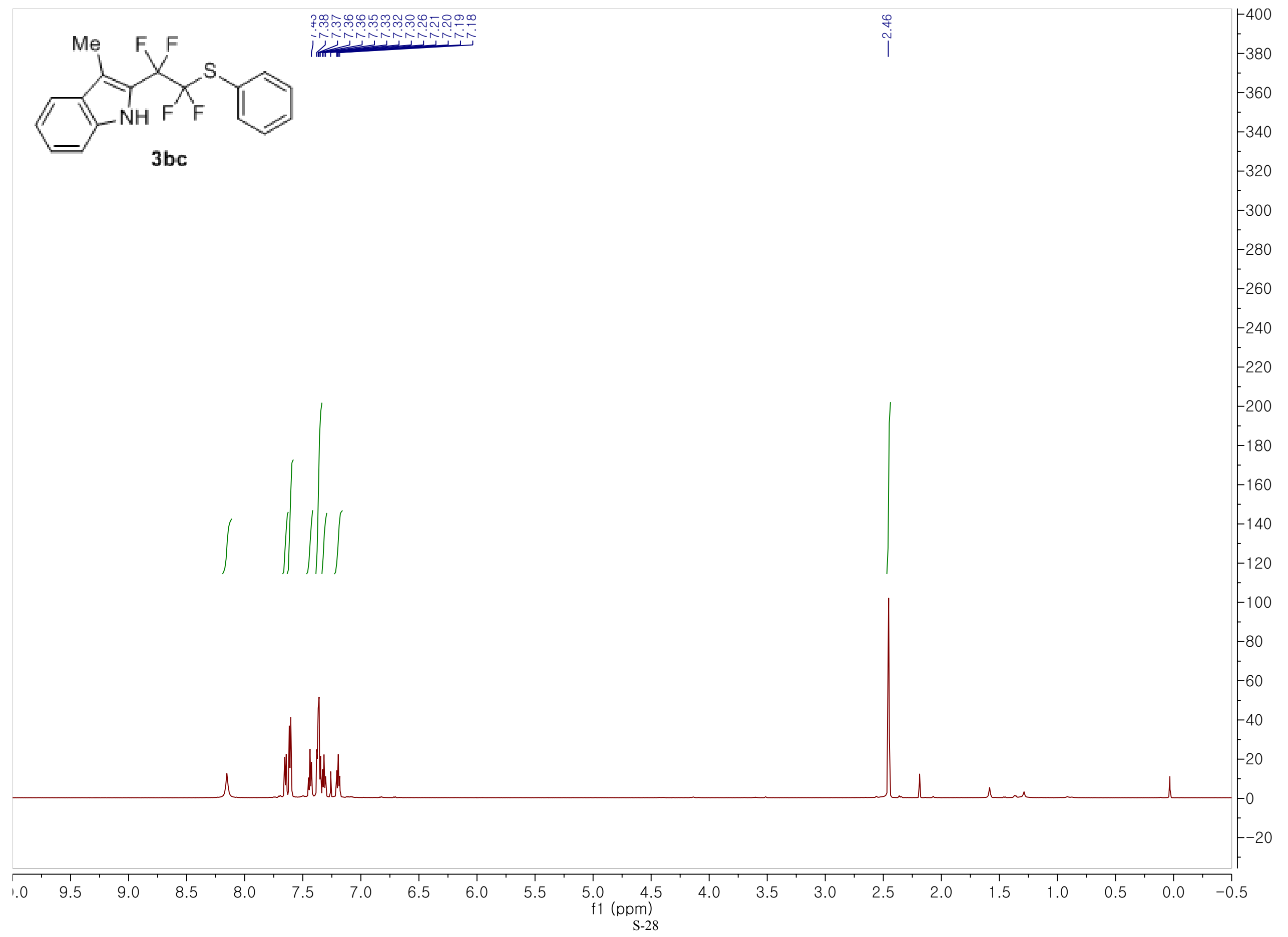




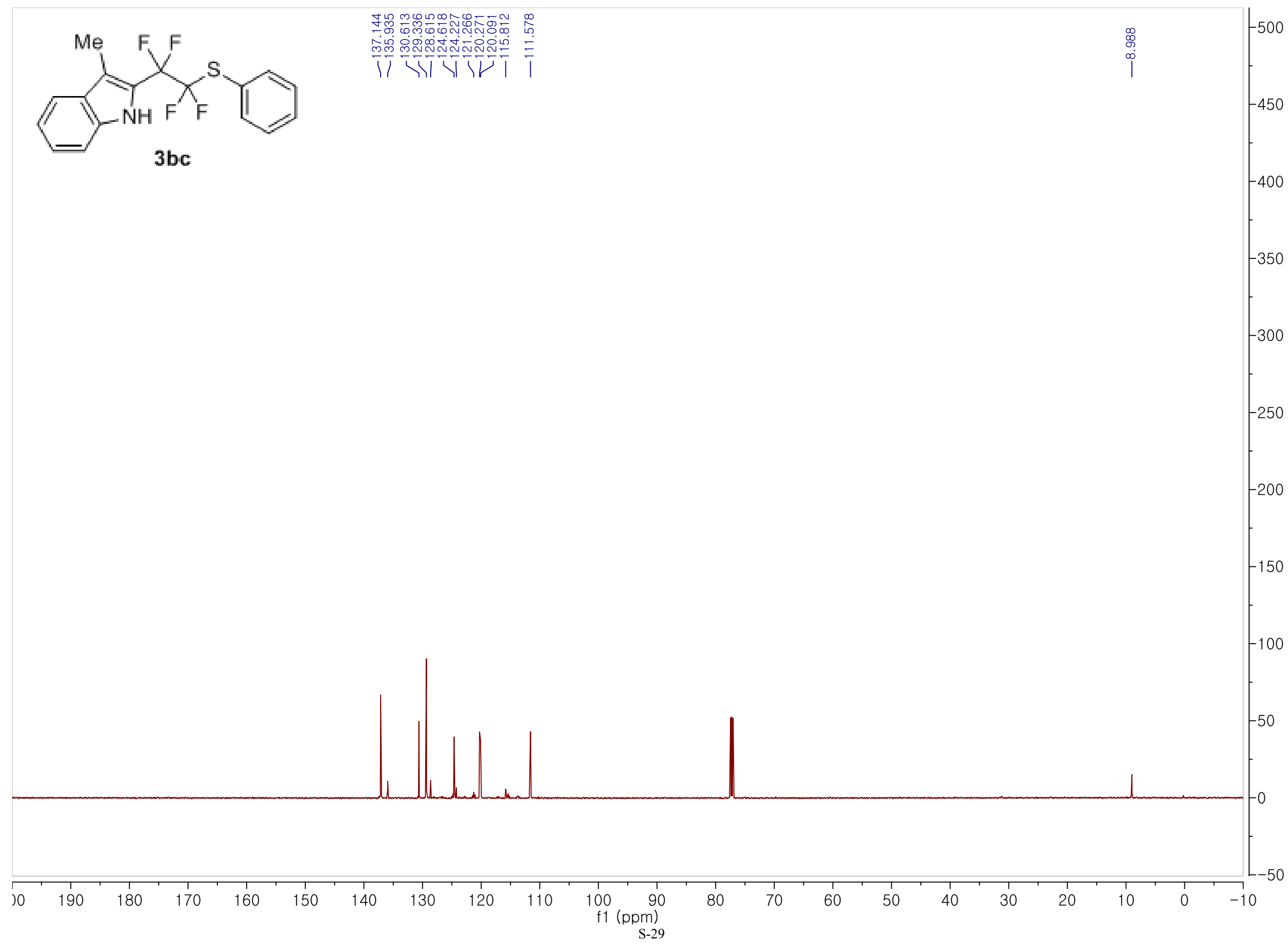




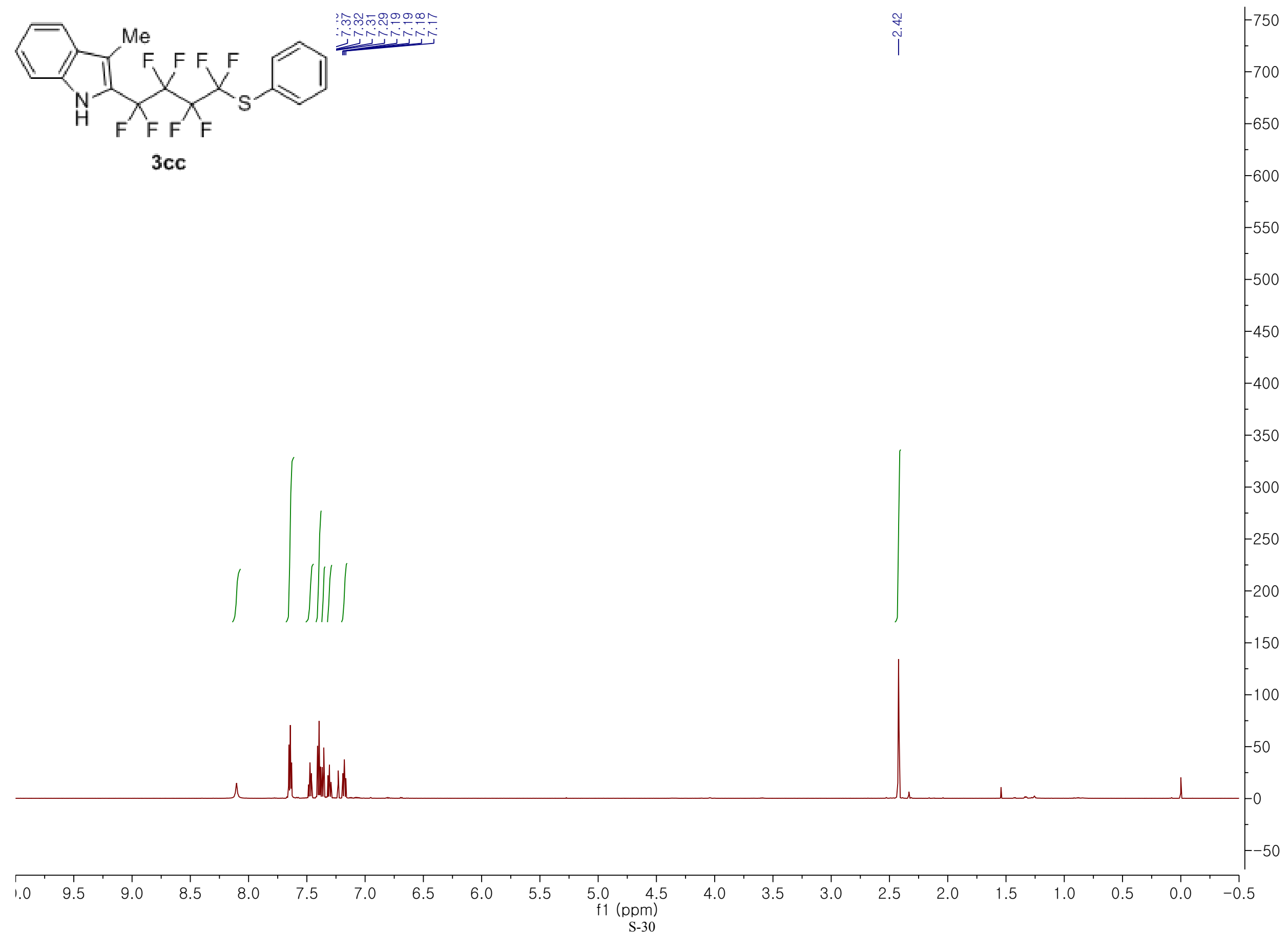




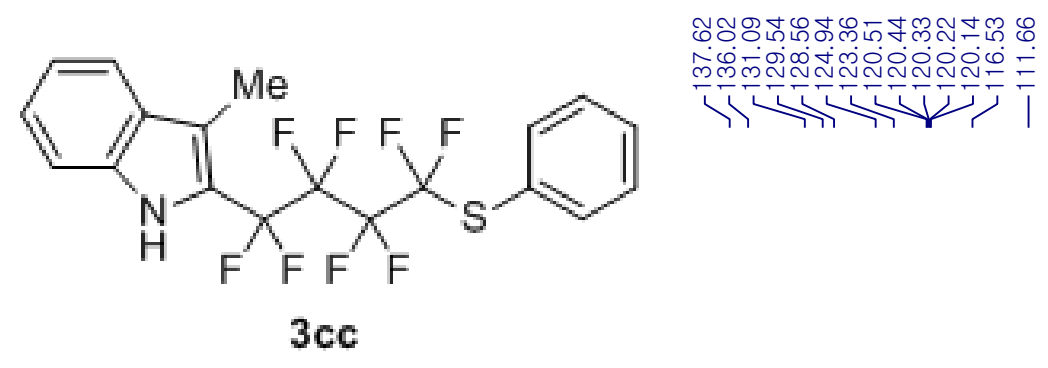

do.

疗

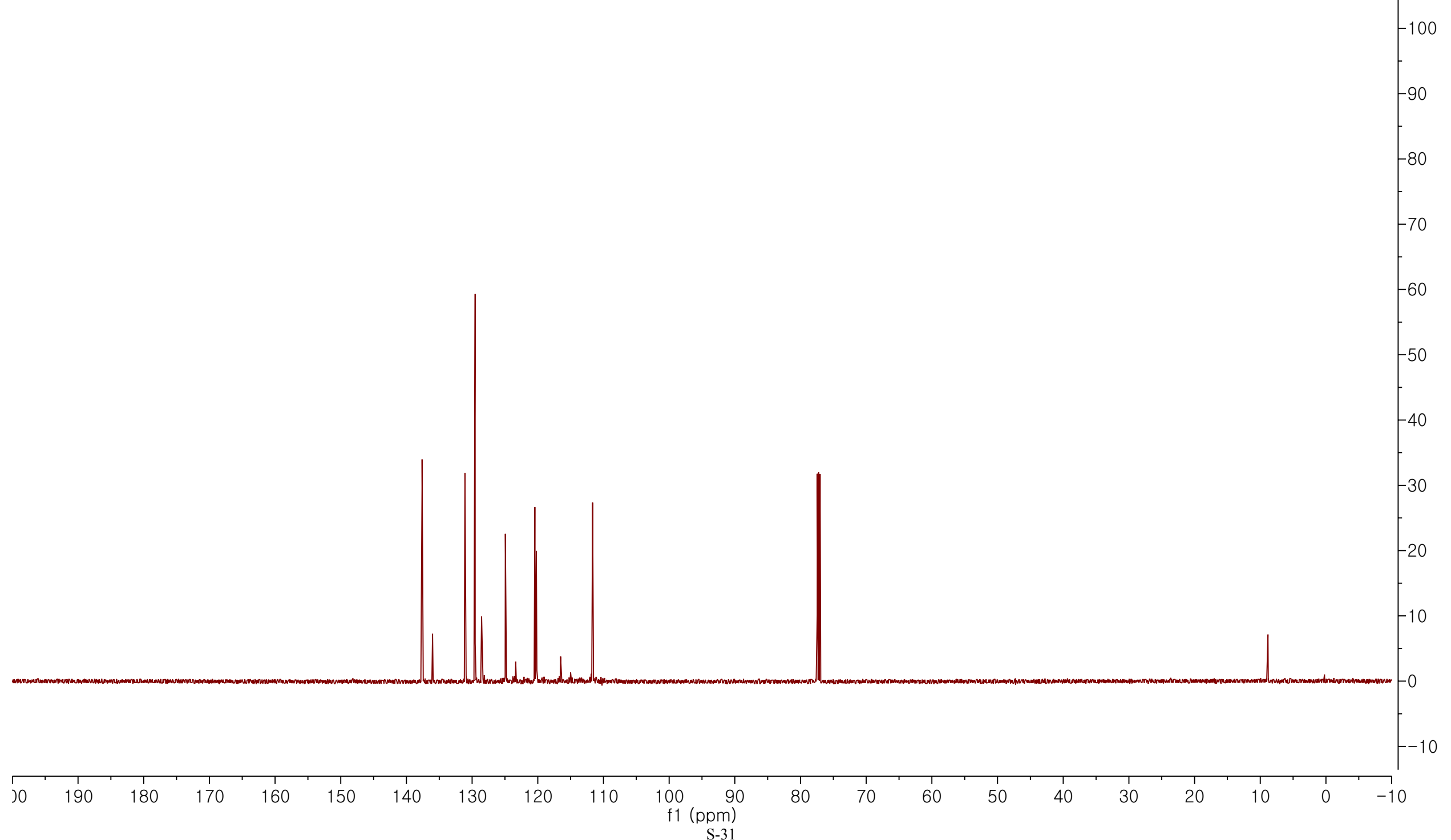




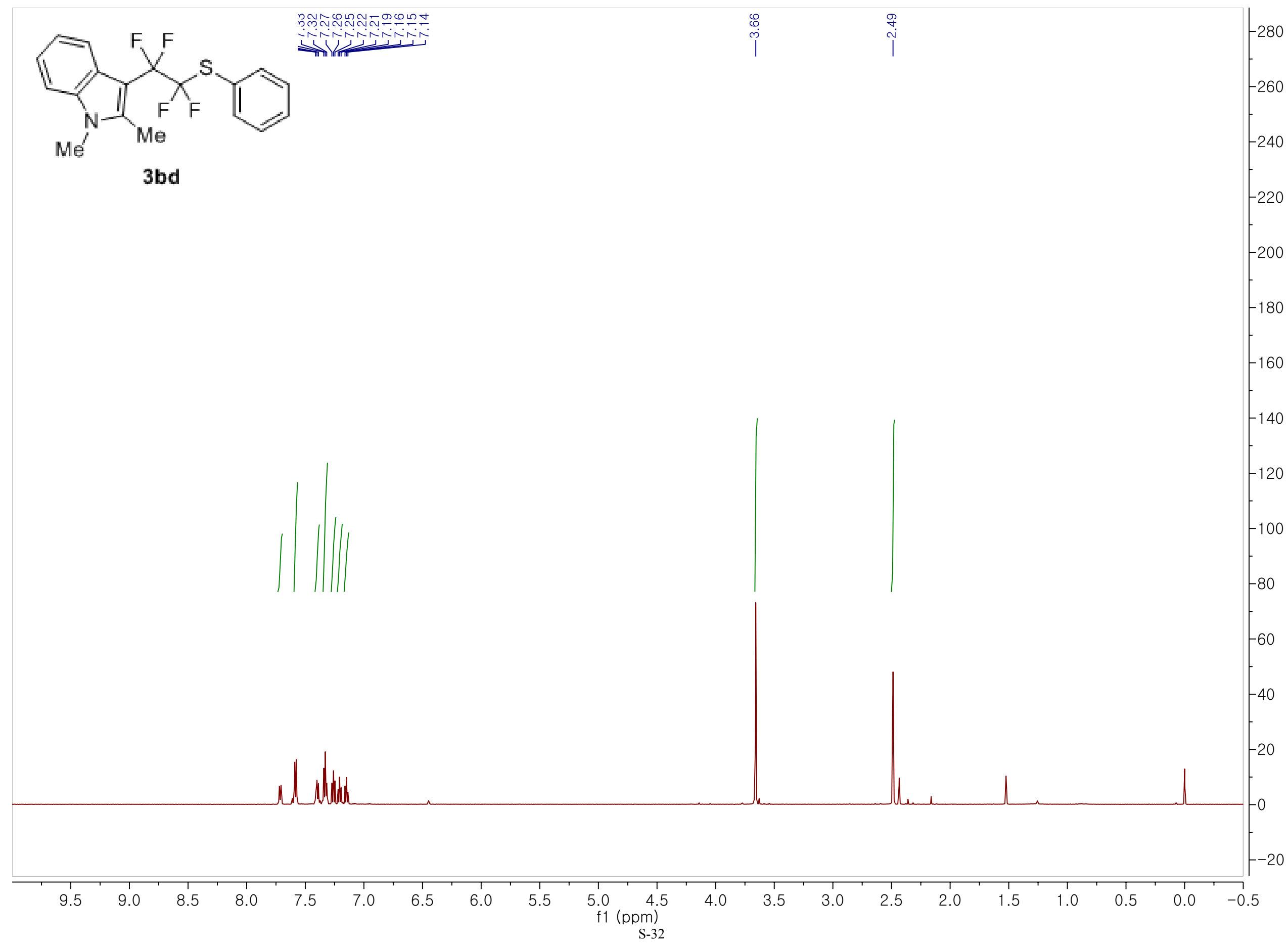




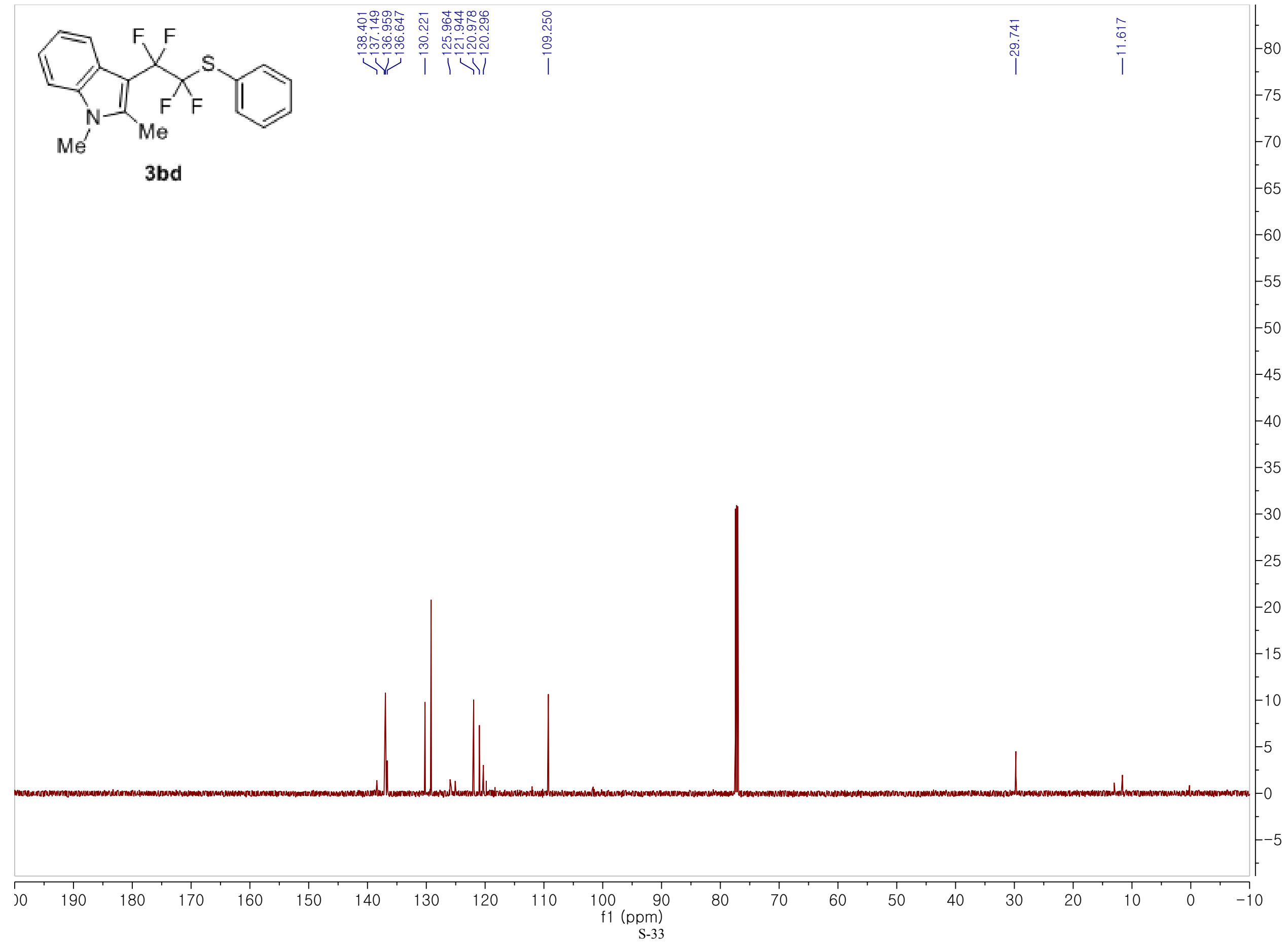




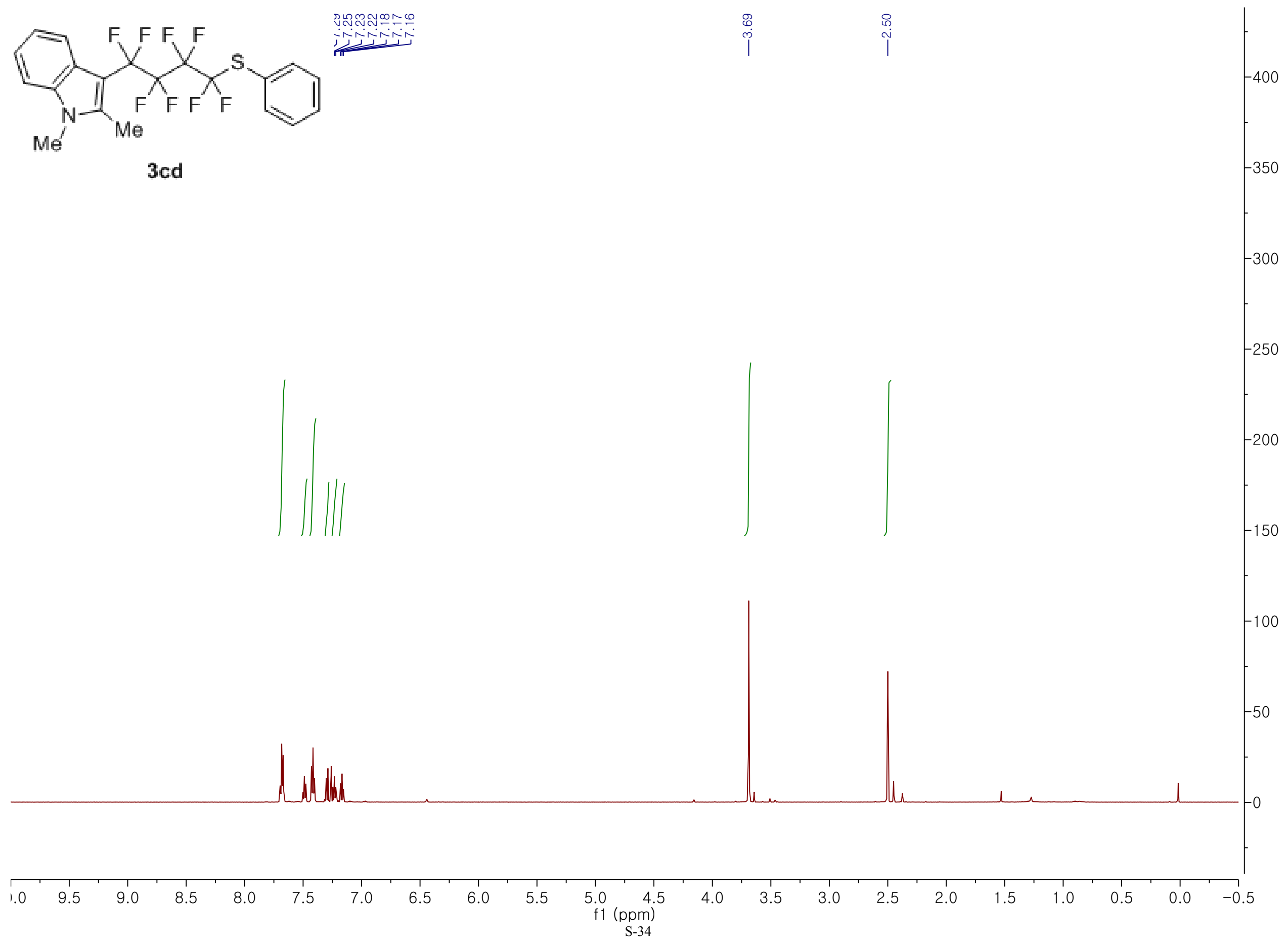




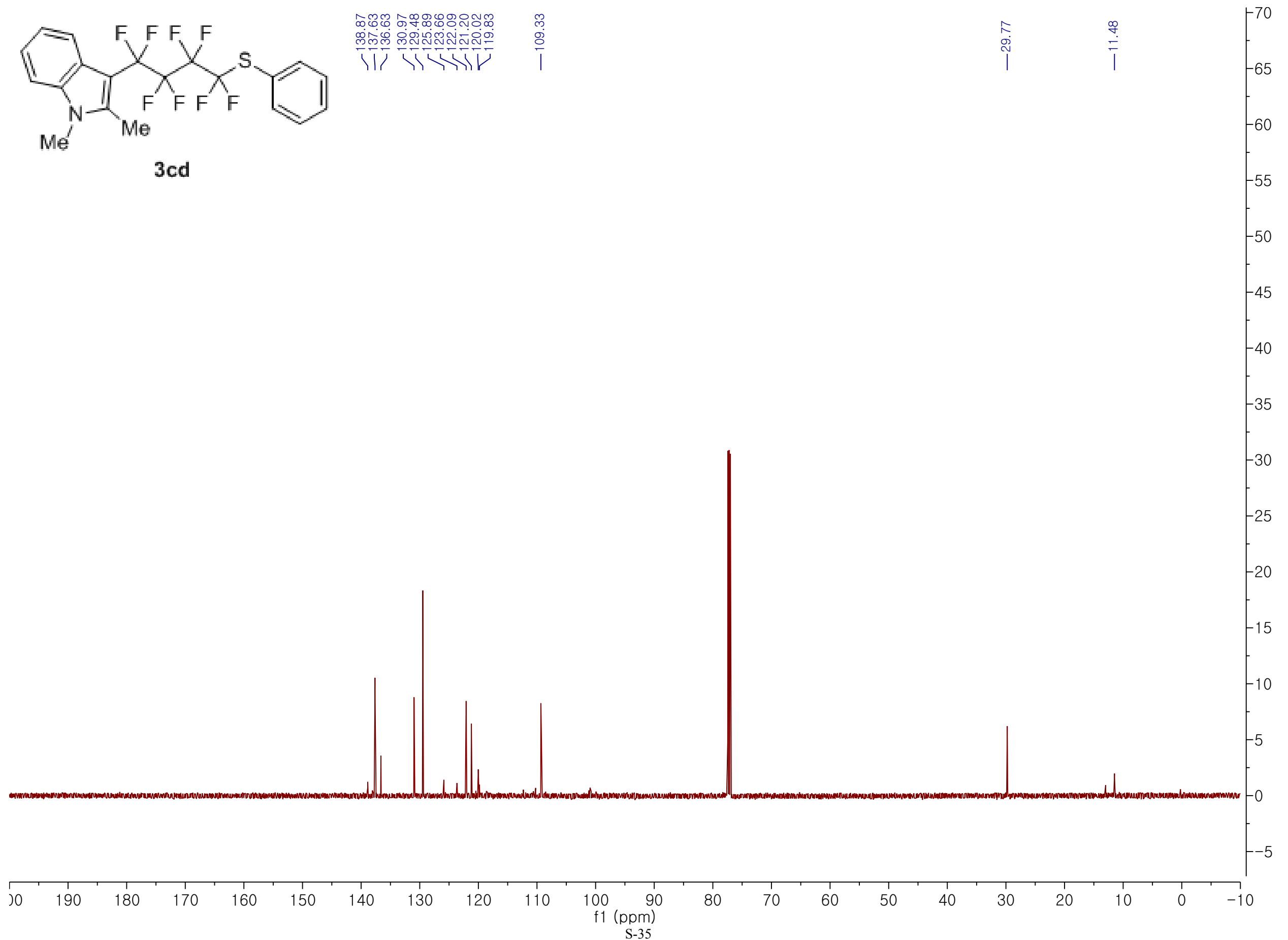




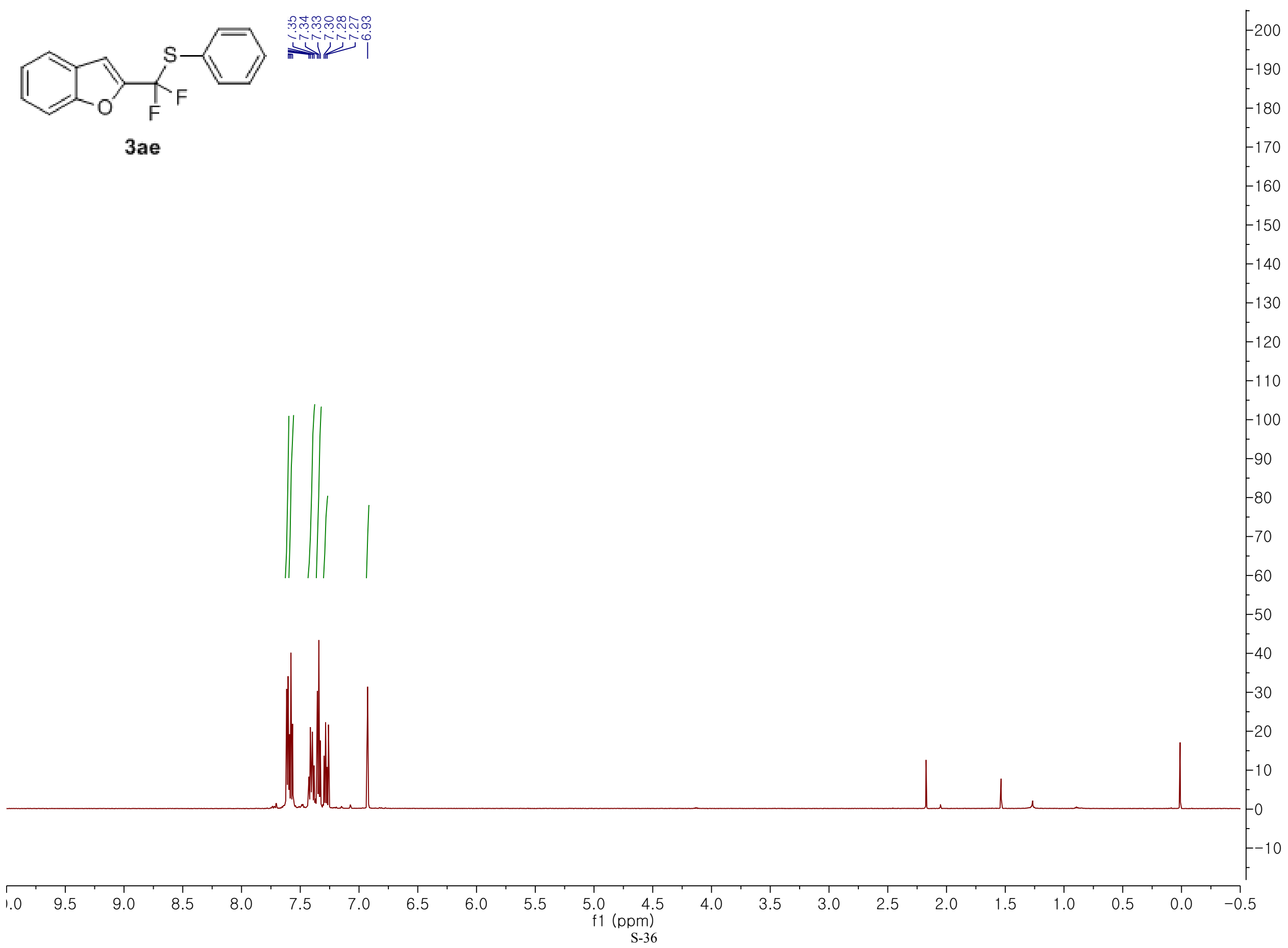




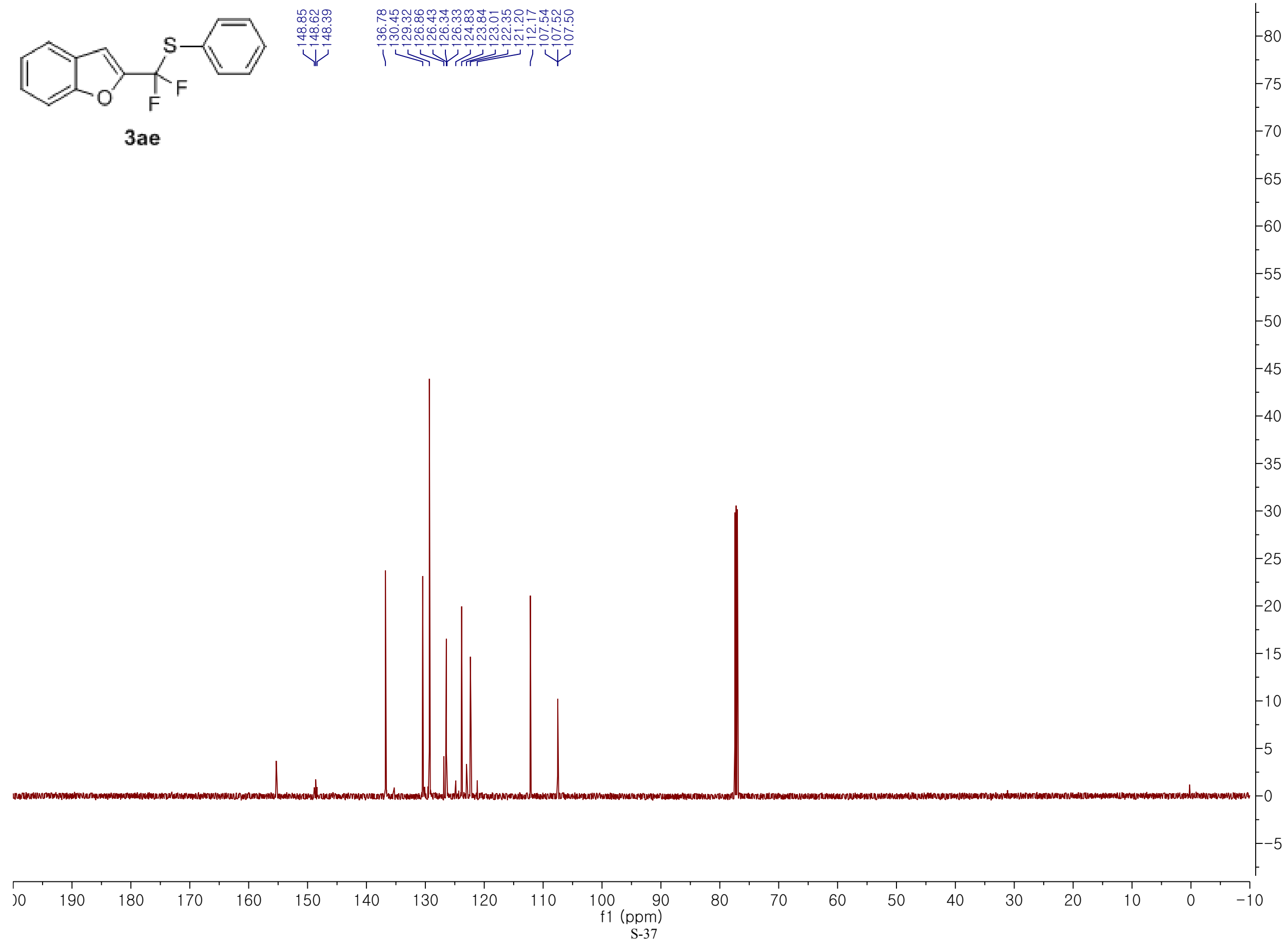




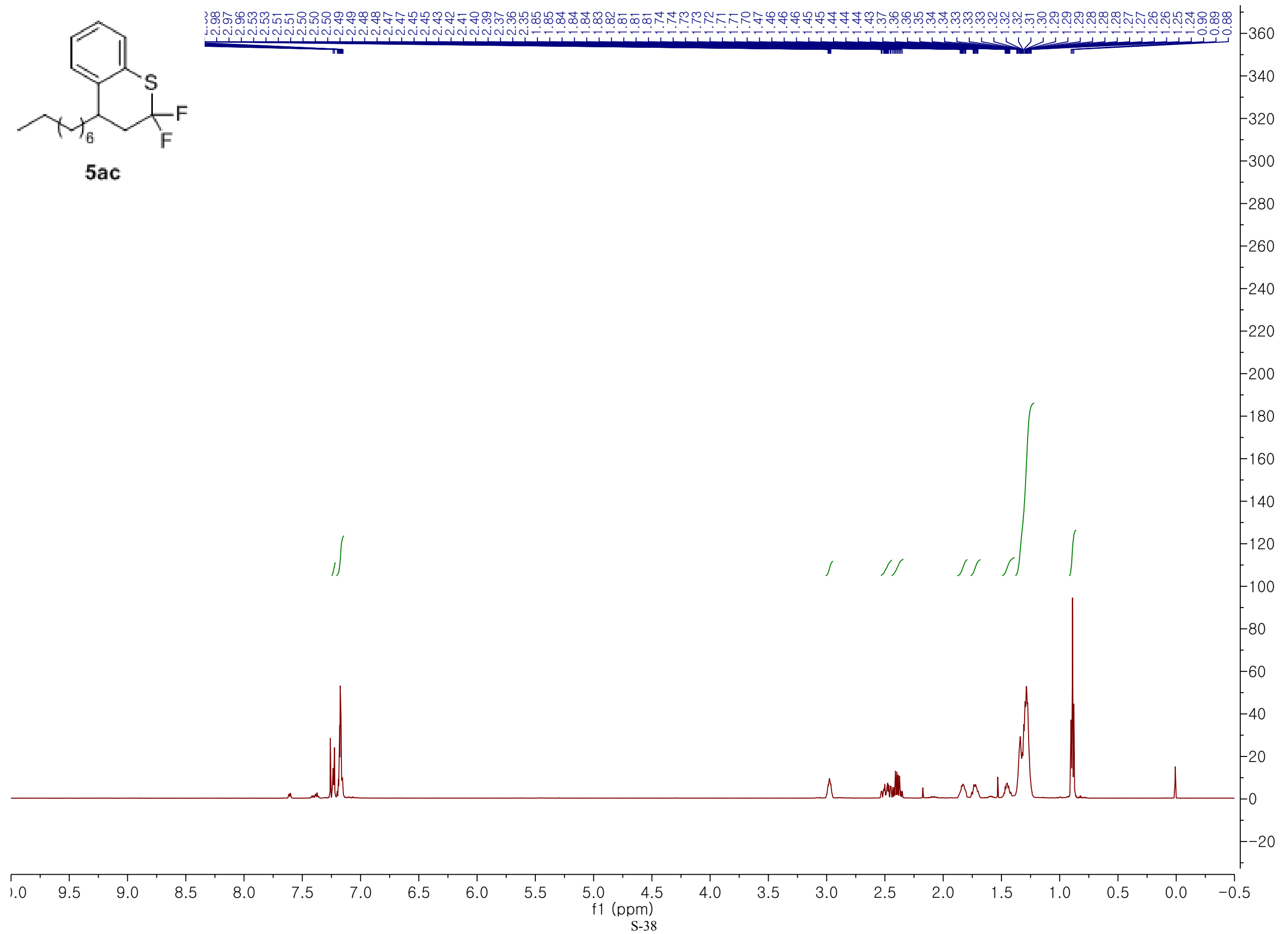



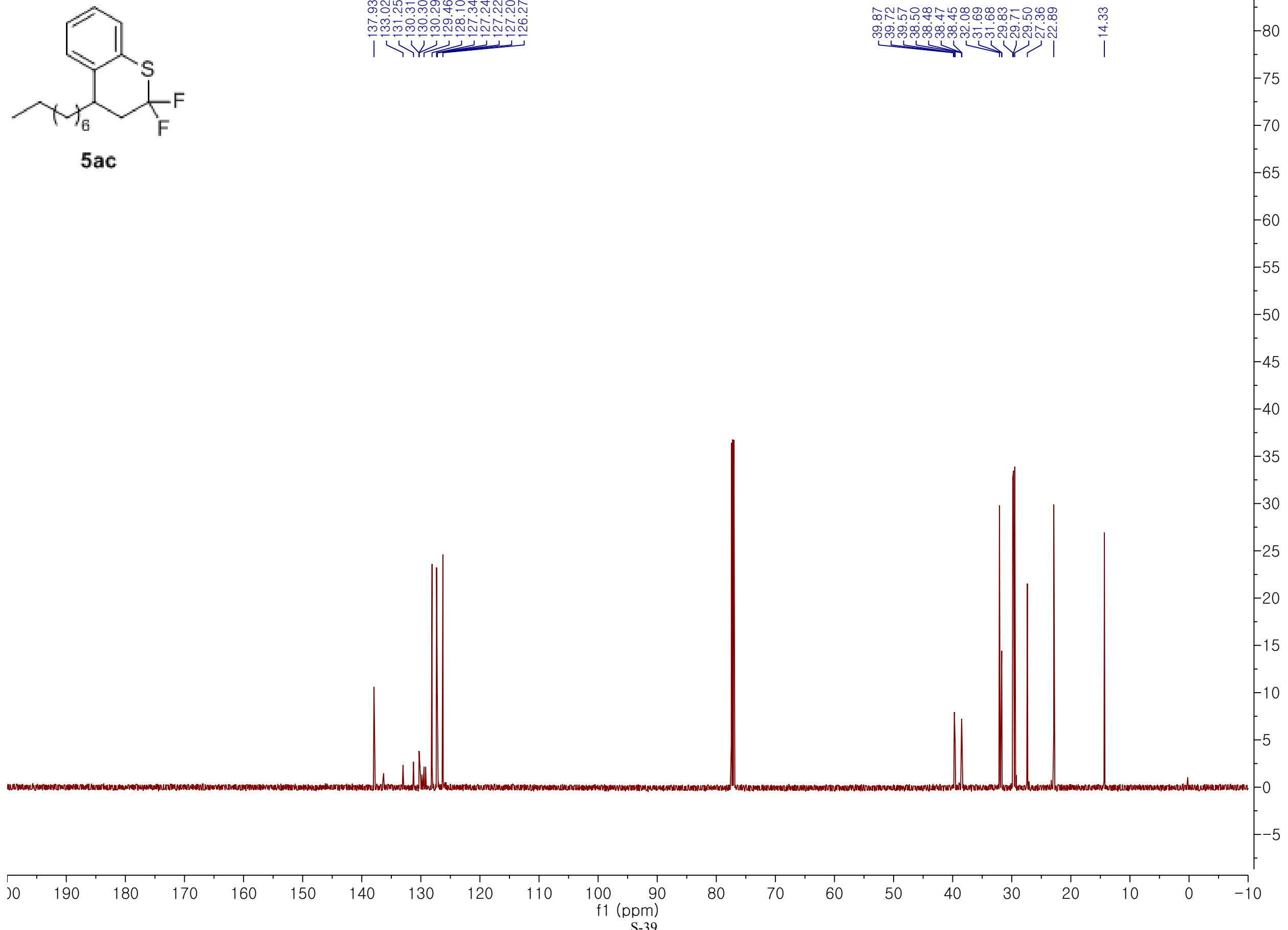


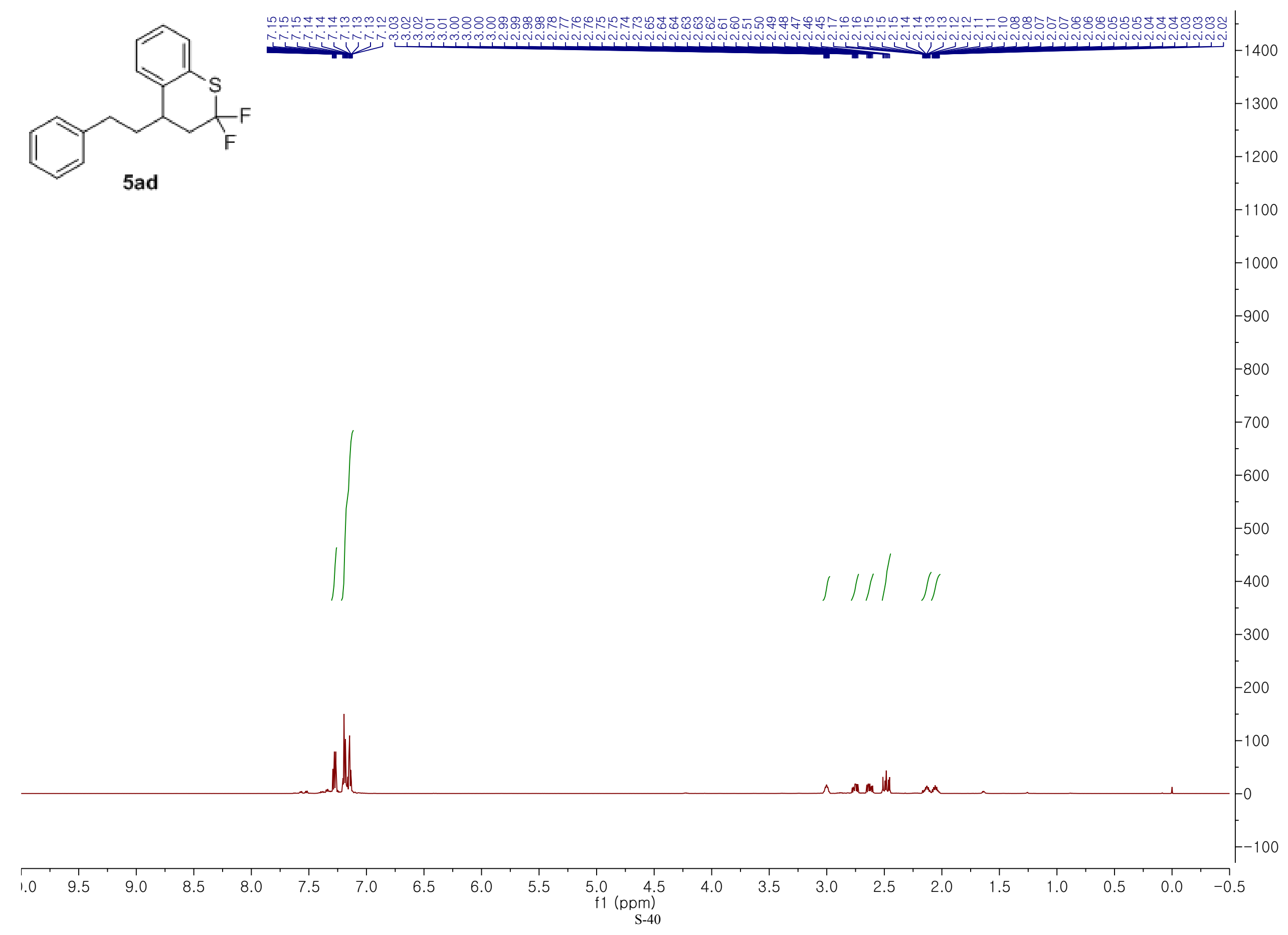




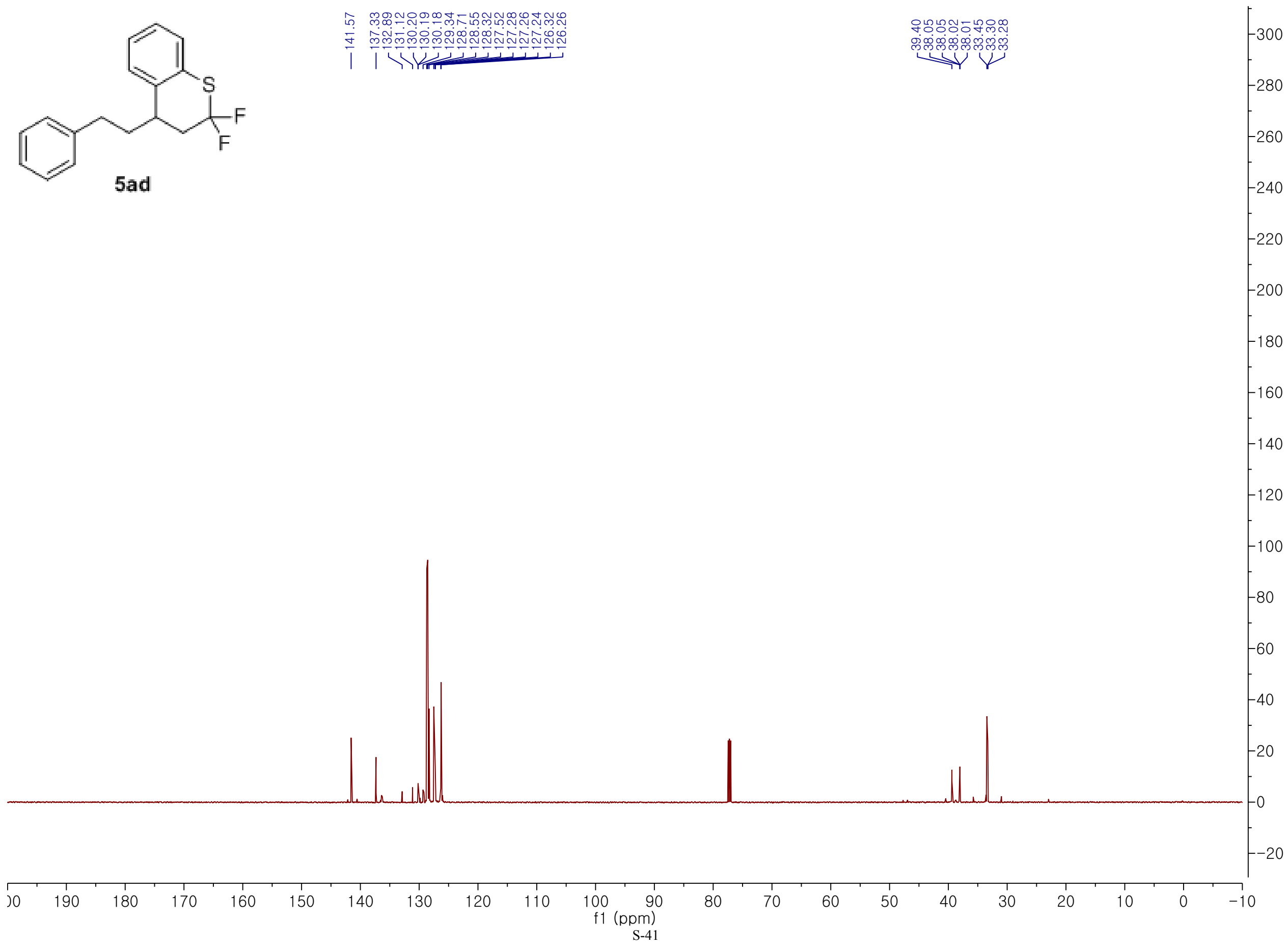




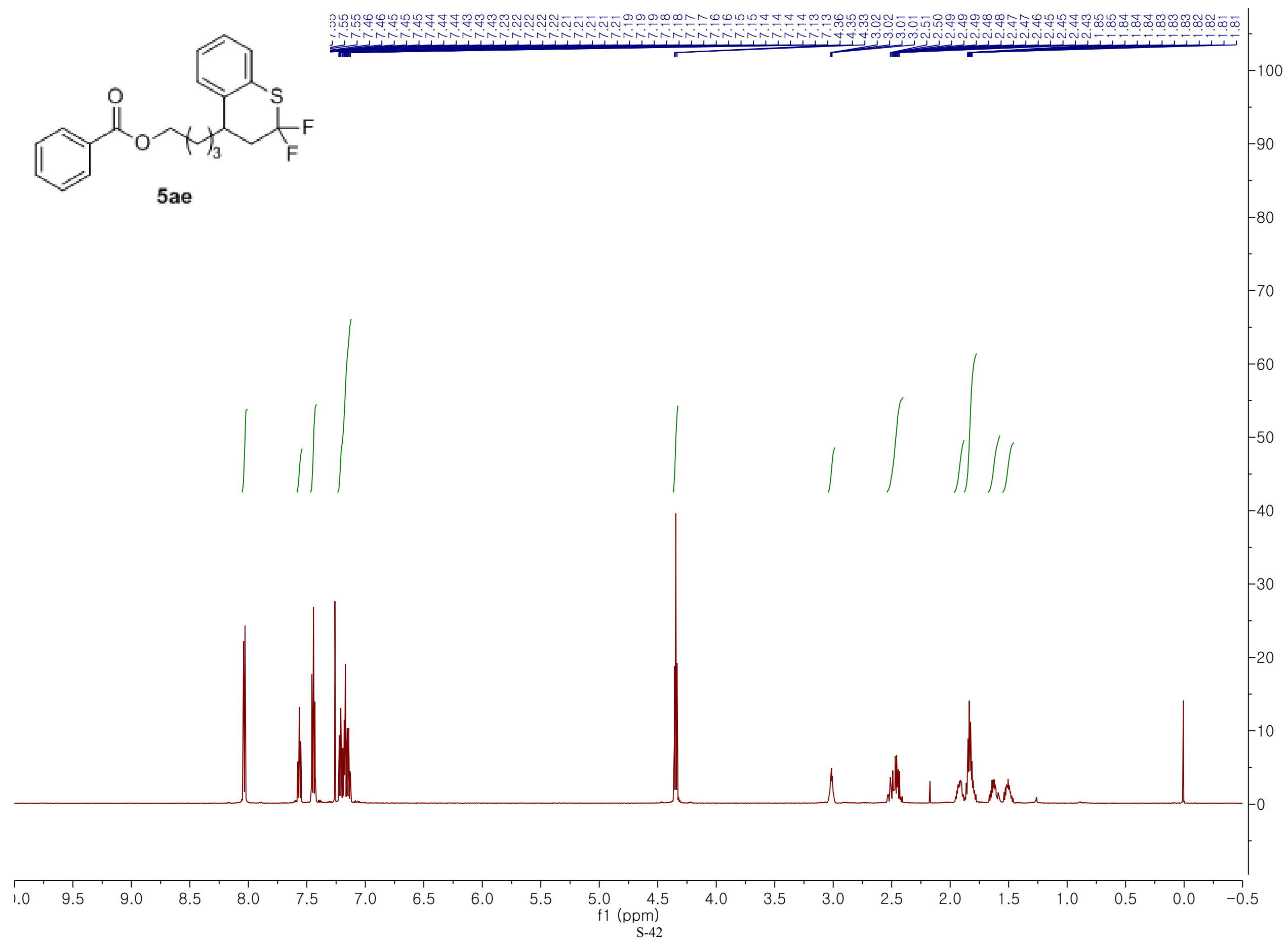




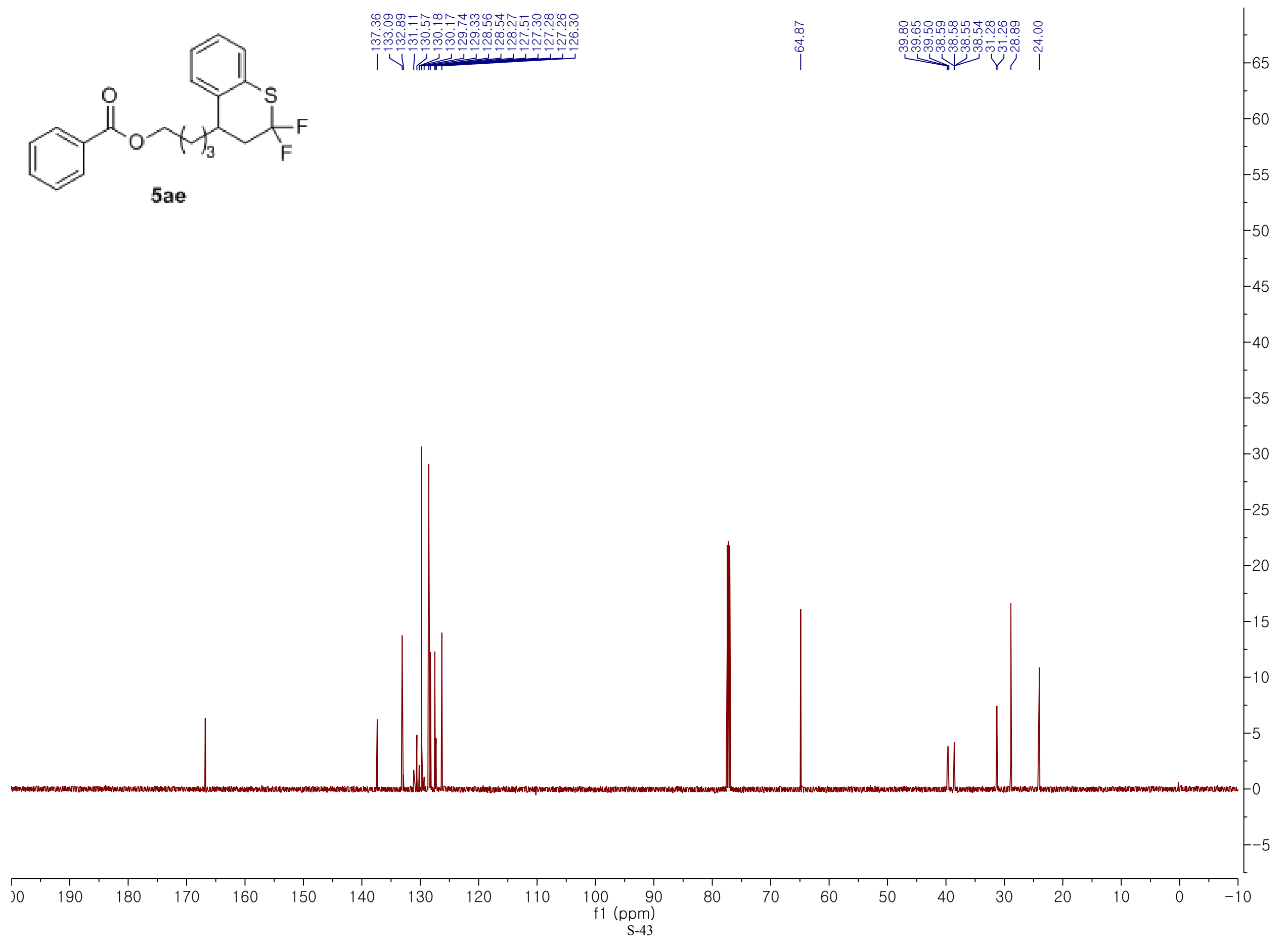




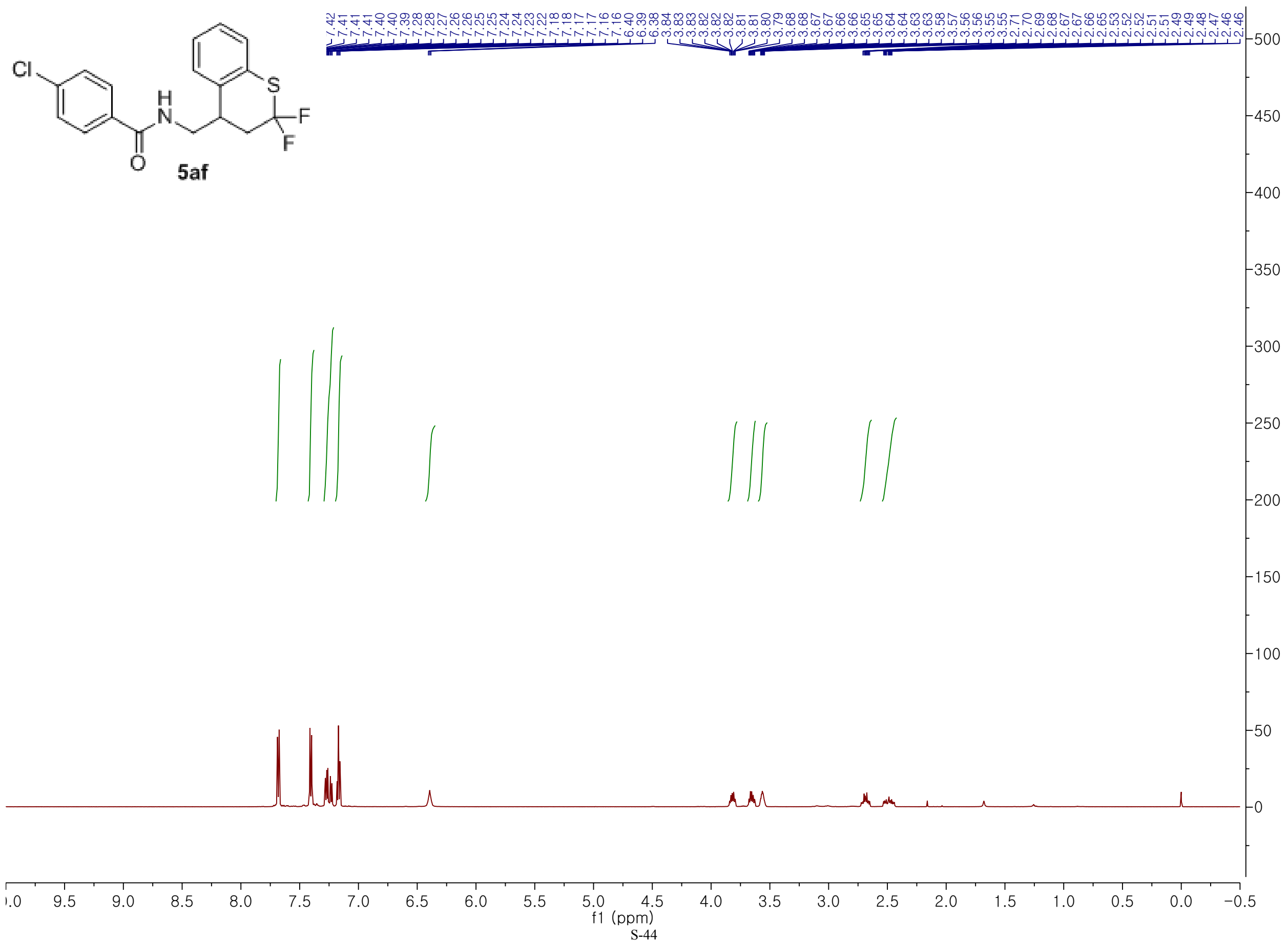



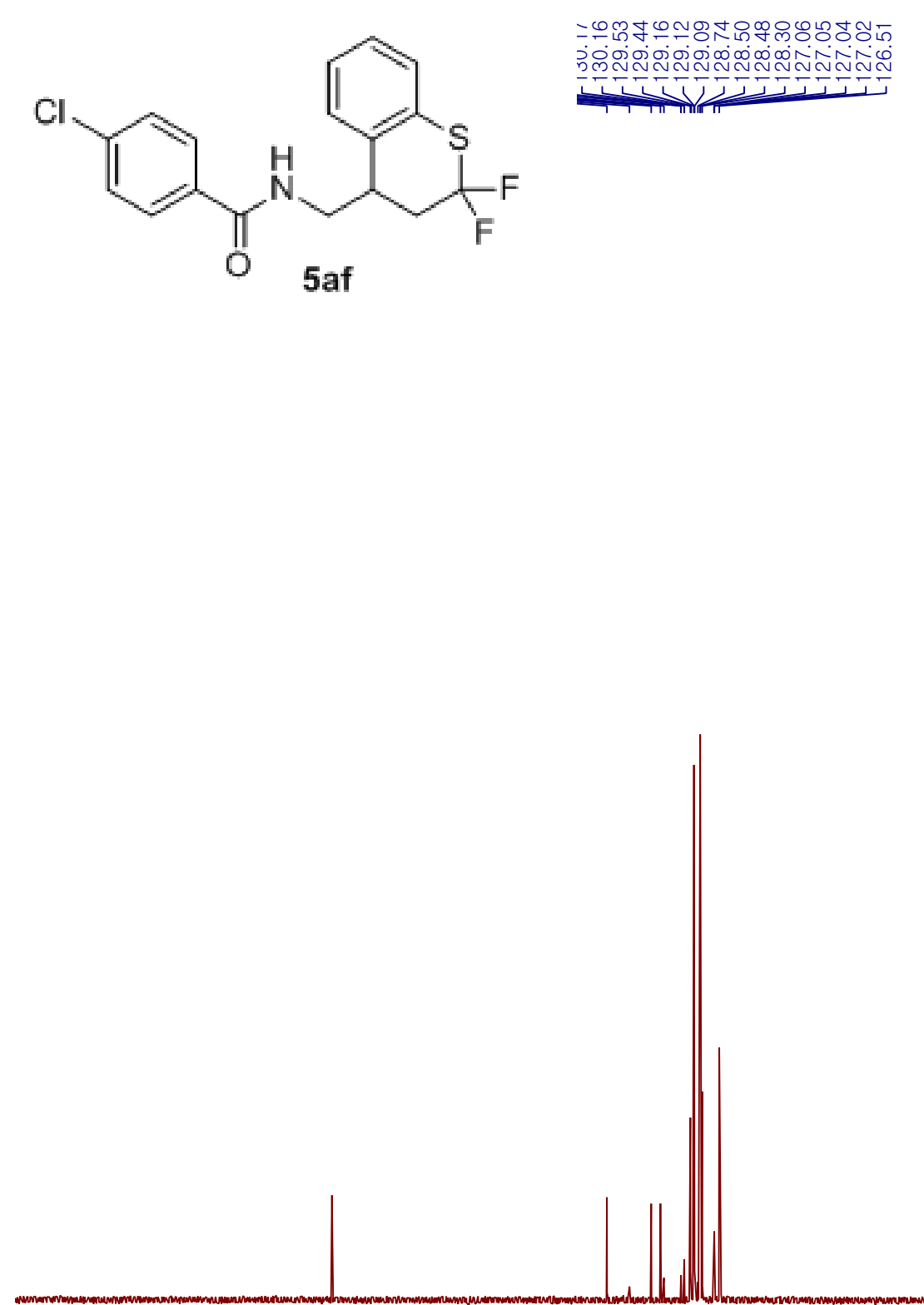


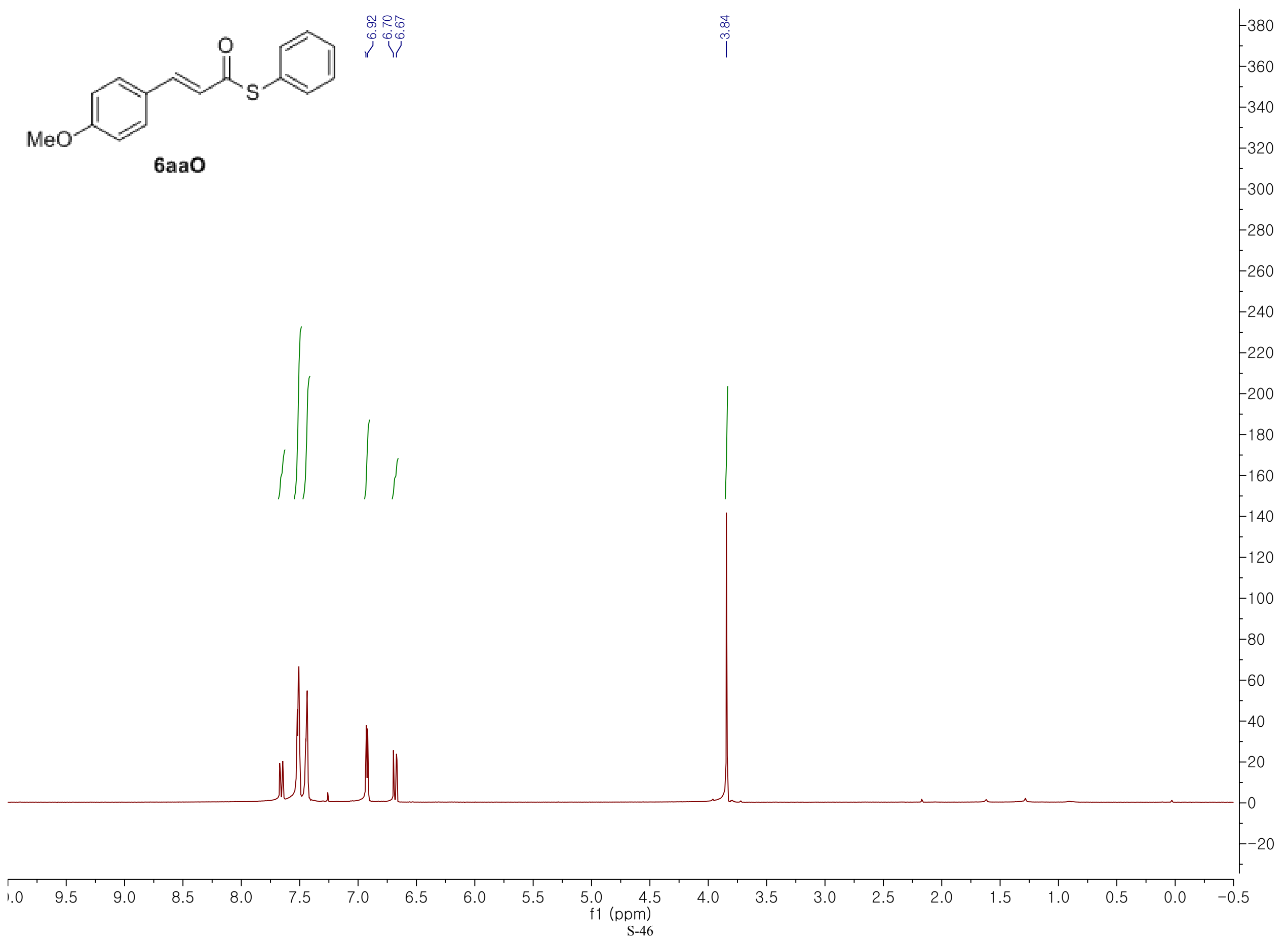




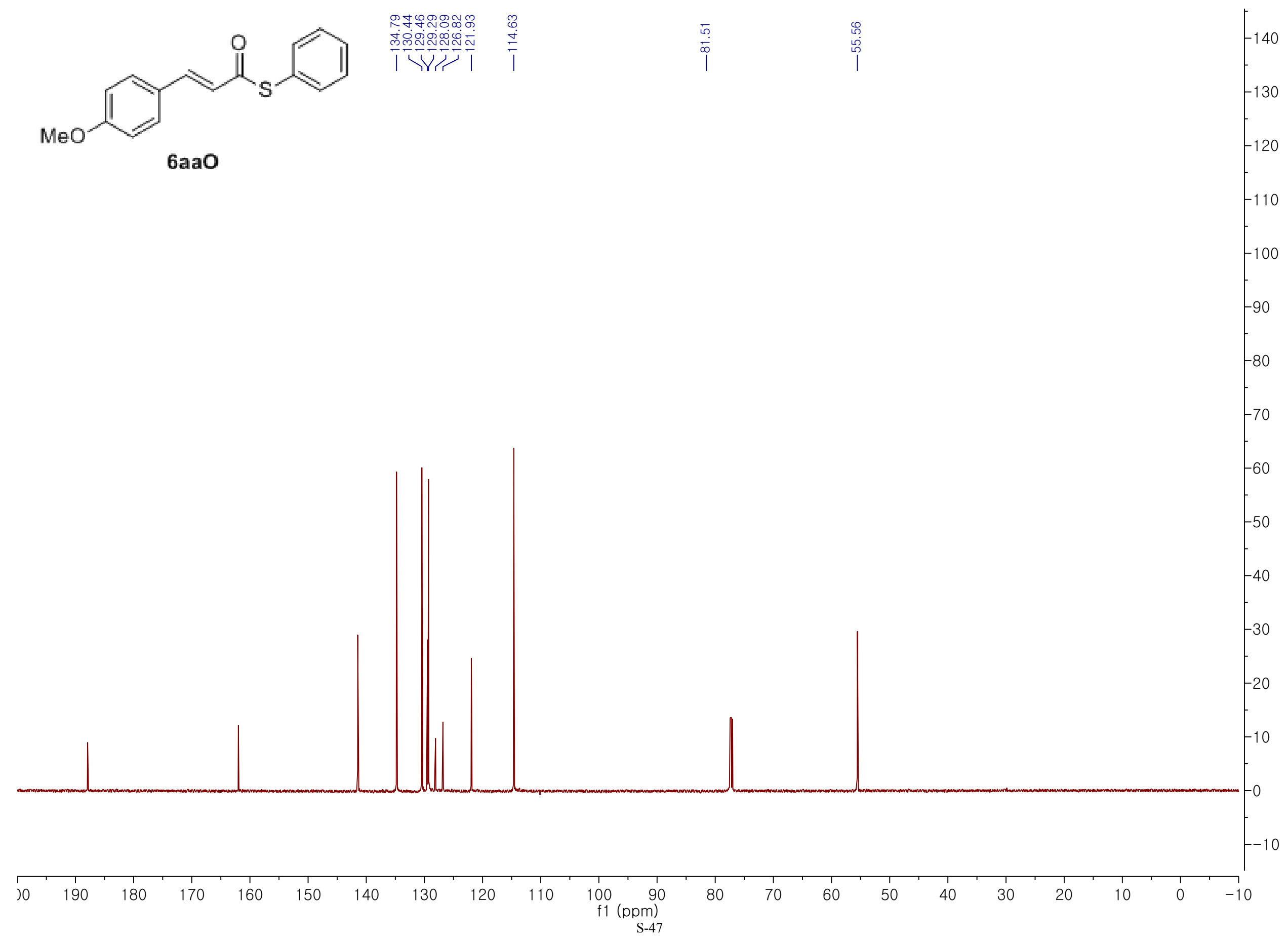




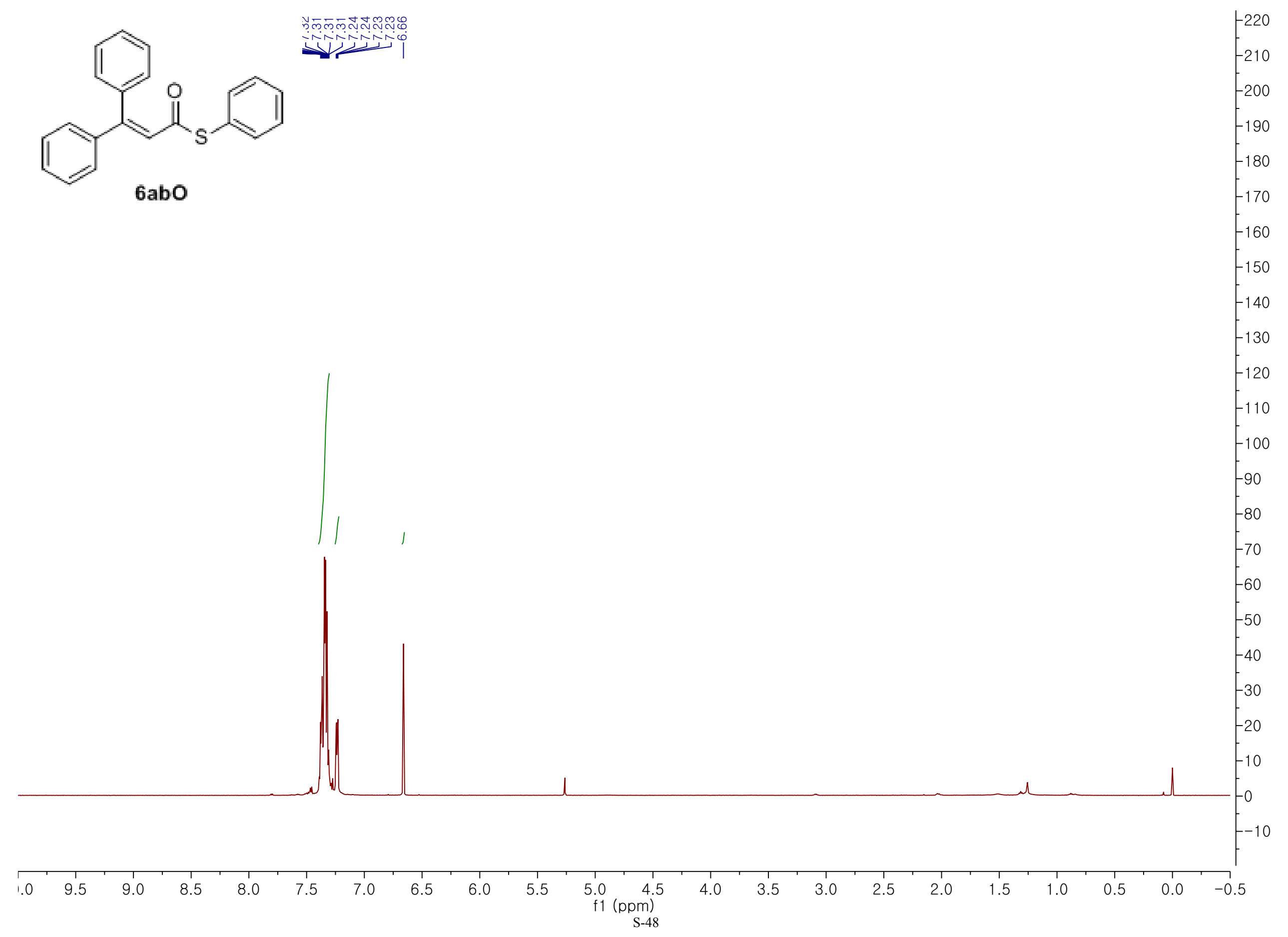




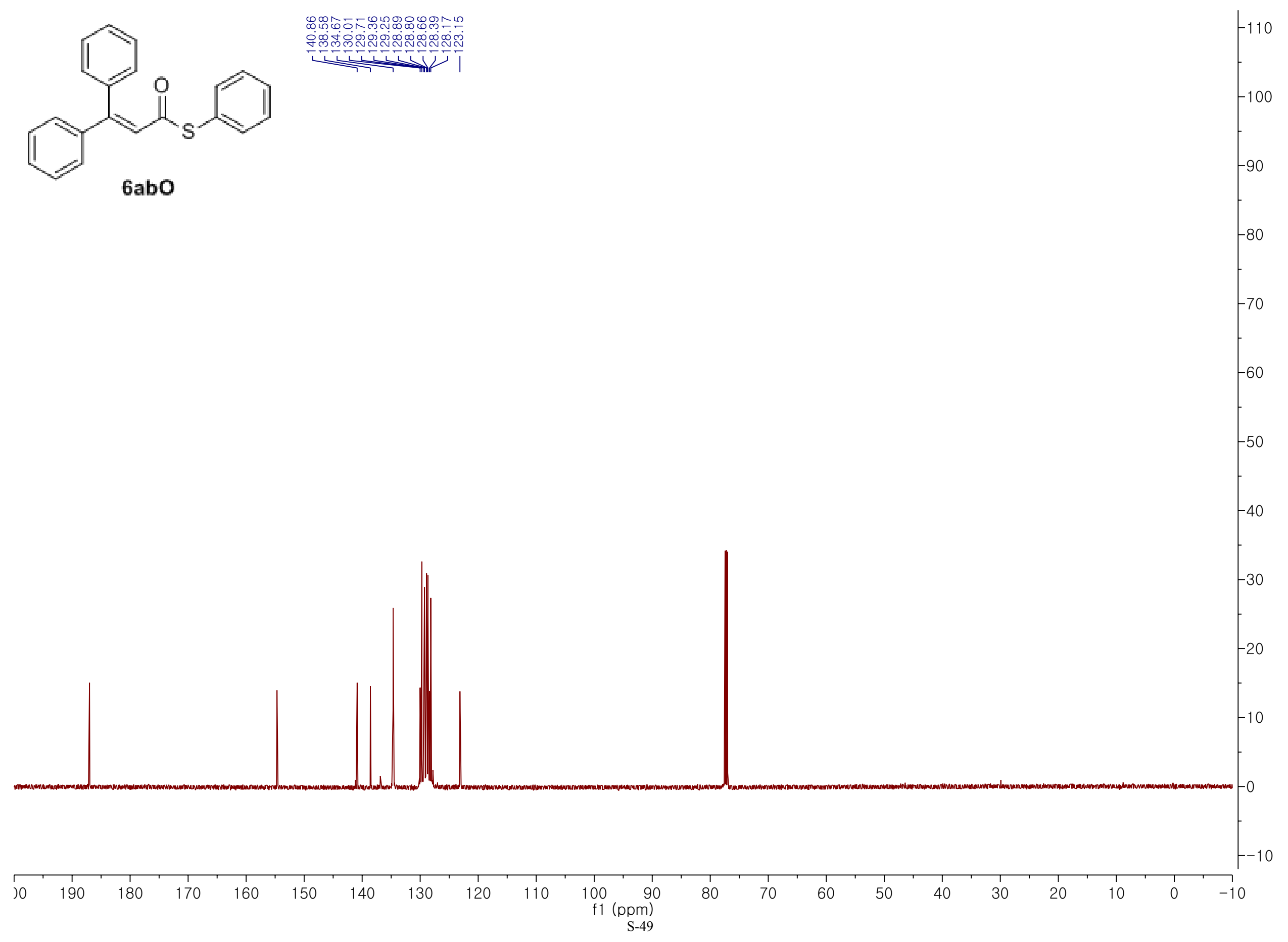




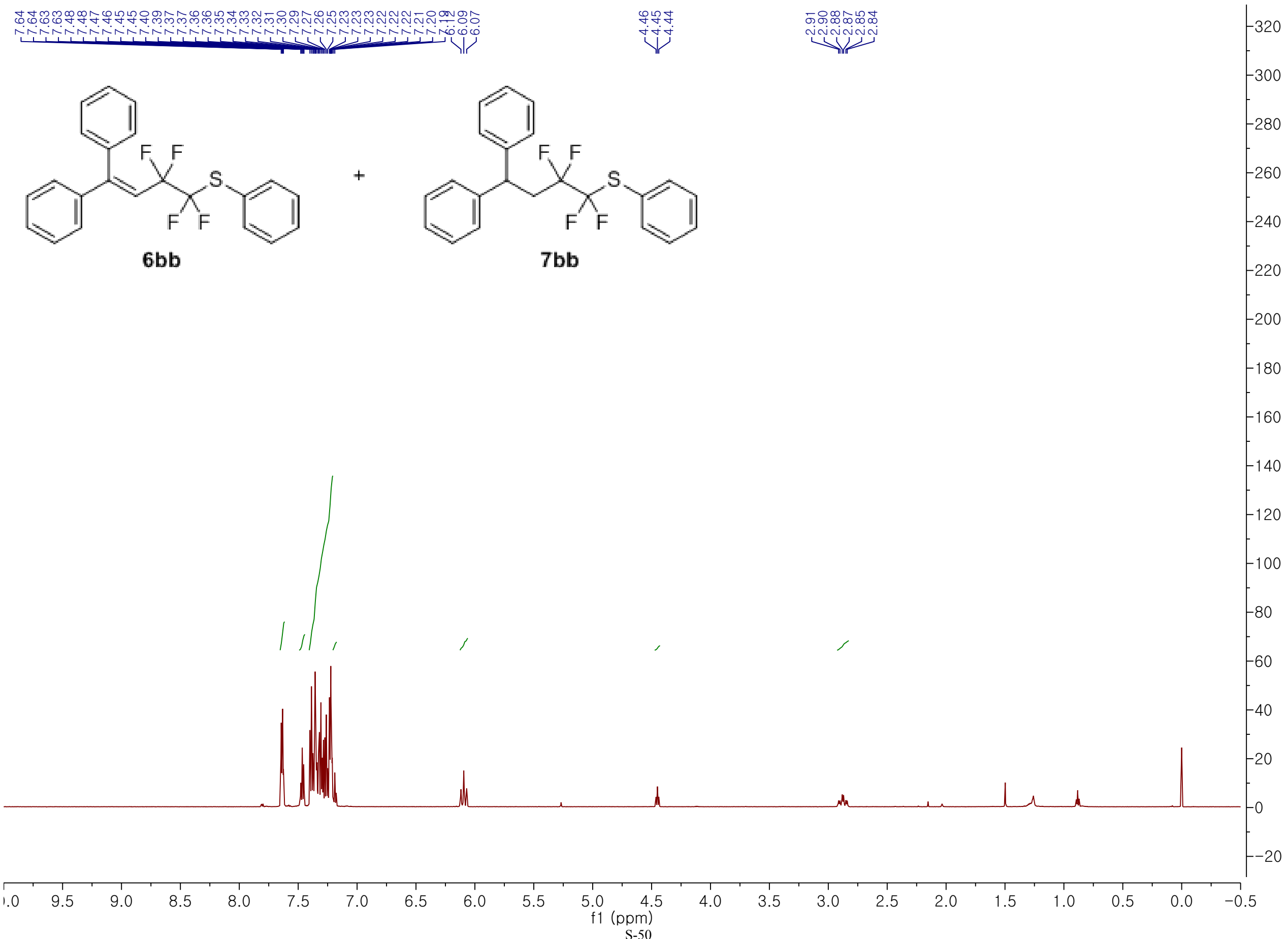




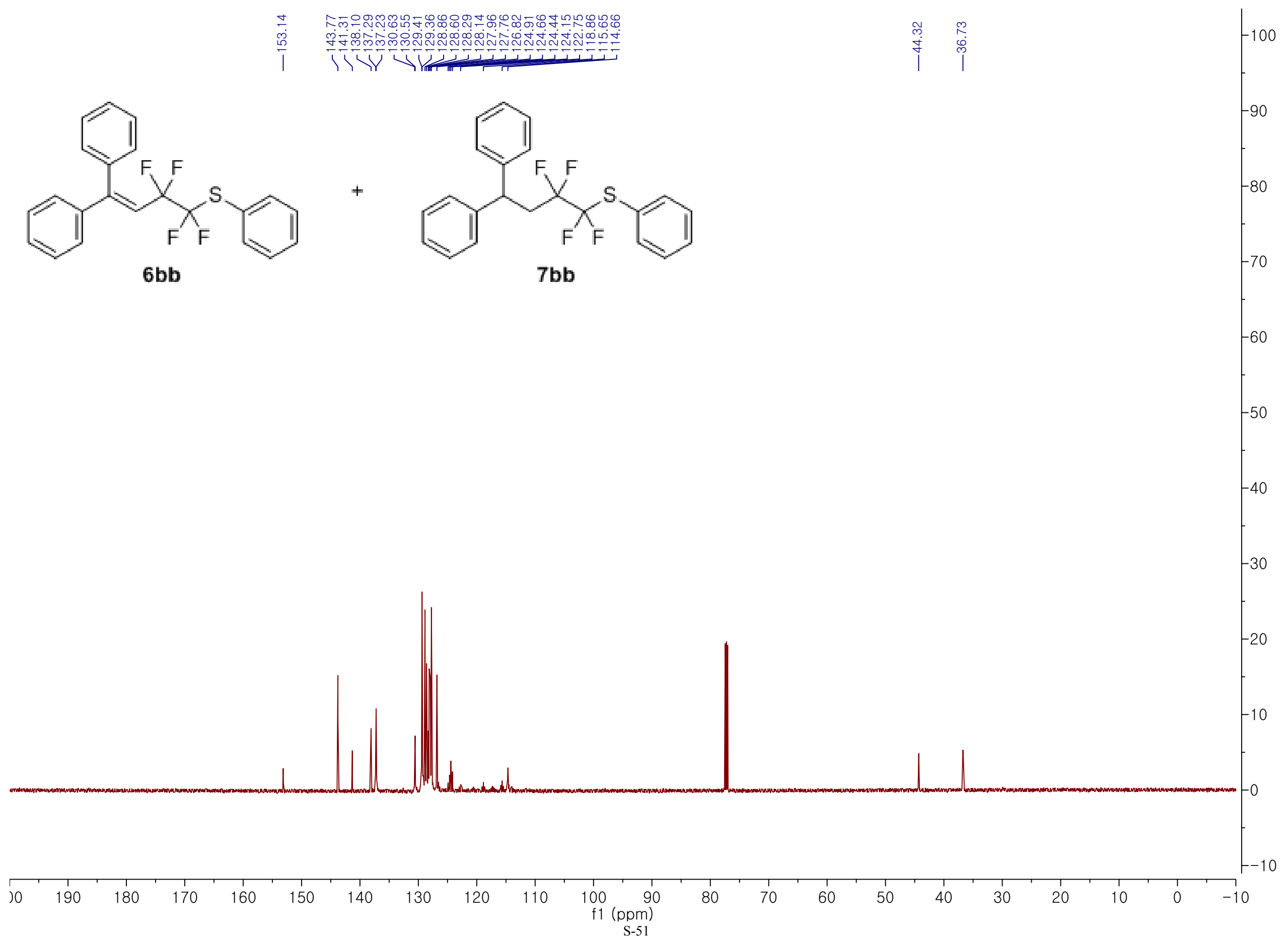




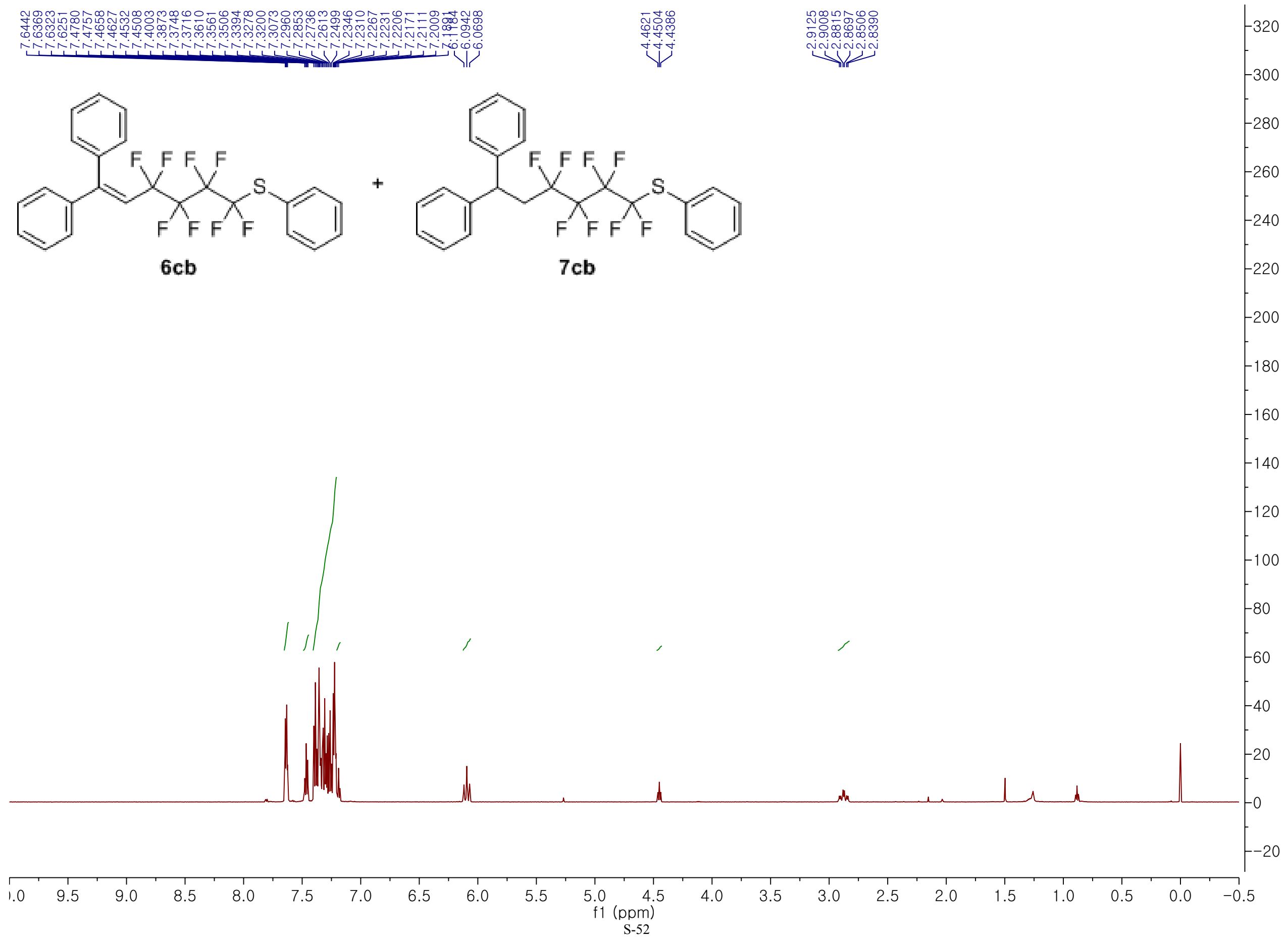




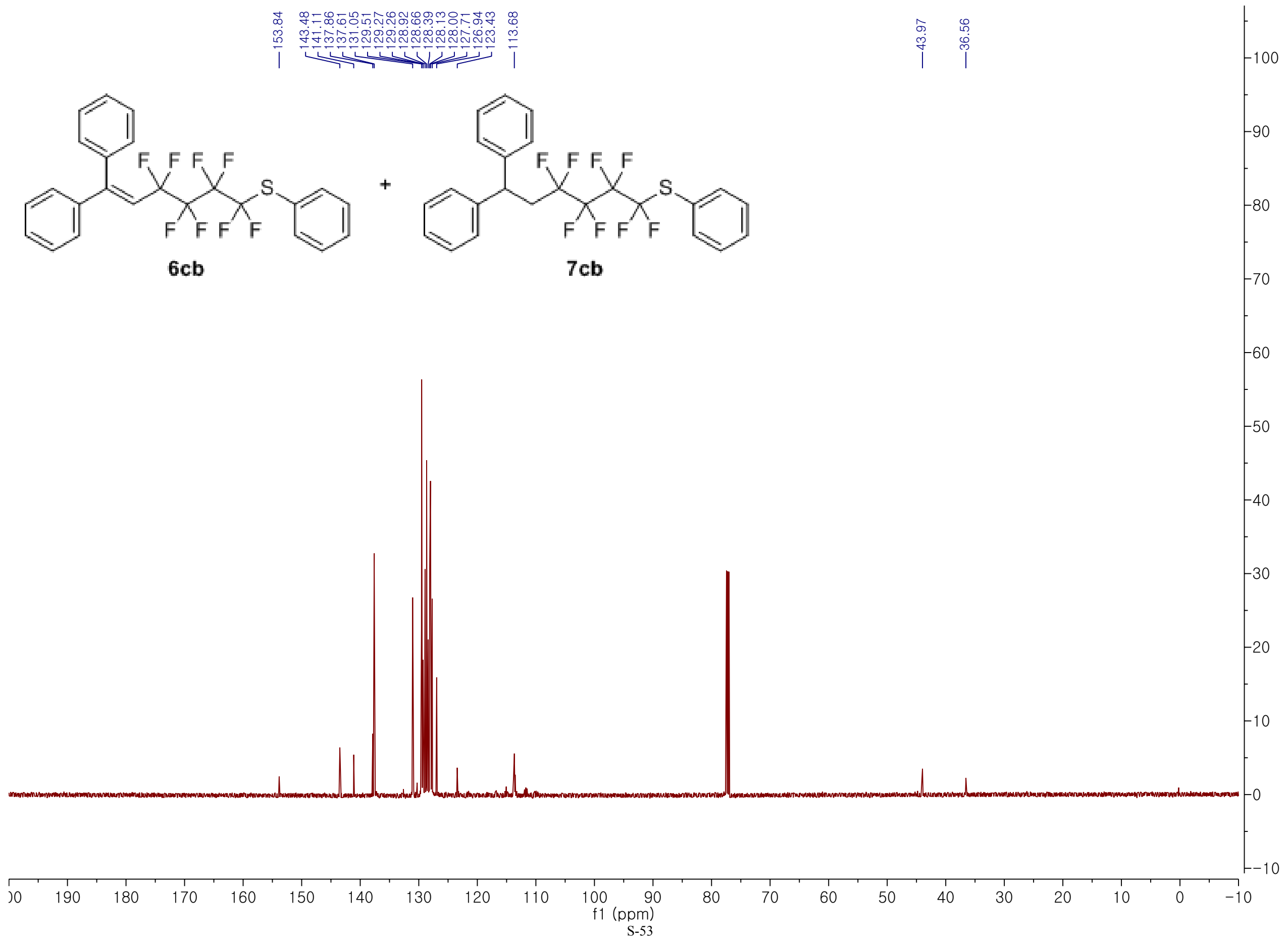




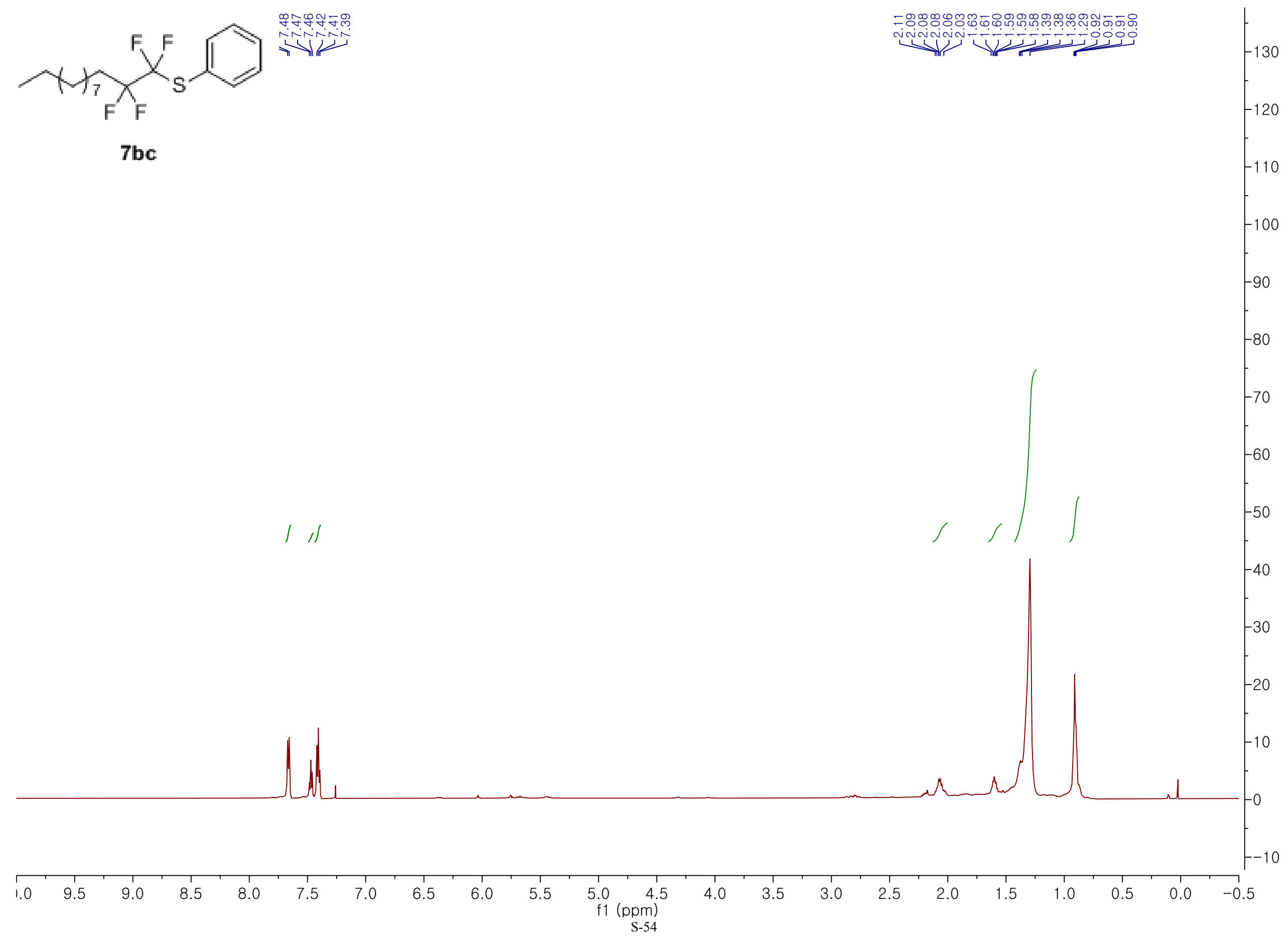




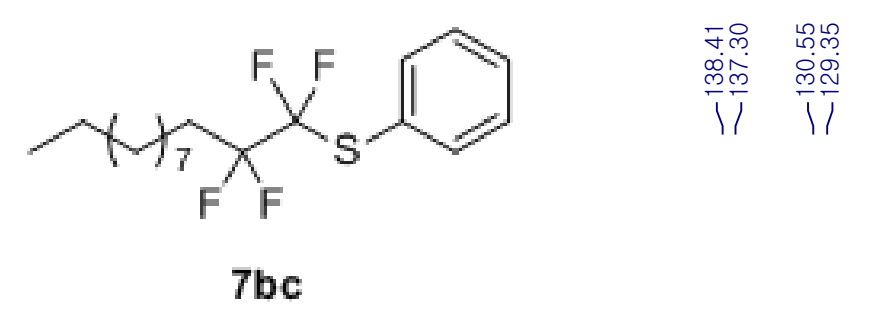

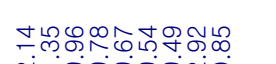

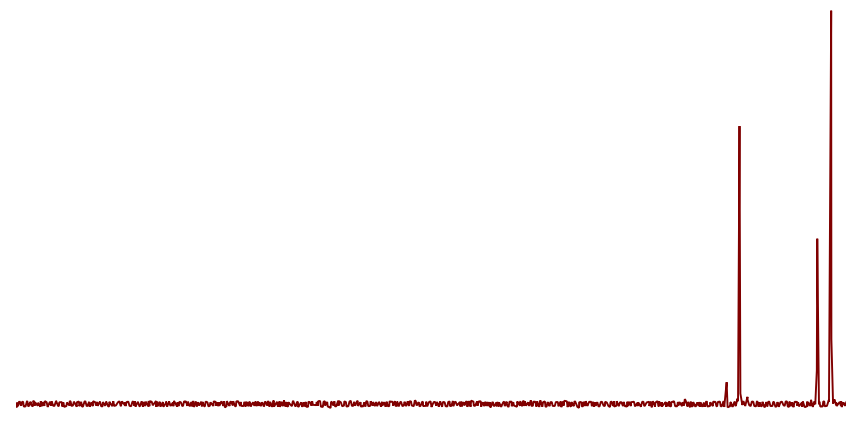




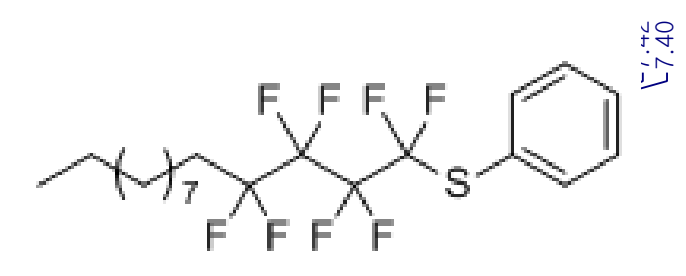

$7 \mathrm{cc}$

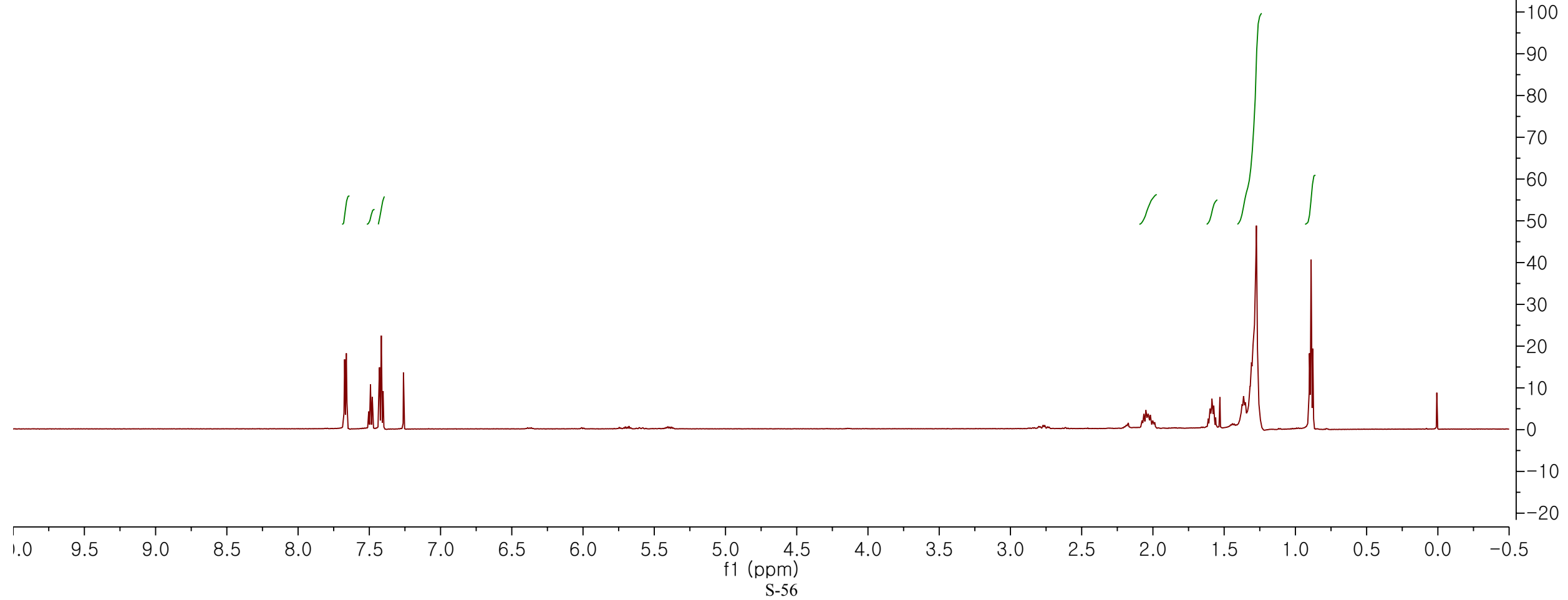




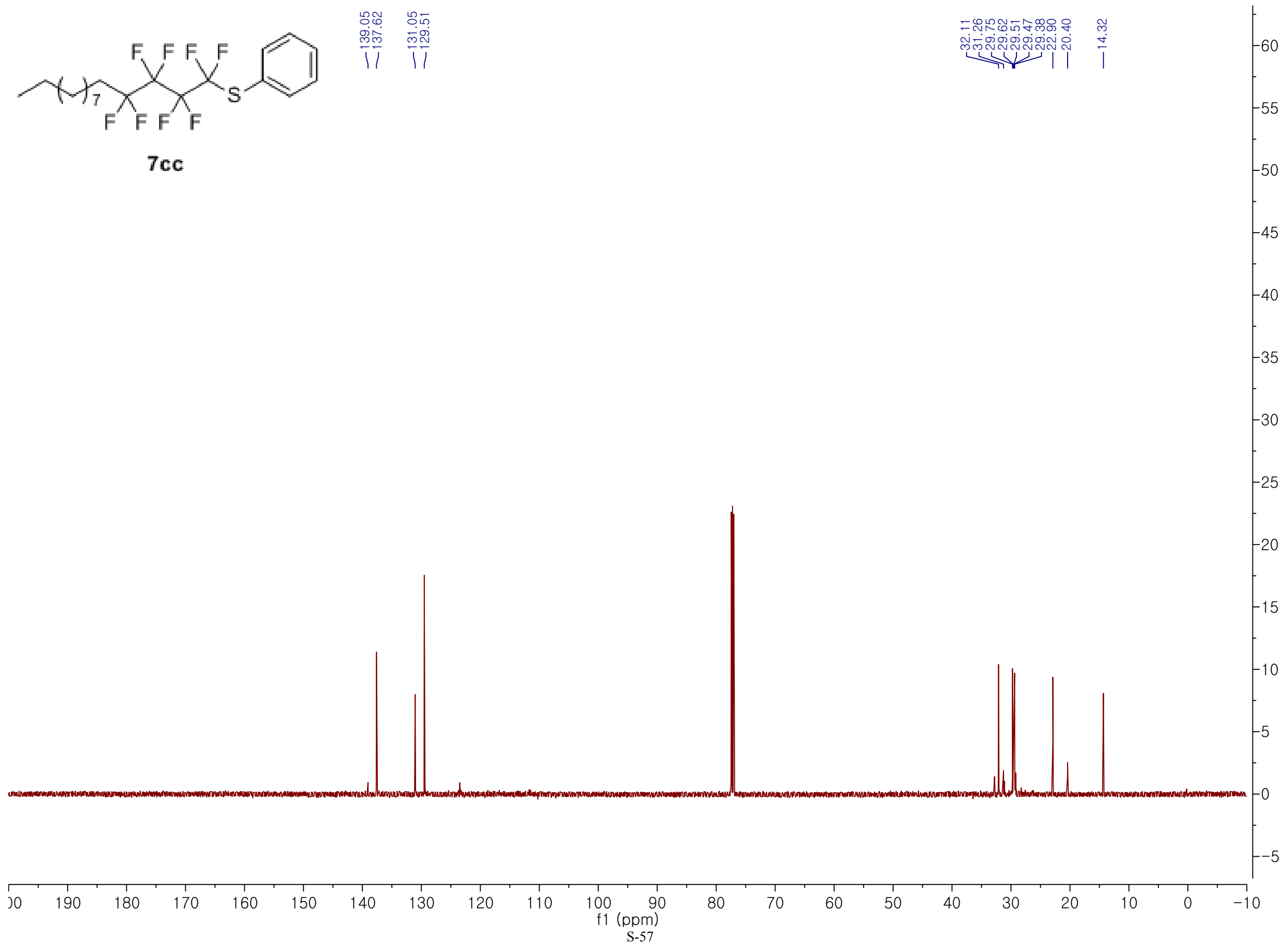




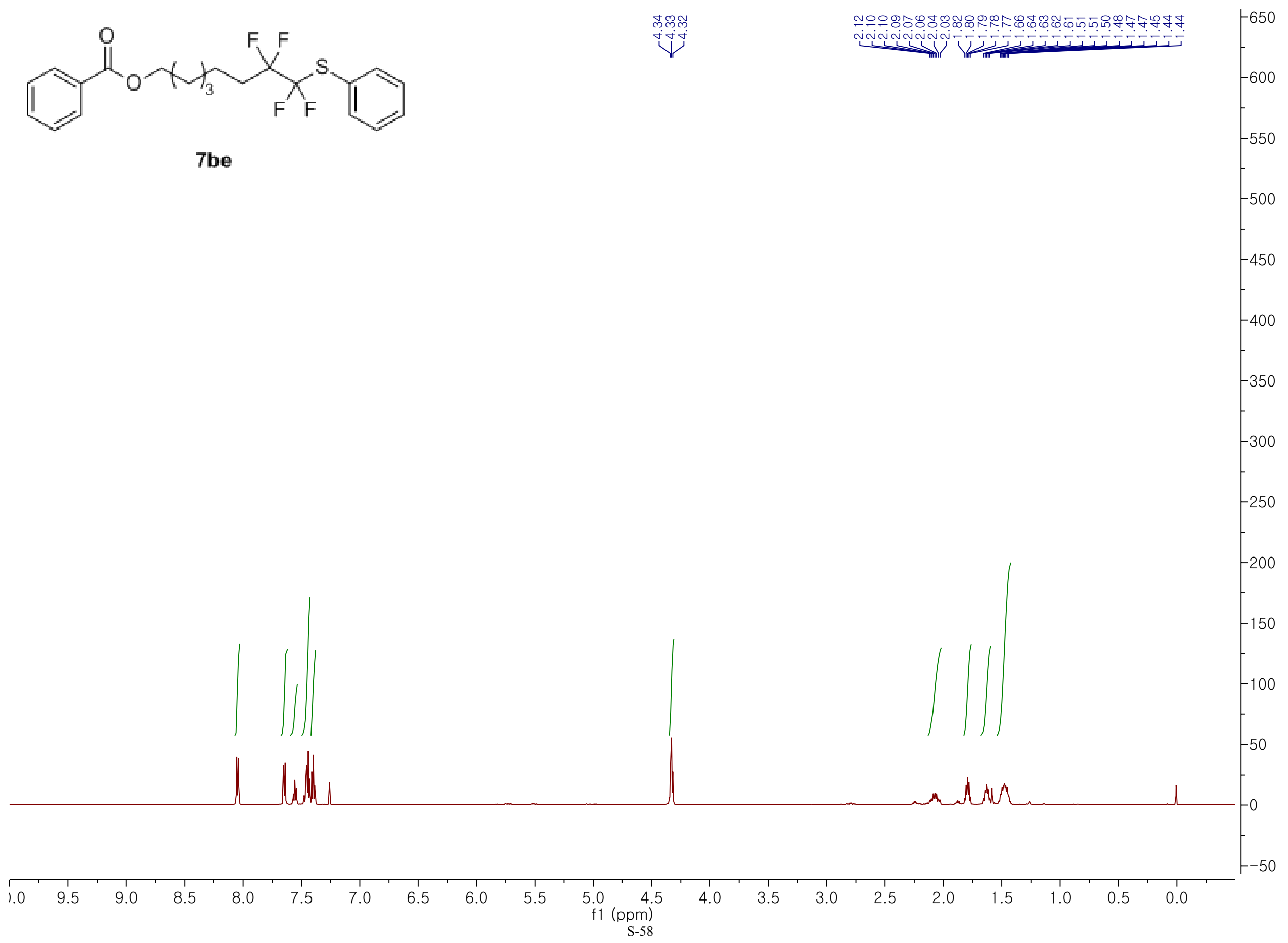




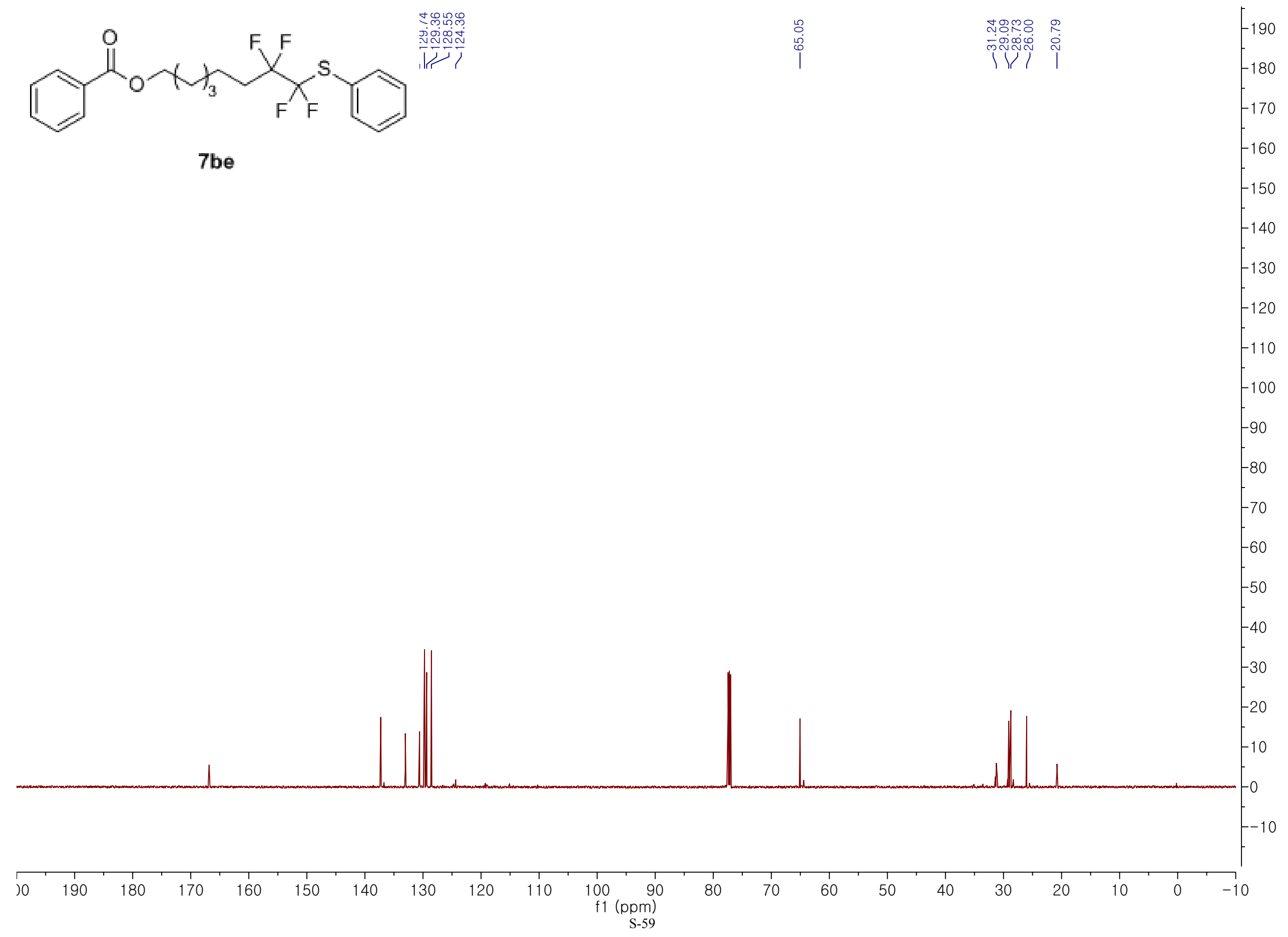




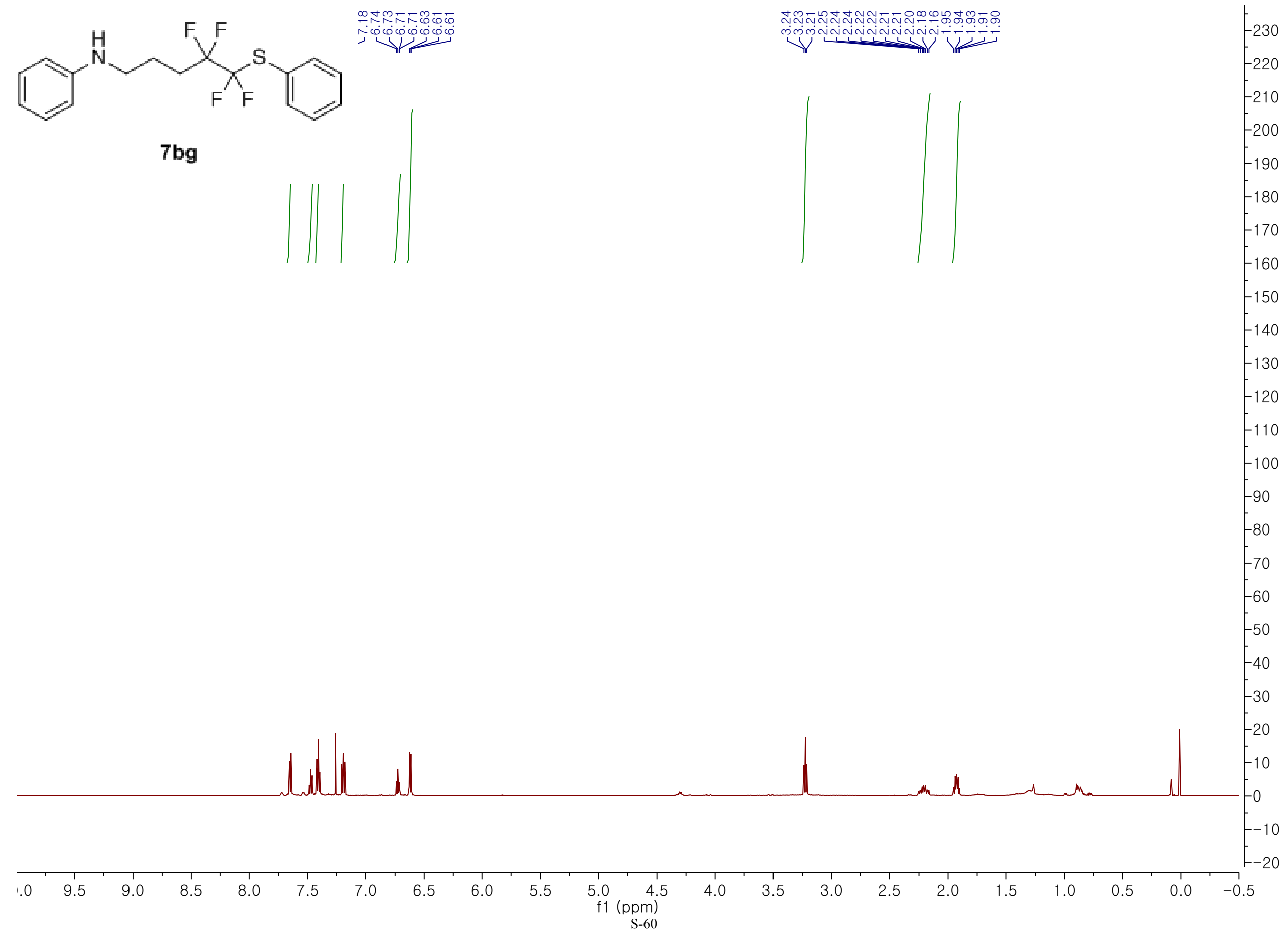




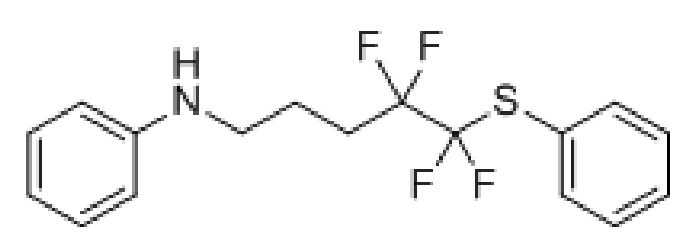

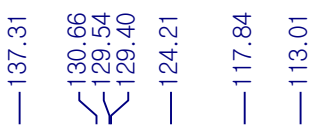

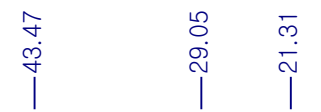

7 bg

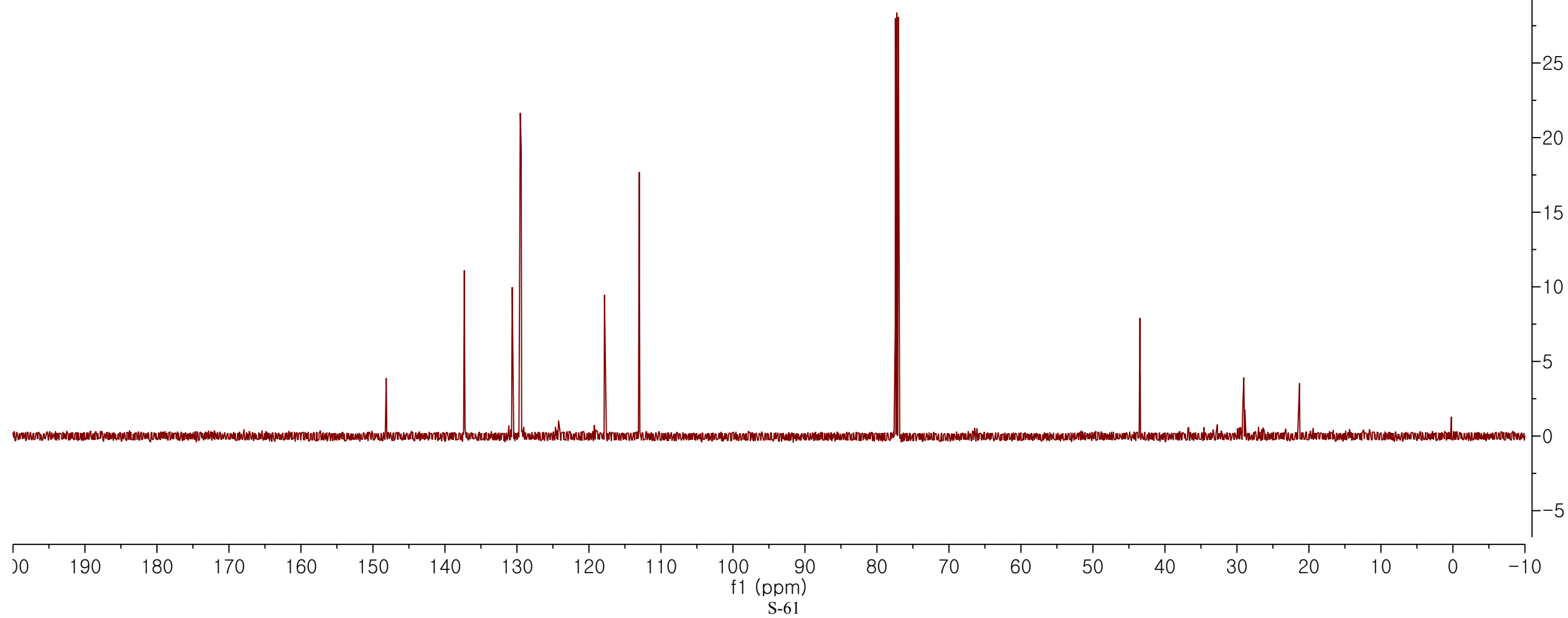




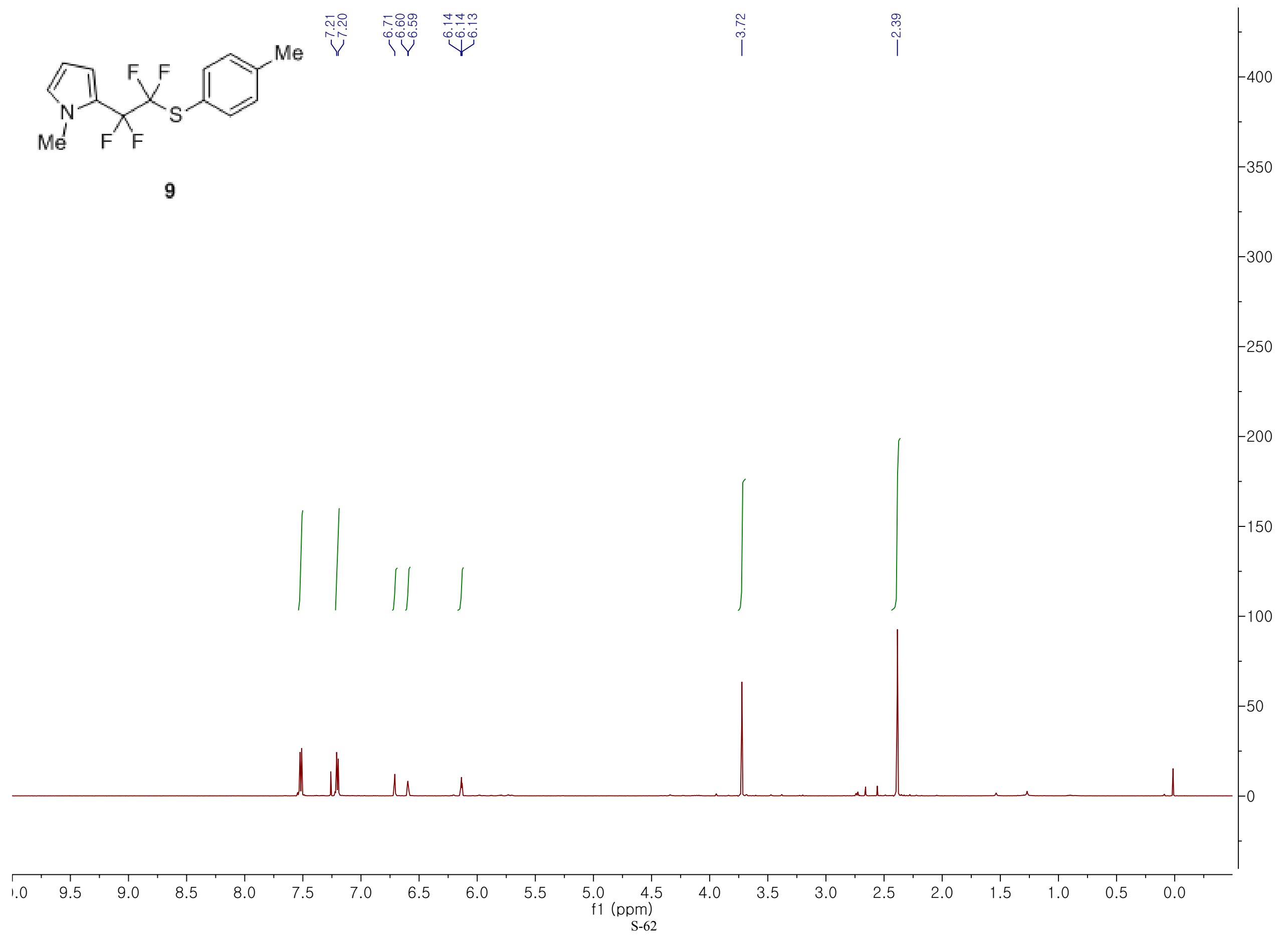




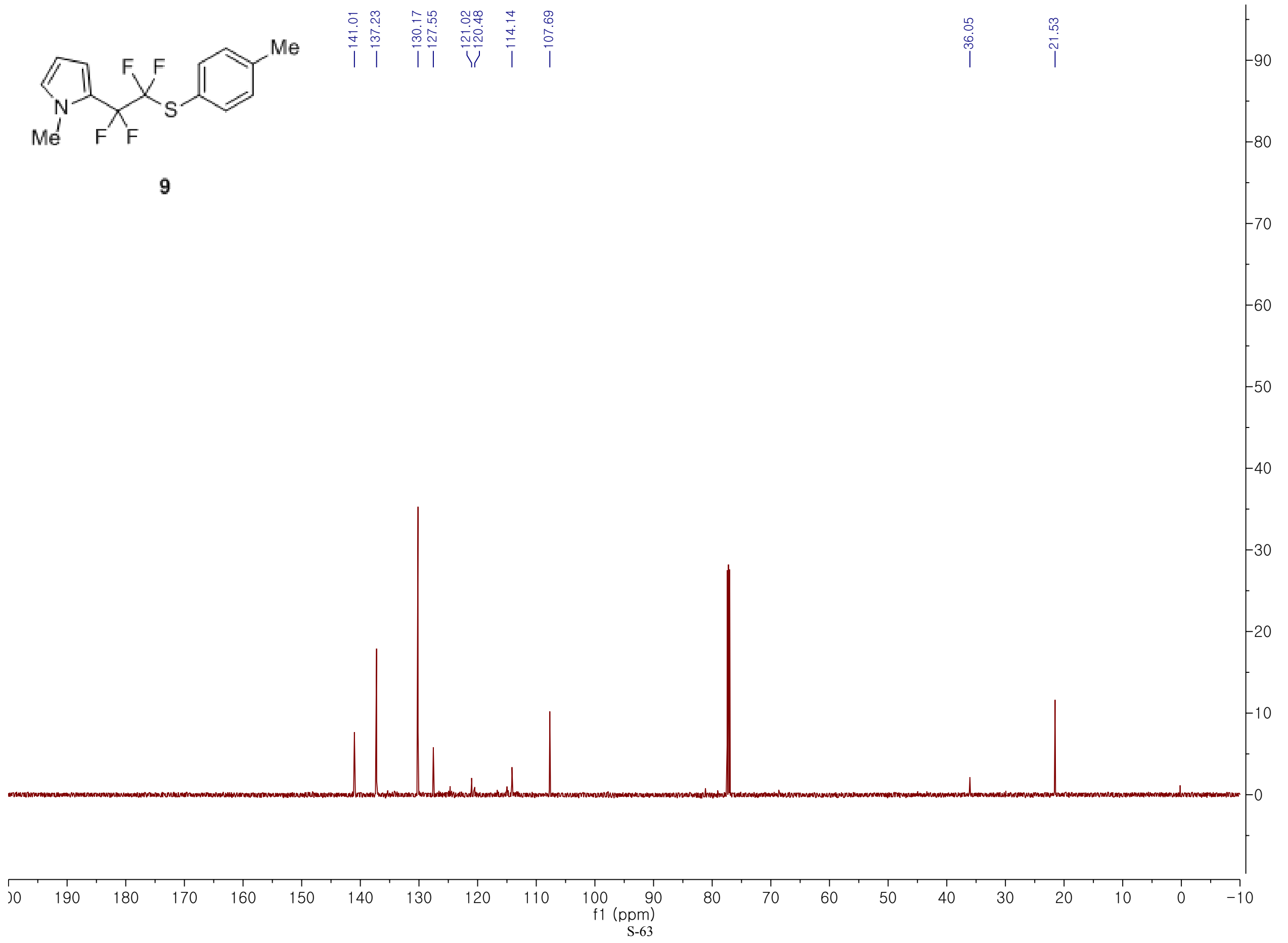




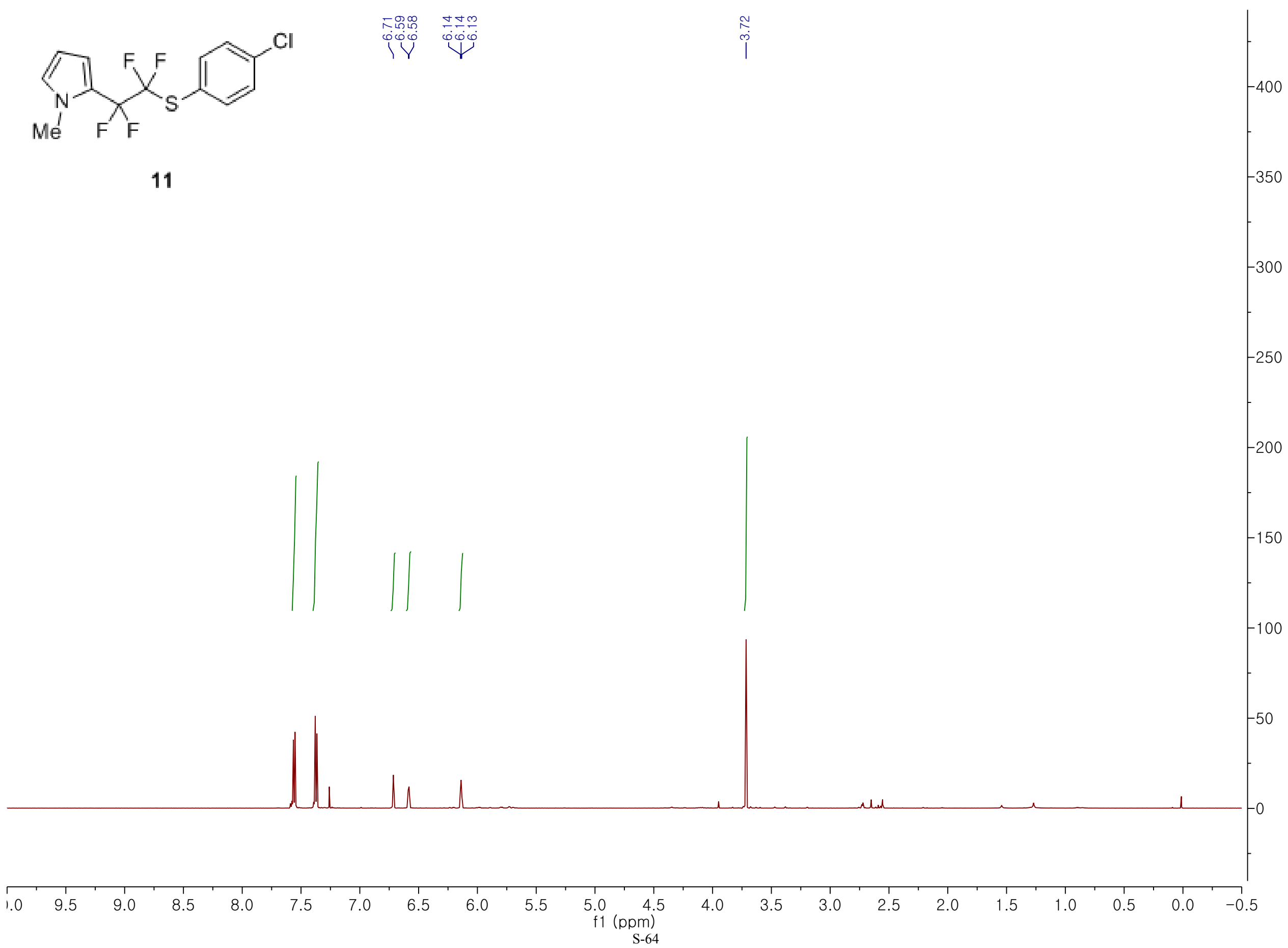




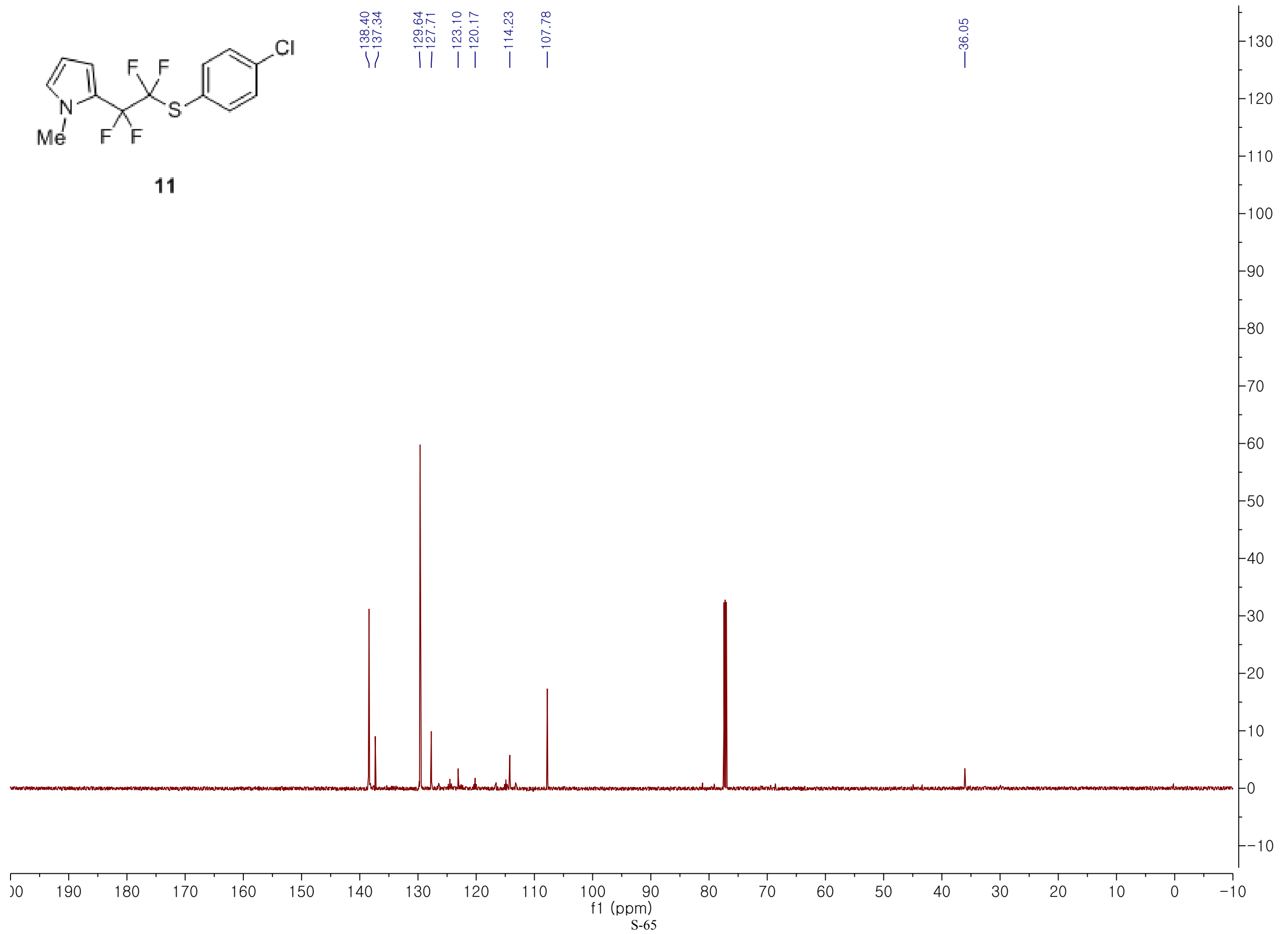




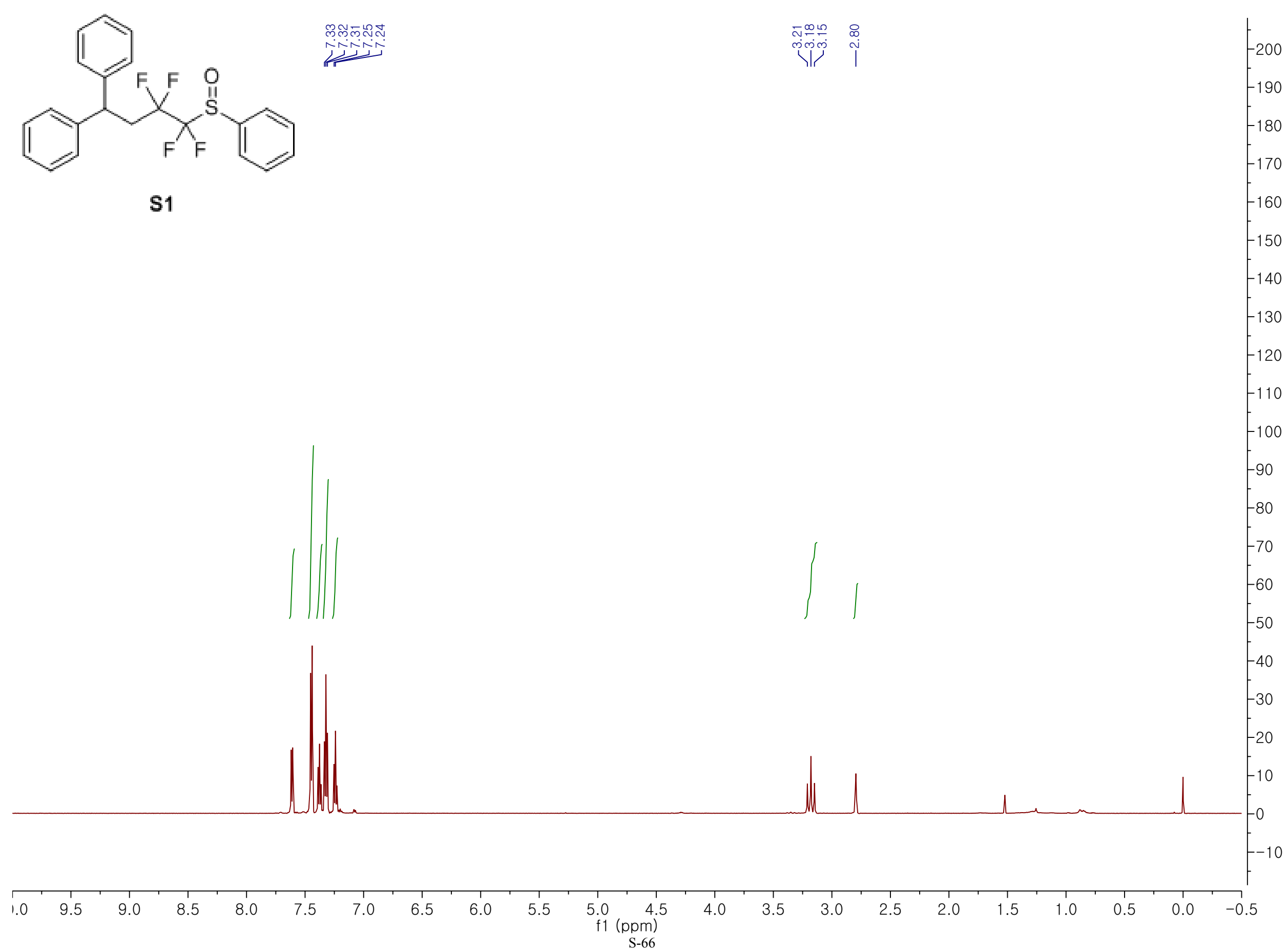




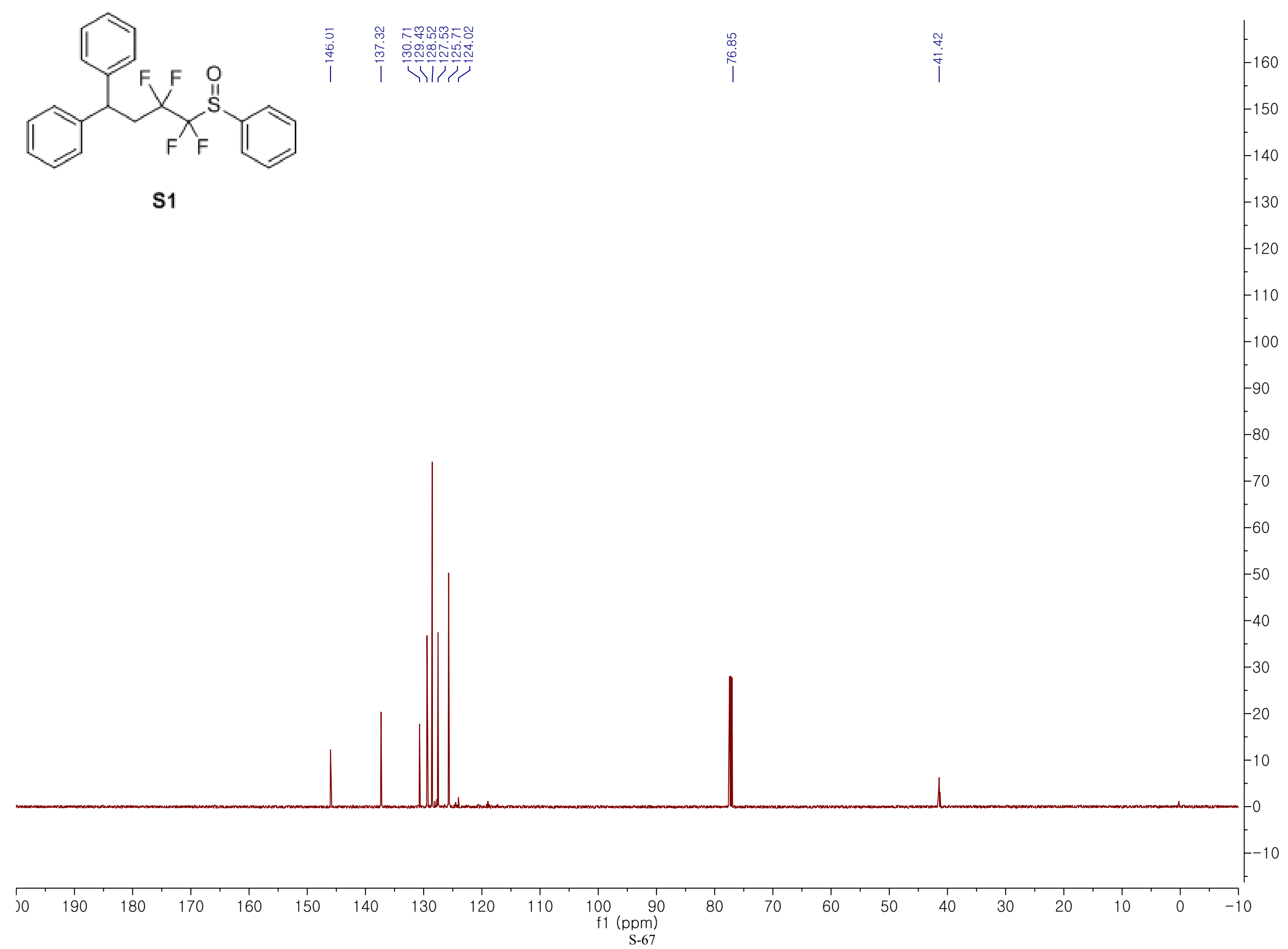

